\title{
Linear Array Ultrasonic Testing of a Thick Concrete Specimen for Nondestructive Evaluation
}

Dwight Clayton N. Dianne Bull Ezell Lev Khazanovich Mattia Zammerachi

April 2017 


\title{
DOCUMENT AVAILABILITY
}

Reports produced after January 1, 1996, are generally available free via US Department of Energy (DOE) SciTech Connect.

Website http://www.osti.gov/scitech/

Reports produced before January 1, 1996, may be purchased by members of the public from the following source:

\author{
National Technical Information Service \\ 5285 Port Royal Road \\ Springfield, VA 22161 \\ Telephone 703-605-6000 (1-800-553-6847) \\ TDD 703-487-4639 \\ Fax 703-605-6900 \\ E-mail info@ntis.gov \\ Website http://classic.ntis.gov/
}

Reports are available to DOE employees, DOE contractors, Energy Technology Data Exchange representatives, and International Nuclear Information System representatives from the following source:

Office of Scientific and Technical Information

PO Box 62

Oak Ridge, TN 37831

Telephone 865-576-8401

Fax 865-576-5728

E-mail reports@osti.gov

Website http://www.osti.gov/contact.html

This report was prepared as an account of work sponsored by an agency of the United States Government. Neither the United States Government nor any agency thereof, nor any of their employees, makes any warranty, express or implied, or assumes any legal liability or responsibility for the accuracy, completeness, or usefulness of any information, apparatus, product, or process disclosed, or represents that its use would not infringe privately owned rights. Reference herein to any specific commercial product, process, or service by trade name, trademark, manufacturer, or otherwise, does not necessarily constitute or imply its endorsement, recommendation, or favoring by the United States Government or any agency thereof. The views and opinions of authors expressed herein do not necessarily state or reflect those of the United States Government or any agency thereof. 


\title{
LINEAR ARRAY ULTRASONIC TESTING OF A THICK CONCRETE SPECIMEN FOR NONDESTRUCTIVE EVALUATION
}

\author{
Dwight Clayton, Oak Ridge National Laboratory \\ N. Dianne Bull Ezell, Oak Ridge National Laboratory \\ Lev Khazanovich, University of Minnesota \\ Mattia Zammerachi, University of Minnesota
}

Date Published: April 2017

\author{
Prepared by \\ OAK RIDGE NATIONAL LABORATORY \\ Oak Ridge, TN 37831-6283 \\ managed by \\ UT-BATTELLE, LLC \\ for the \\ US DEPARTMENT OF ENERGY \\ under contract DE-AC05-00OR22725
}





\section{CONTENTS}

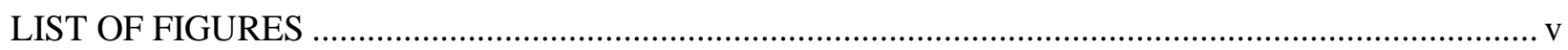

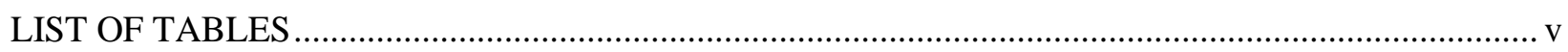

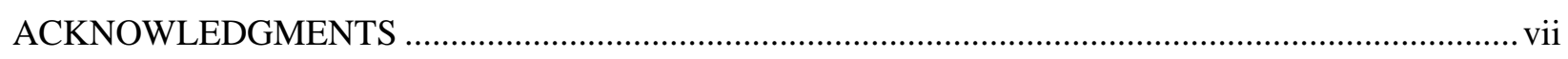

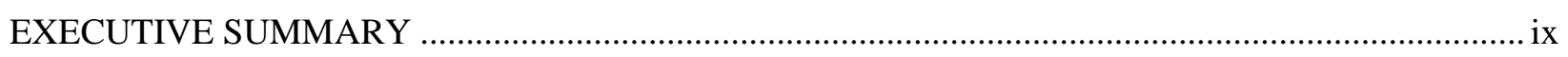

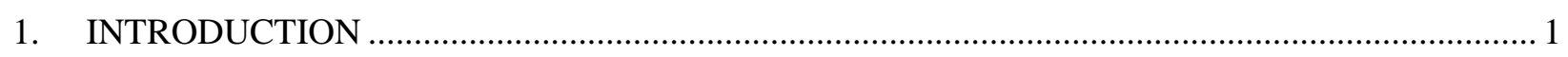

2. SPECIMEN DIMENSIONS, REINFORCEMENT, AND DEFECTS ............................................ 1

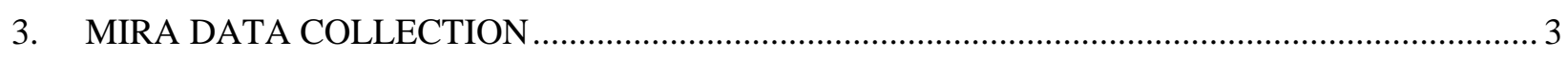

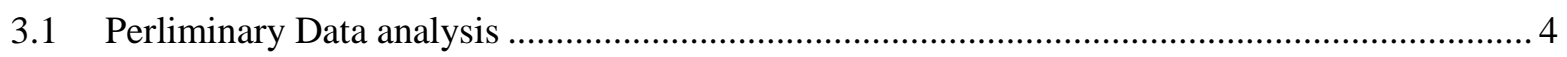

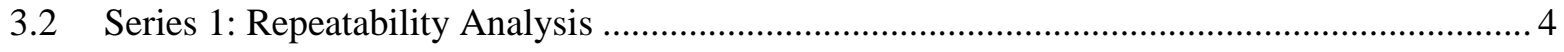

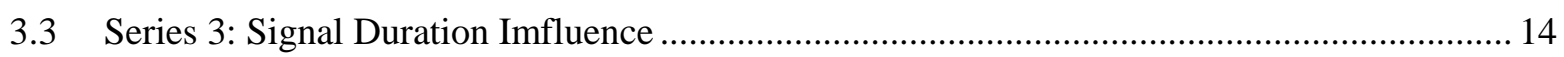

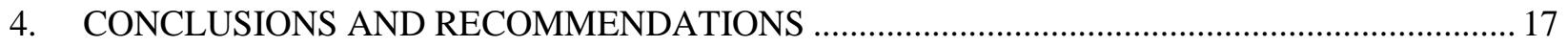

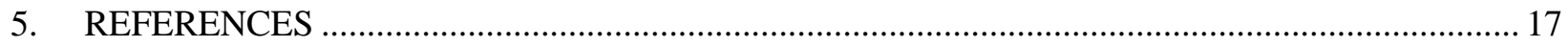

APPENDIX A. FILE NAMES AND TEST PROGRAM ……........................................................ A-1

APPENDIX B. PANORAMIC IMAGES FOR HEIGHT ORIENTATION ….................................... B-1

APPENDIX C. PANORAMIC IMAGES FOR LENGTH ORIENTATION .......................................... C-1 



\section{LIST OF FIGURES}

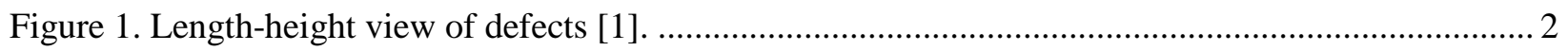

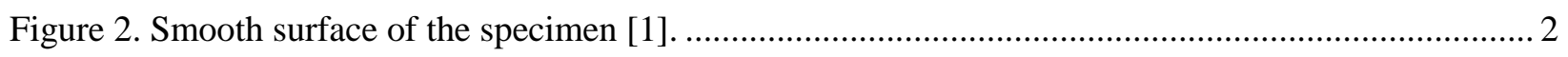

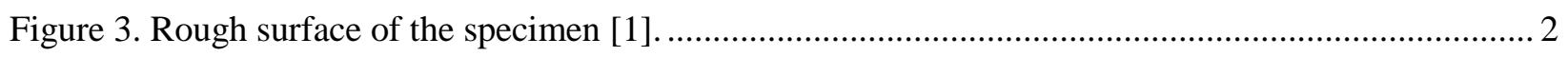

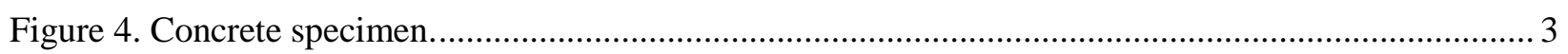

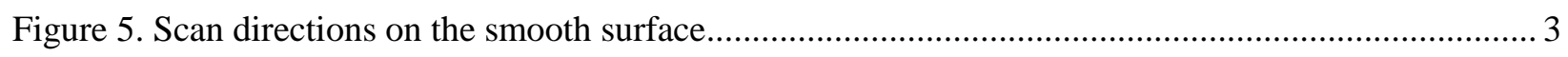

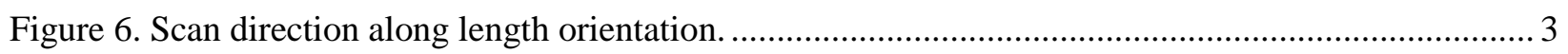

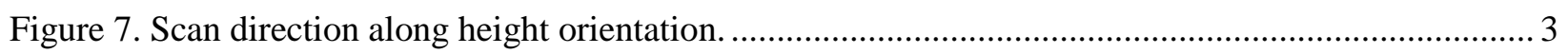

Figure 8. Mean surface velocities from testing with and without lifting the device................................. 6

Figure 9. Mean Hilbert transform indicators (HTI) from testing with and without lifting the device.......... 7

Figure 10. SAFT-IA reconstructions for location 4, row 9: (a) without lifting the device and (b)

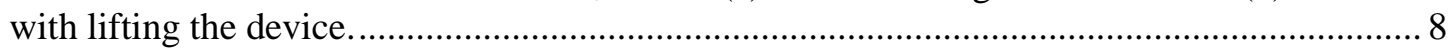

Figure 11. SAFT-IA reconstructions for location 7, row 9: (a) without lifting the device and (b)

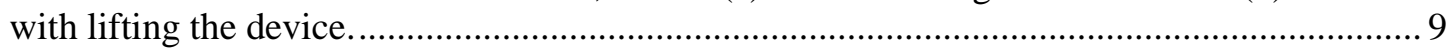

Figure 12. SAFT-IA reconstructions for location 14, row 9 (a) without lifting the device and (b)

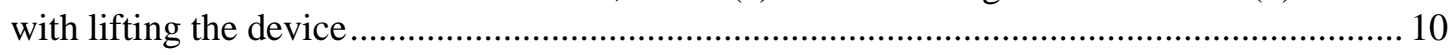

Figure 13. Comparison of panoramic reconstructions obtained in this study (a) with those reported

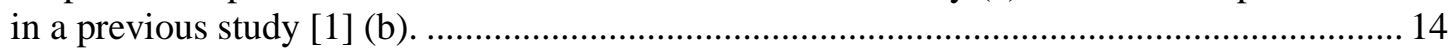

Figure 14. Location of the D13 defect and direction of the panoramic B-scan, rows 8 and 9, length direction: (a) D13 length by depth NL, (b) D13 length by height NL, and (c) D13

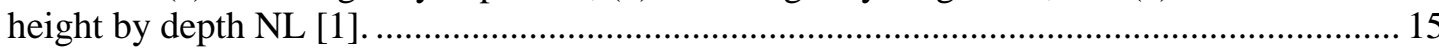

Figure 15. Panoramic reconstructions for row 8 with different impulse durations: (a) 1 half-period, (b) 2 half-periods, (c) 4 half-periods, and (d) 16 half-periods. 16

\section{LIST OF TABLES}

Table 1. Statistical summary of computed surface velocities for each location and test method 5

Table 2. Statistical summary of the computed Hilbert transform indicators (HTIs) for each location

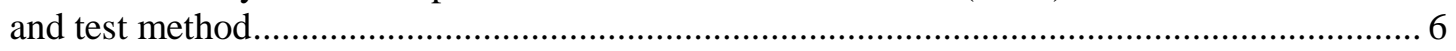

Table 3. Pearson correlation coefficients for $50 \mathrm{kHz}$ without lifting .................................................. 12

Table 4. Pearson correlation coefficients for $50 \mathrm{kHz}$ with lifting........................................................ 12

Table 5. Pearson correlation coefficients for $30 \mathrm{kHz}$ without lifting .................................................... 13

Table 6. Pearson correlation coefficients for $100 \mathrm{kHz}$ without lifting .................................................. 13 



\section{ACKNOWLEDGMENTS}

This work is funded by the US Department of Energy Office of Nuclear Energy under the Light Water Reactor Sustainability program. 



\section{EXECUTIVE SUMMARY}

The University of Minnesota and Oak Ridge National Laboratory are collaborating on the design and construction of a concrete specimen with sufficient reinforcement density and cross-sectional size to represent a light water reactor containment wall with various defects. The preliminary analysis of the collected data using an extended synthetic aperture focusing technique (SAFT) reconstruction algorithm indicated the ultrasound array technology had great potential for locating relatively shallow stresses. However, the resolution and reliability of the analysis is inversely proportional to the defect depth and the amount of reinforcement between the measurement point and the defect location. The objective of this round of testing was to evaluate repeatability of the obtained reconstructions from measurements with different frequencies and to examine the effect of the duration of the excitation ultrasound signal on the resulting reconstructions.

Two series of tests were performed in this study. The objective of the first series was to evaluate repeatability of the measurements and resulting reconstructed images. The measurements use three center frequencies. Five measurements were performed at each location with and without lifting the device. The analysis of the collected data suggested that a linear array ultrasound system could produce reliably repeatable reconstructions using $50 \mathrm{kHz}$ signals for relatively shallow depths (less than $0.5 \mathrm{~m}$ ). However, for reconstructions at greater depths the use of lower frequency and/or signal filtering to reduce the effect of signal noise may be required.

The objective of the second series of testing was to obtain measurements with various impulse signal durations. The entire grid on the smooth surface was tested various impulse signals at four different durations. An analysis of the resulting extended SAFT reconstructions suggested that Kirchhoff-based migration leads to easier to interpret reconstructions when shorter duration impulse signals are used. Longer duration impulses may provide useful information for model-based reconstructions. 



\section{INTRODUCTION}

Portland cement concrete (PCC)-based structures are typically used in light water reactor (LWR) plants to provide the foundation, support, shielding, and containment functions. Extending the life of the existing LWR fleet to 60 years and beyond will likely increase the susceptibility to and severity of known forms of degradation. Degradation of PCC and other defects are significant threats to the structural integrity and safe operation of aging LWRs. Moreover, the nondestructive evaluation (NDE) of LWR reinforced concrete members involves unique complications. The presence of reinforcement and aggregates, coupled with access limitations (i.e., one-sided access), imposes additional challenges when attempting to detect damage at depths of $1 \mathrm{~m}$ or more. Thus, an in situ and objective method for nondestructively detecting subsurface damage has yet to be successfully developed and implemented.

In previous studies, the University of Minnesota and Oak Ridge National Laboratory collaborated on the design and construction of a concrete specimen with sufficient reinforcement density and cross-sectional size to represent an LWR containment wall with various defects [1]. A database of the ultrasound measurements was developed using a linear array system with dry-point contact shear wave ultrasonic transducers. The preliminary analyses of the collected data using an extended synthetic aperture focusing technique (SAFT) reconstruction indicated the ultrasound array technology had great potential for locating relatively shallow stresses. However, the resolution and reliability of the analyses were inversely proportional to the defect depth and the amount of reinforcement between the measurement point and the defect location. The objective of this round of testing was to evaluate repeatability of the obtained reconstructions from measurements with different frequencies and to evaluate the effect of the duration of the ultrasound signal on the resulting reconstructions.

\section{SPECIMEN DIMENSIONS, REINFORCEMENT, AND DEFECTS}

The thick specimen was constructed in the Multi-Axial Subassemblage Testing (MAST) System Laboratory at the University of Minnesota in the spring of 2015. It is a $2.134 \mathrm{~m} \times 2.134 \mathrm{~m} \times 1.016 \mathrm{~m}$ concrete test specimen with 20 deliberately embedded defects. It contains \#18 rebars at 12 in. spacing in both horizontal and vertical orientations. This provides a realistic reinforcement size that also allows for space between reinforcements for semi-controlled evaluation of the effects of concrete depth on defect characterization. This also allows for differentiation between complexities caused by dense levels of reinforcement versus those caused by depth of penetration within concrete while still observing the effects of the uniquely large diameter reinforcement used in LWR structures.

Simulated defects were embedded to determine how the current state-of-the-practice NDE techniques would be able to determine various forms of degradation in nuclear power plant (NPP) concrete structures. The constructed specimen was designed to allow for assessment of controlled benchmark defects, from previous studies, in a more heavily reinforced concrete structure and evaluation of realistic defects to ensure that the correct type of features for effective NPP NDE were included [1]. Figure 1

presents a schematic of the specimen cross-sectional design with locations of various defects. Full details on the geometry, location, and nature of the defects can be found in previous documentation [1]. 


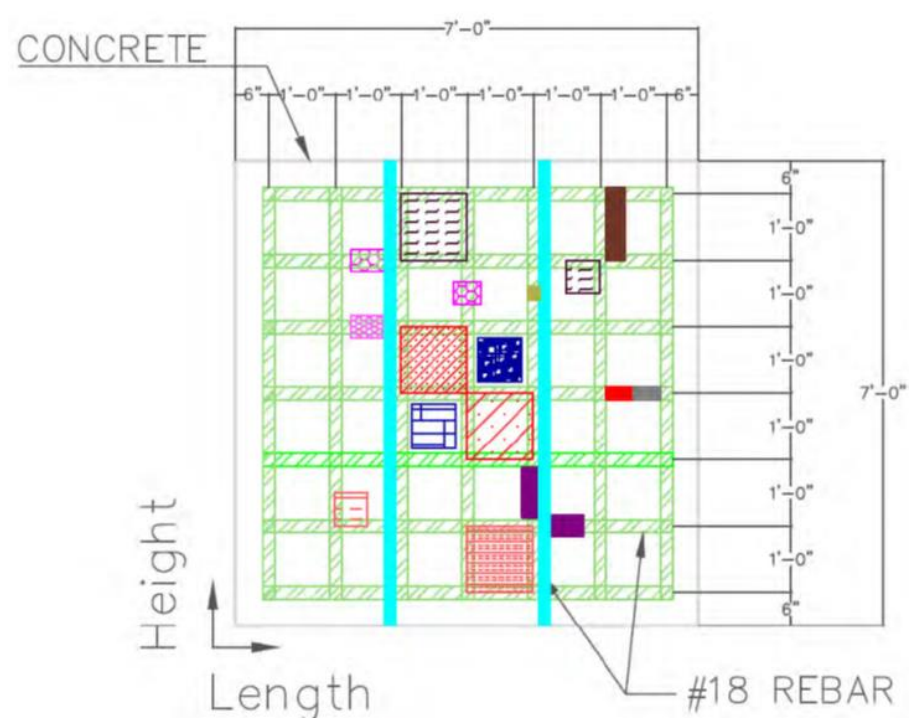

Figure 1. Length-height view of defects [1].

The first round of ultrasound testing was conducted at the University of Minnesota MAST Lab in 2015. At that time the specimen was placed in vertical position and two large surfaces of the specimen were gridded in 4 in. $(102 \mathrm{~mm})$ squares covering in each direction the 84 in. by 84 in. (see Figure 2 and Figure $3)$.

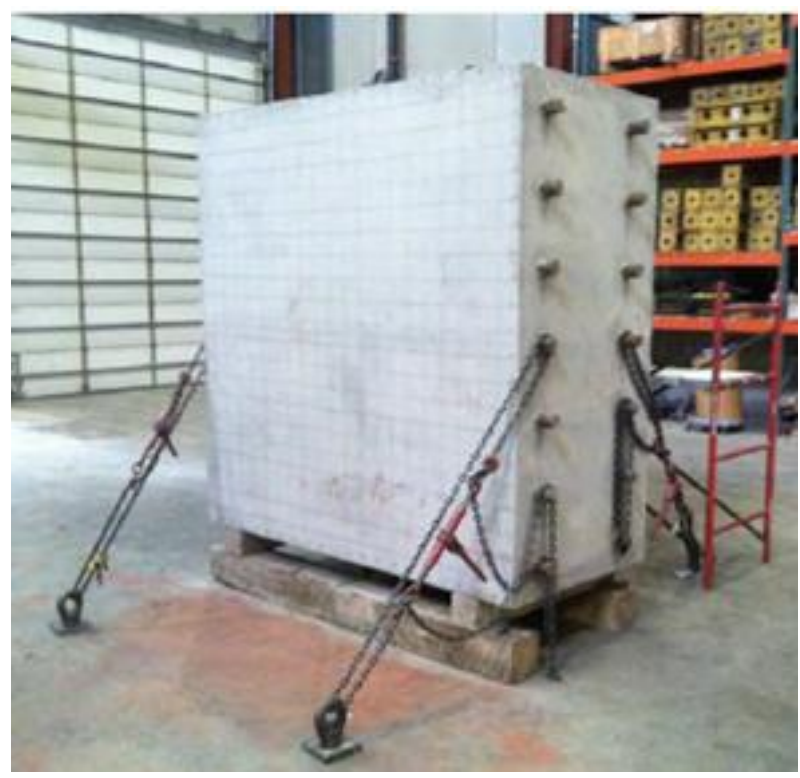

Figure 2. Smooth surface of the specimen [1].

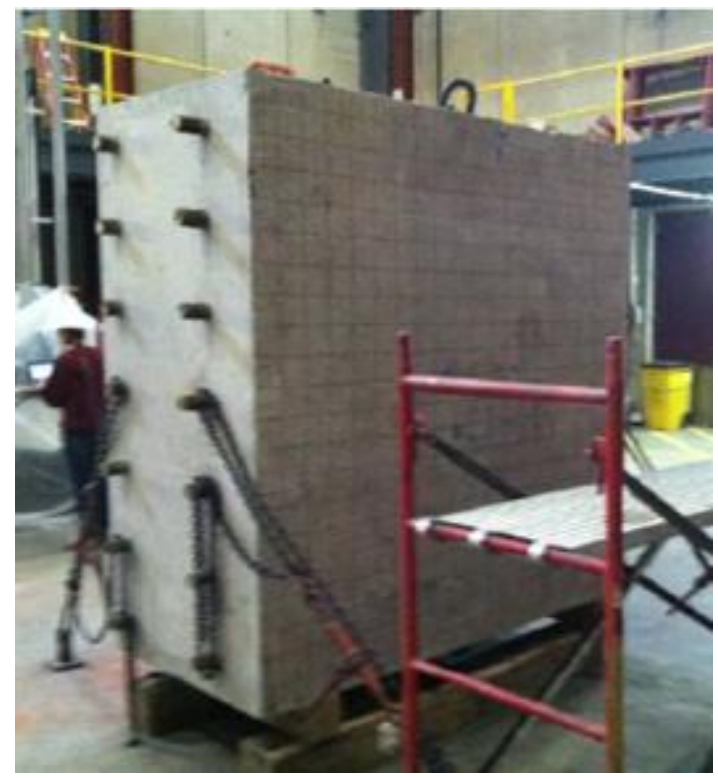

Figure 3. Rough surface of the specimen [1].

In the fall of 2015 the specimen was moved to the Minnesota Road Test Facility located in Monticello, Minnesota. Currently it is stored in a horizontal position (Figure 4), so only one large side of the specimen, referred to as "smooth side," was available for testing in the previous study [1]. However, to be consistent with the previous study, the directions of testing will be referred to as along height and length (see Figure 5). The grid from the original study was reused in the current testing. The rows and columns are labeled from 1 to 19 (Figure 6 and Figure 7). As the width of the device is about16 in. (407 mm), the first and last measurements of each reconstruction were centered $12 \mathrm{in.}(304.8 \mathrm{~mm})$ from the edge, 
resulting in a total of 16 overlapping measurements within each extended reconstruction, covering the entire area of the specimen in the direction of the current set of scans.

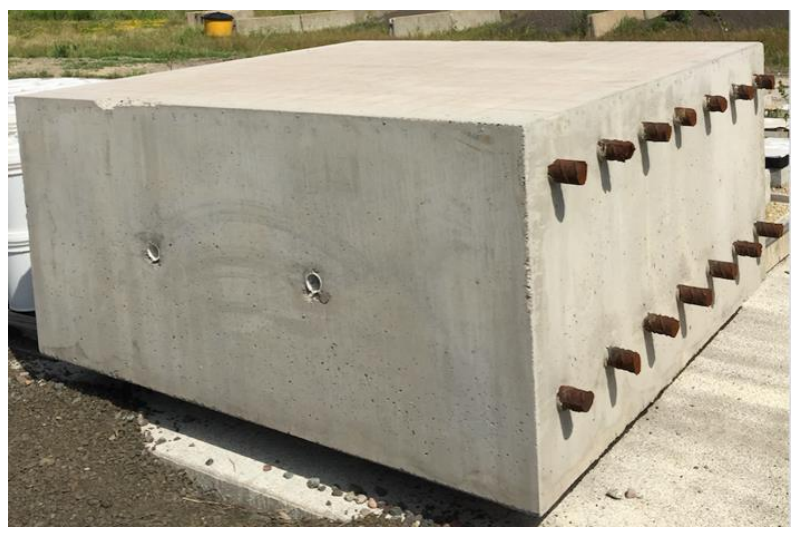

Figure 4. Concrete specimen.

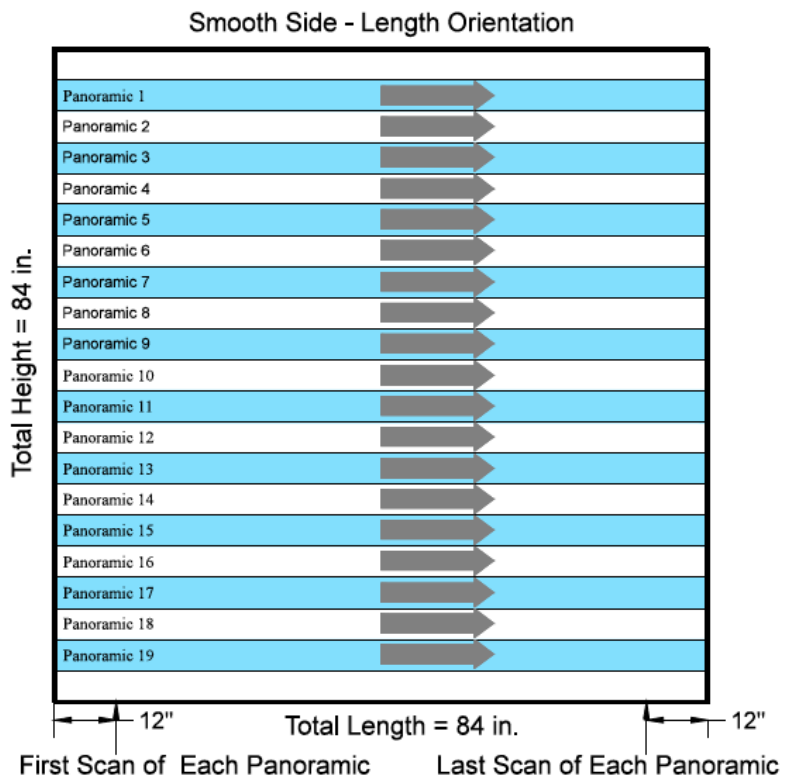

Figure 6. Scan direction along length orientation.

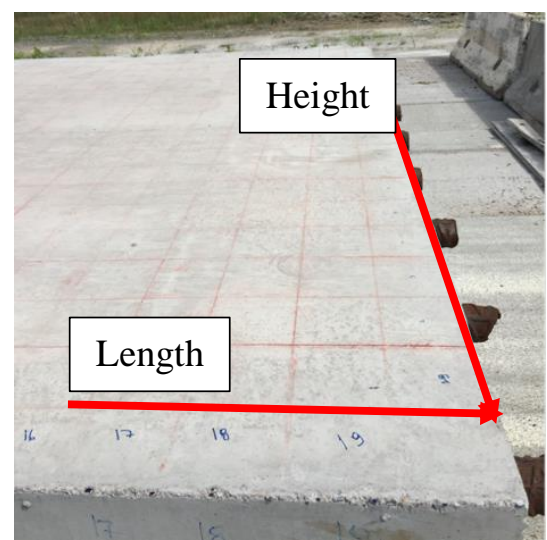

Figure 5. Scan directions on the smooth surface.

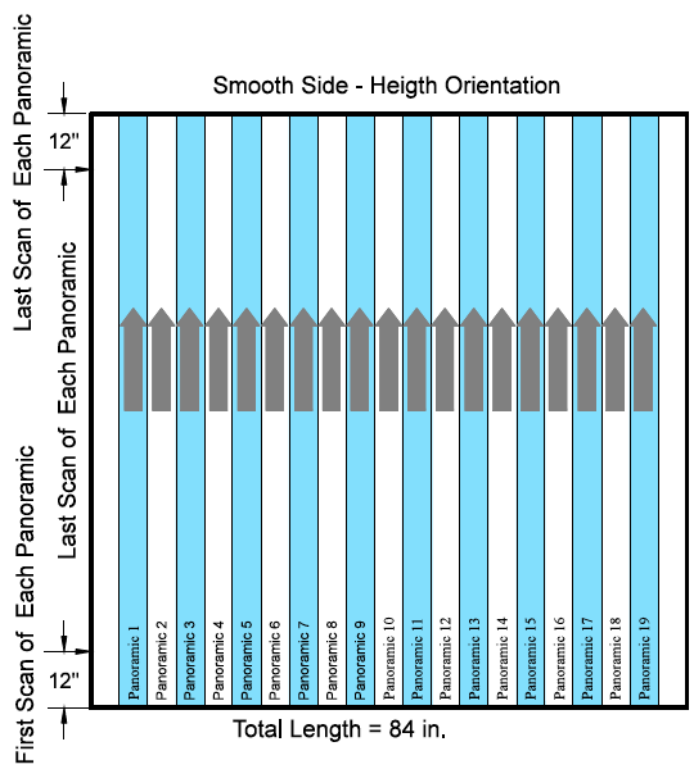

Figure 7. Scan direction along height orientation.

\section{MIRA DATA COLLECTION}

Two series of tests were performed in this study. The objective of the first series was to evaluate repeatability of the measurements and resulting reconstructed images. The ultrasound measurements were made along the length direction in rows 9 and 12. For each location, the following measurements were conducted: 
- five measurements with the target center frequency of the emitted signal of $50 \mathrm{kHz}$, two half-periods duration, without lifting the device from the surface;

- five measurements with the target center frequency of the emitted signal of $50 \mathrm{kHz}$, two half-periods duration, with lifting of the device from the surface;

- five measurements with the target center frequency of the emitted signal of $30 \mathrm{kHz}$, two half-periods duration, without lifting the device from the surface; and

- five measurements with the target center frequency of the emitted signal of $100 \mathrm{kHz}$, two half-periods duration, without lifting the device from the surface.

Table A1 in Appendix A summarizes the testing programs and provides the names of the corresponding row data files.

The objective of the second series of tests was to obtain measurements with various signal durations. The following tests were conducted for each row of the grid in the direction of length and height of the specimen on the smooth surface:

- $\quad$ one half-period duration of the emitted signal with the target center frequency of the emitted signal $50 \mathrm{kHz}$,

- two half-periods duration of the emitted signal with the target center frequency of the emitted signal $50 \mathrm{kHz}$,

- four half-periods duration of the emitted signal with the target center frequency of the emitted signal $50 \mathrm{kHz}$, and

- $\quad$ sixteen half-periods duration of the emitted signal with the target center frequency of the emitted signal $50 \mathrm{kHz}$.

For each impulse duration, only one measurement was conducted in this series. Table A2 in Appendix A summarizes the testing programs and provides the names of the corresponding row data files for this series of tests.

\subsection{PERLIMINARY DATA ANALYSIS}

The data collected from the large specimen were processed using software developed at the University of Minnesota. For each measurement, the following parameters were computed:

- $\quad$ surface shear wave velocity,

- Hilbert transform indicator (HTI) [2], and

- SAFT reflectivity matrix.

In addition, for each row of measurements, a panoramic reconstruction, SAFT-PAN [3] was created.

\subsection{SERIES 1: REPEATABILITY ANALYSIS}

Table 1 presents computed mean values and coefficients of variation of velocities from five measurements at each location of row 9. It is observed that for all locations, except locations 7 and 14, all four methods resulted in very consistent velocities with coefficients of variation less than $1 \%$. For 
location 14 , measurements at $50 \mathrm{kHz}$ lifting the device and measurements at $100 \mathrm{kHz}$ without lifting the device resulted in coefficients of variation of $4.7 \%$ and $3.8 \%$, respectively. Figure 8 displays a comparison of the velocities determined from testing with and without lifting the device. A good agreement is observed. Analysis of Table 1 also shows good agreement in velocities from all four testing methods for every location except location 14.Table 2 presents computed mean values and coefficients of variation of HTIs from five measurements at each location of row 9. HTI is defined as follows:

$$
H T I=\int_{0}^{500} \frac{H T(t)}{H T_{\max }} d t
$$

where $H T(t)$ is the Hilbert transform of a signal $f(t)$ defined as

$$
H T(t)=\sqrt{(f(t))^{2}+\left(\frac{1}{\pi} \int_{-\infty}^{\infty} \frac{f(\tau)}{t-\tau} d \tau\right)^{2}},
$$

$t$ is time, and HTmax is the maximum value of the function $H T(t)$ in the interval from 0 to $500 \mu \mathrm{s}$. HTI can be interpreted as an effective duration of the received signal. For the same frequency of the sent impulse a higher HTI suggests a worse condition of the surface. However, a change in the frequency or duration of the signal also affects HTI.

\begin{tabular}{|c|c|c|c|c|c|c|c|c|}
\hline \multirow[b]{2}{*}{ Location } & \multicolumn{4}{|c|}{ Mean Surface Velocity, km/s } & \multicolumn{4}{|c|}{ Coefficient of Variation } \\
\hline & $\begin{array}{l}50 \mathrm{kHz} \\
\text { without } \\
\text { lifting }\end{array}$ & $\begin{array}{c}50 \mathrm{kHz} \\
\text { with lifting }\end{array}$ & $\begin{array}{c}30 \mathrm{kHz} \\
\text { without } \\
\text { lifting }\end{array}$ & $\begin{array}{c}100 \mathrm{kHz} \\
\text { without } \\
\text { lifting }\end{array}$ & $\begin{array}{c}50 \mathrm{kHz} \\
\text { without } \\
\text { lifting } \\
\end{array}$ & $\begin{array}{c}50 \mathrm{kHz} \\
\text { with lifting }\end{array}$ & $\begin{array}{c}30 \mathrm{kHz} \\
\text { without } \\
\text { lifting }\end{array}$ & $\begin{array}{c}100 \mathrm{kHz} \\
\text { without } \\
\text { lifting }\end{array}$ \\
\hline 1 & 2.6448 & 2.6556 & 2.6566 & 2.6668 & 0.0027 & 0.0039 & 0.0023 & 0.0015 \\
\hline 2 & 2.6418 & 2.6578 & 2.653 & 2.6778 & 0.0032 & 0.0039 & 0.0027 & 0.0022 \\
\hline 3 & 2.6292 & 2.6058 & 2.5996 & 2.6002 & 0.0030 & 0.0207 & 0.0031 & 0.0034 \\
\hline 4 & 2.5086 & 2.5104 & 2.523 & 2.5074 & 0.0015 & 0.0057 & 0.0025 & 0.0034 \\
\hline 5 & 2.5258 & 2.5298 & 2.5248 & 2.5226 & 0.0034 & 0.0043 & 0.0028 & 0.0014 \\
\hline 6 & 2.5012 & 2.5132 & 2.508 & 2.506 & 0.0024 & 0.0038 & 0.0014 & 0.0014 \\
\hline 7 & 2.6288 & 2.5768 & 2.6668 & 2.6438 & 0.0021 & 0.0180 & 0.0024 & 0.0020 \\
\hline 8 & 2.6634 & 2.6654 & 2.6732 & 2.675 & 0.0039 & 0.0024 & 0.0016 & 0.0016 \\
\hline 9 & 2.6818 & 2.6774 & 2.6798 & 2.6818 & 0.0033 & 0.0025 & 0.0026 & 0.0027 \\
\hline 10 & 2.7038 & 2.732 & 2.7508 & 2.7126 & 0.0045 & 0.0072 & 0.0017 & 0.0009 \\
\hline 11 & 2.7174 & 2.7152 & 2.712 & 2.7104 & 0.0034 & 0.0036 & 0.0027 & 0.0037 \\
\hline 12 & 2.7036 & 2.7082 & 2.7252 & 2.6814 & 0.0051 & 0.0058 & 0.0013 & 0.0038 \\
\hline 13 & 2.7364 & 2.7288 & 2.7354 & 2.7216 & 0.0025 & 0.0066 & 0.0011 & 0.0011 \\
\hline 14 & 2.5884 & 2.6648 & 2.7746 & 2.6752 & 0.0017 & 0.0469 & 0.0033 & 0.0379 \\
\hline 15 & 2.792 & 2.8022 & 2.8058 & 2.8118 & 0.0028 & 0.0042 & 0.0013 & 0.0011 \\
\hline 16 & 2.802 & 2.8044 & 2.7942 & 2.8012 & 0.0017 & 0.0022 & 0.0018 & 0.0006 \\
\hline
\end{tabular}

Table 1. Statistical summary of computed surface velocities for each location and test method 


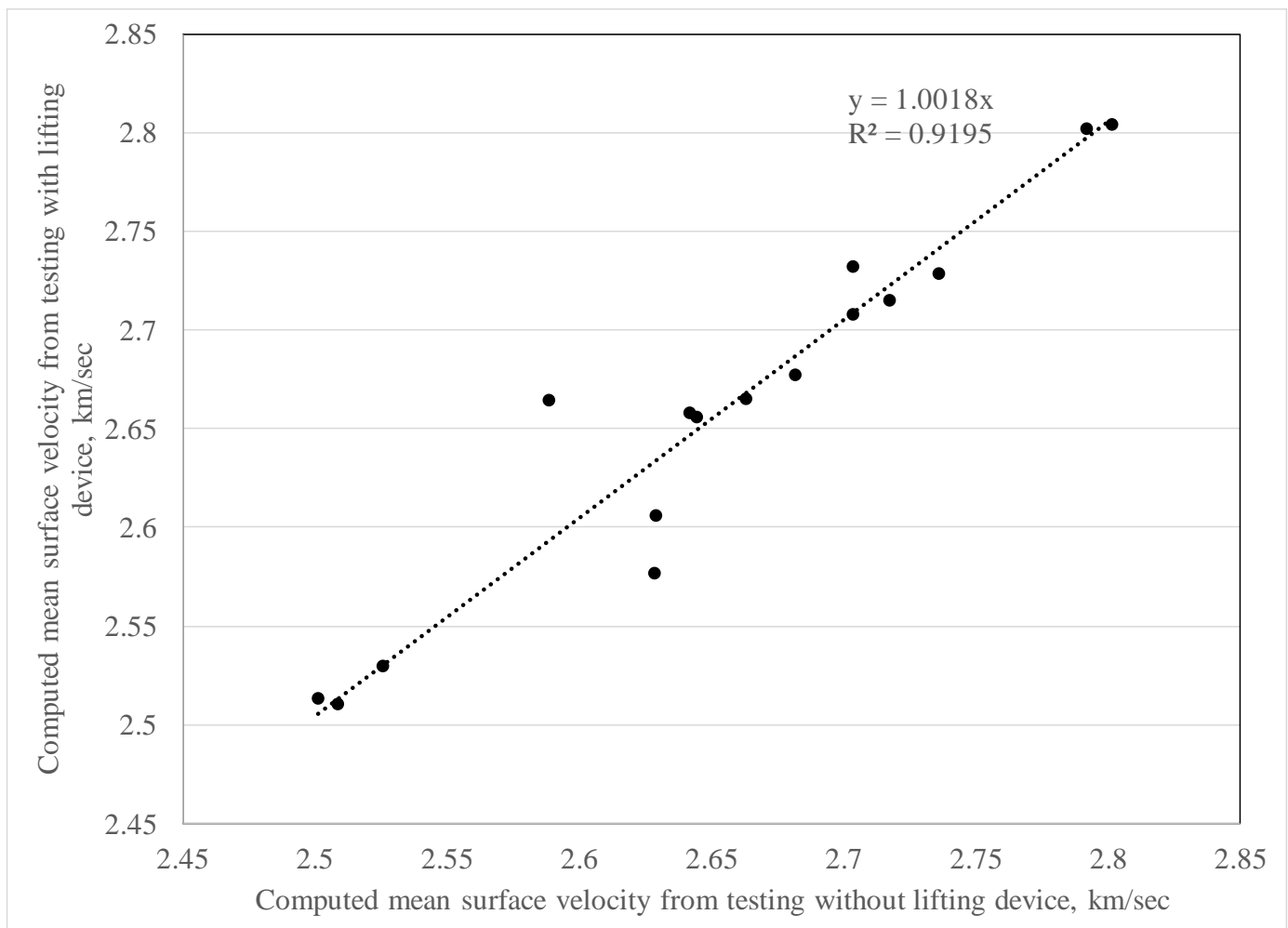

Figure 8. Mean surface velocities from testing with and without lifting the device.

Table 2. Statistical summary of the computed Hilbert transform indicators (HTIs) for each location and test method

\begin{tabular}{|c|c|c|c|c|c|c|c|c|}
\hline \multirow[b]{2}{*}{ Location } & \multicolumn{4}{|c|}{ Mean HTI } & \multicolumn{4}{|c|}{ Coefficient of Variation } \\
\hline & $\begin{array}{c}50 \mathrm{kHz} \\
\text { without } \\
\text { lifting }\end{array}$ & $\begin{array}{c}50 \mathrm{kHz} \\
\text { with } \\
\text { lifting }\end{array}$ & $\begin{array}{c}30 \mathrm{kHz} \\
\text { without } \\
\text { lifting }\end{array}$ & $\begin{array}{c}100 \mathrm{kHz} \\
\text { without } \\
\text { lifting }\end{array}$ & $\begin{array}{c}50 \mathrm{kHz} \\
\text { without } \\
\text { lifting }\end{array}$ & $\begin{array}{l}50 \mathrm{kHz} \\
\text { with } \\
\text { lifting }\end{array}$ & $\begin{array}{l}30 \mathrm{kHz} \\
\text { without } \\
\text { lifting }\end{array}$ & $\begin{array}{c}100 \mathrm{kHz} \\
\text { without } \\
\text { lifting }\end{array}$ \\
\hline 1 & 68.9768 & 68.8114 & 87.532 & 58.3498 & 0.0058 & 0.0148 & 0.0027 & 0.0035 \\
\hline 2 & 67.8942 & 67.656 & 82.8606 & 57.0168 & 0.0068 & 0.0291 & 0.0022 & 0.0121 \\
\hline 3 & 75.0688 & 77.1442 & 93.5608 & 66.2332 & 0.0065 & 0.0238 & 0.0031 & 0.0075 \\
\hline 4 & 78.479 & 79.6442 & 86.9058 & 80.0788 & 0.0067 & 0.0150 & 0.0031 & 0.0062 \\
\hline 5 & 78.5024 & 79.2284 & 88.9514 & 78.8708 & 0.0036 & 0.0175 & 0.0069 & 0.0022 \\
\hline 6 & 87.9426 & 89.2058 & 101.0784 & 84.02 & 0.0042 & 0.0179 & 0.0051 & 0.0062 \\
\hline 7 & 83.772 & 84.1246 & 102.479 & 75.4852 & 0.0121 & 0.0445 & 0.0067 & 0.0066 \\
\hline 8 & 70.8396 & 70.4994 & 94.874 & 59.0666 & 0.0051 & 0.0156 & 0.0035 & 0.0018 \\
\hline 9 & 62.187 & 63.6296 & 84.691 & 53.0034 & 0.0033 & 0.0139 & 0.0034 & 0.0134 \\
\hline 10 & 60.75 & 59.8848 & 79.0158 & 54.5186 & 0.0052 & 0.0320 & 0.0015 & 0.0142 \\
\hline 11 & 67.774 & 65.4844 & 87.8762 & 60.7272 & 0.0090 & 0.0567 & 0.0027 & 0.0067 \\
\hline 12 & 68.3082 & 68.0002 & 82.3052 & 61.4432 & 0.0061 & 0.0186 & 0.0016 & 0.0138 \\
\hline 13 & 63.7914 & 62.722 & 80.2468 & 55.69 & 0.0086 & 0.0161 & 0.0115 & 0.0077 \\
\hline 14 & 73.9228 & 71.2958 & 88.15 & 55.3818 & 0.0034 & 0.0478 & 0.0113 & 0.0060 \\
\hline 15 & 62.8264 & 63.0048 & 85.1546 & 50.0858 & 0.0046 & 0.0147 & 0.0031 & 0.0058 \\
\hline 16 & 61.7032 & 62.694 & 83.2412 & 48.5928 & 0.0037 & 0.0082 & 0.0020 & 0.0172 \\
\hline
\end{tabular}


One can see from Table 2 that testing without lifting the device resulted in very consistent HTIs with the coefficients of variation less than $1 \%$ for most locations. Testing with lifting the device resulted in a slightly higher variability with the coefficients of variation from $1 \%$ to $5 \%$. Reasonably good correspondence in mean values when testing with a frequency of $50 \mathrm{kHz}$, with or without lifting the device, is observed in the results in Table 2 and Figure 9. As expected, testing with different frequencies resulted in different HTIs.

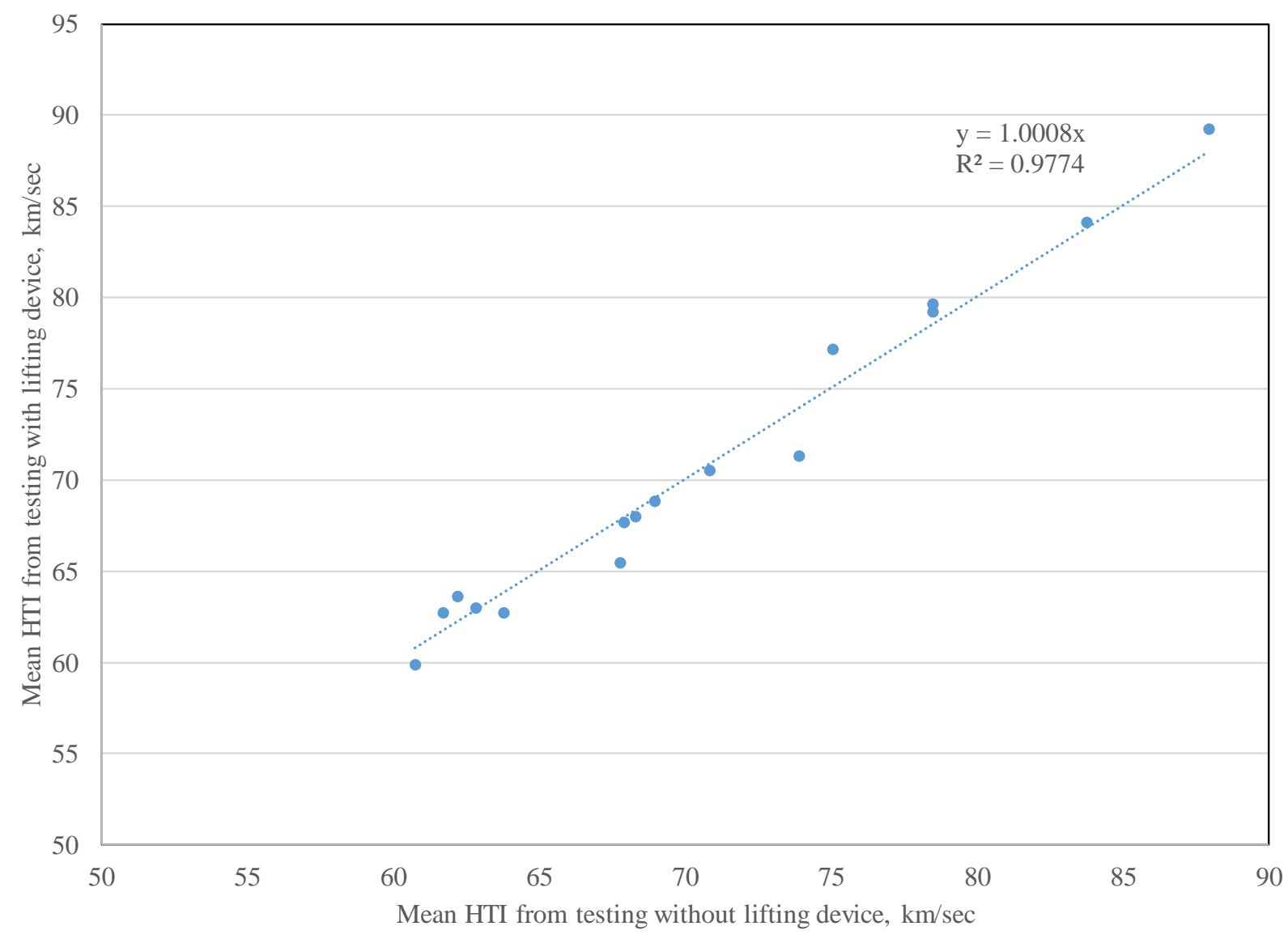

Figure 9. Mean Hilbert transform indicators (HTI) from testing with and without lifting the device.

For PCC reconstruction in 2-D (cross-sectional) with complex conditions, Kirchhoff-based migration has traditionally been used. Although the traditional SAFT method is simple and heuristically formulated, this technology has been successfully used for evaluation of various concrete infrastructures [4]. In this study, for each measurement an instantaneous amplitude SAFT B-scan (SAFT-IA) was computed. Because the magnitude of the recorded signals and the intensity of the SAFT-IA reconstruction may depend on the pressure applied to the device during measurement, some variability in intensity of the SAFT-IA reconstructions from different measurements is expected.

Figure 10 shows SAFT-IA reconstructions for location 4, row 9. The reconstructions from all the measurements, when the device was not lifted, look very similar. Reconstructions for location 7, row 9 (Figure 11) look very similar for the measurements without lifting the device, but less similar when the device was lifted. Reconstructions for location 14, row 9 (Figure 12) demonstrate a greater degree of dissimilarity. 

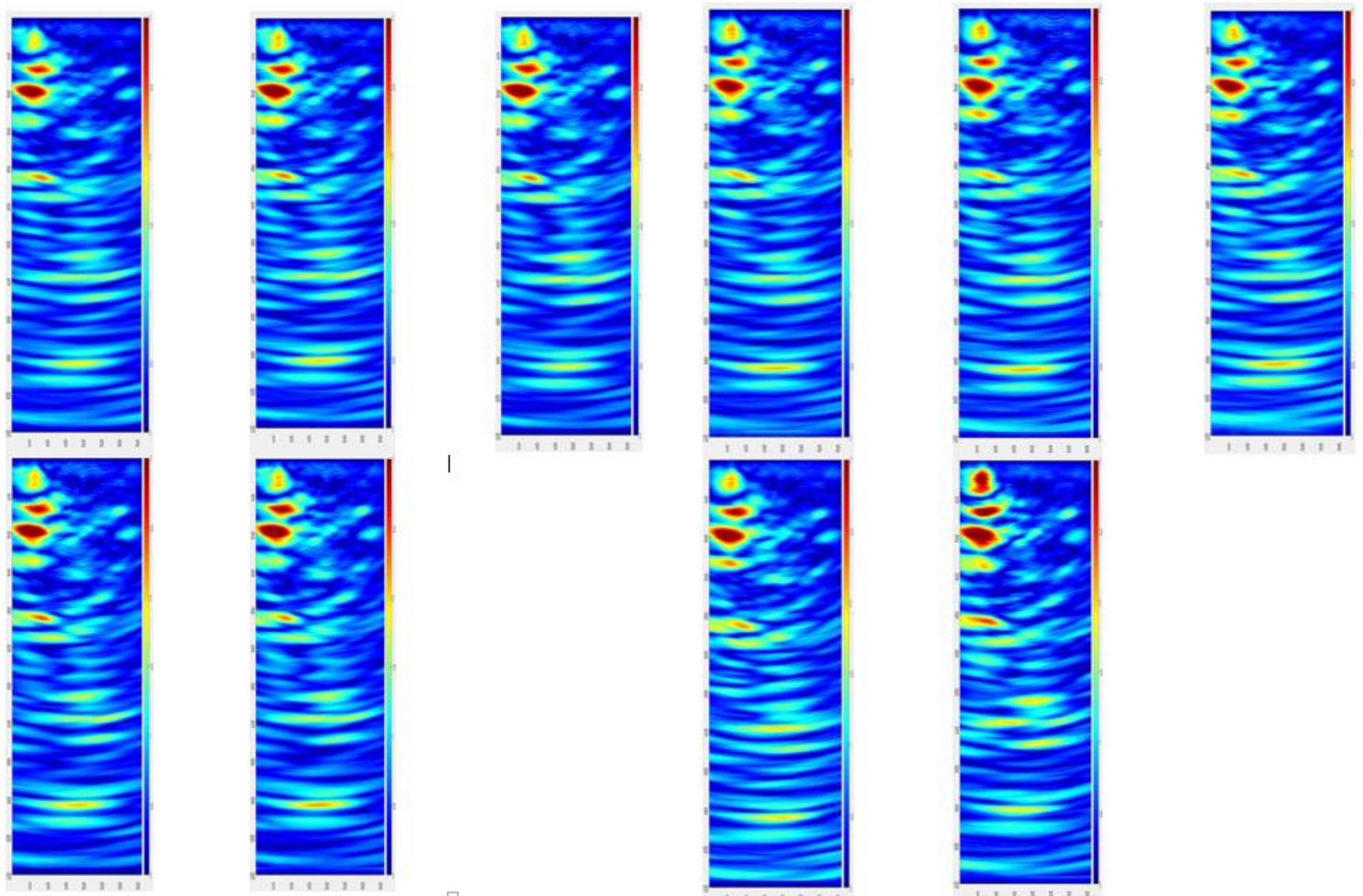

(a)

(b)

Figure 10. SAFT-IA reconstructions for location 4, row 9: (a) without lifting the device and (b) with lifting the device. 

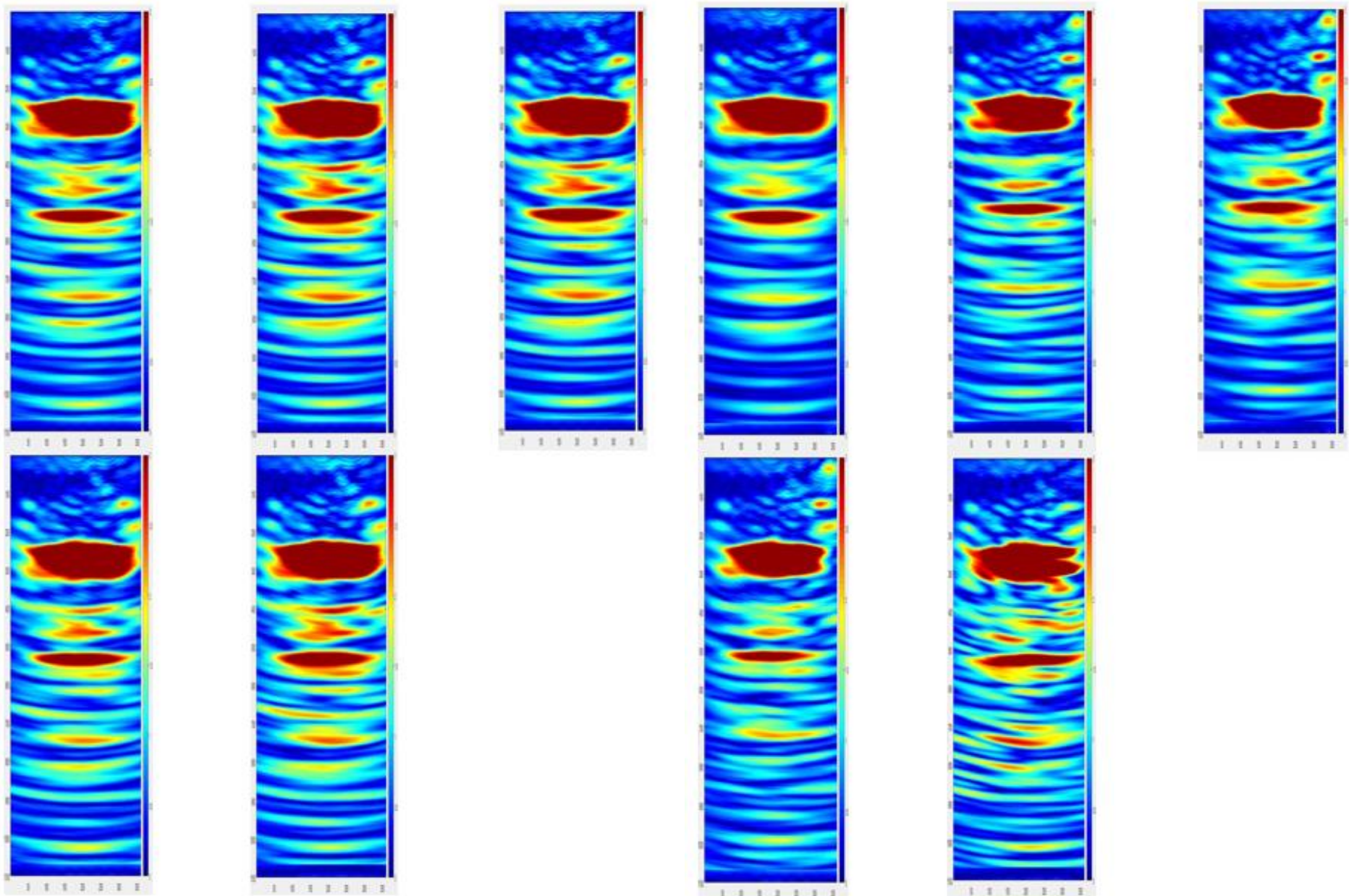

(b)

Figure 11. SAFT-IA reconstructions for location 7, row 9: (a) without lifting the device and (b) with lifting the device. 

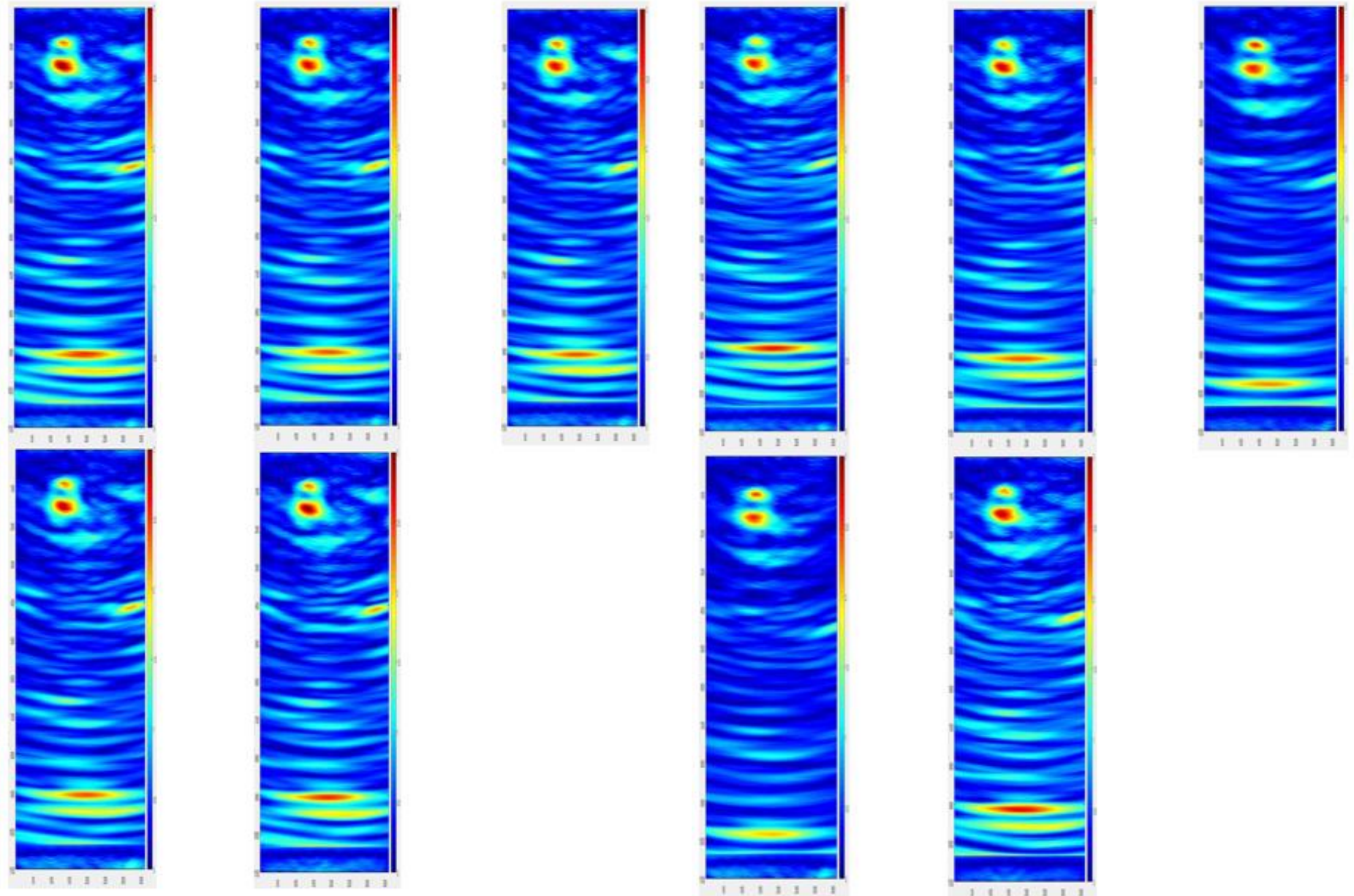

(a)

(b)

Figure 12. SAFT-IA reconstructions for location 14, row 9 (a) without lifting the device and (b) with lifting the device 
In this study, the Pearson's correlation coefficient was adapted for quantifying similarity of the reconstructed intensity matrices. For each location and test type, the reconstructed intensity matrix corresponding to the first SAFT-IA was considered as the reference scan, and the remaining four SAFTIA Pearson's correlation coefficients for the remaining scans were computed as follows:

$$
C^{I A, m}=\frac{\operatorname{Cov}\left[[\hat{\boldsymbol{o}}]^{I A, r e f},[\widehat{\boldsymbol{o}}]^{I A, m}\right]}{\sqrt{\operatorname{Var}\left[[\hat{\boldsymbol{o}}]^{I A, r e f}\right] \operatorname{Var}\left[[\widehat{\boldsymbol{o}}]^{I A, m}\right]}}=\frac{\sum_{i=1}^{W} \sum_{k=1}^{D}\left(\widehat{\boldsymbol{o}}_{i, k}^{I A, r e f}-\widehat{\boldsymbol{o}}_{m e a n}^{I A, r e f}\right)\left(\widehat{\boldsymbol{o}}_{i, k}^{I A, m}-\widehat{\boldsymbol{o}}_{\text {mean }}^{I A, m}\right)}{\sum_{i=1}^{W} \sum_{k=1}^{D}\left(\widehat{\boldsymbol{o}}_{i, k}^{I A, r e f}-\widehat{\boldsymbol{o}}_{\text {mean }}^{I A, r e f}\right)^{2} \sum_{i=1}^{W} \sum_{k=1}^{D}\left(\widehat{\boldsymbol{o}}_{i, k}^{I A}-\widehat{\boldsymbol{o}}_{\text {mean }}^{I A}\right)^{2}}
$$

where $[\widehat{\boldsymbol{O}}]^{I \text { Aref }}$ and $[\widehat{\boldsymbol{O}}]^{I A, m}$ are the matrices of reflection intensity for the reference SAFT-IA B-scan and $m$-th SAFT-IA B-scan. Also; $\widehat{\boldsymbol{o}}_{i, k}^{I A, r e f}$ and $\widehat{\boldsymbol{o}}_{i, k}^{I A}$ are the single intensity values of the reference signal and $m$-th reconstruction, respectively, with depth below the measurement location increasing with $k$ and the location along the horizontal direction of the scan increasing with $i$; $\widehat{\boldsymbol{o}}_{\text {mean }}^{I A, r e f}$ and $\widehat{\boldsymbol{o}}_{\text {mean }}^{I A}$ are the mean intensities of the reference scan and $m$-th scan. Finally, $W$ and $D$ are the numbers of width and depth intensity values in the depth and device aperture direction, respectively, and $C^{I A, m}$ is Pearson's correlation coefficient, which measures the strength of the linear dependence between $[\widehat{\boldsymbol{O}}]^{\text {IAref }}$ and $[\widehat{\boldsymbol{O}}]^{I A, m}$.

If there is a strong positive linear correlation between the scans, then the Pearson's correlation coefficient is close to one. Experience shows that similar scans from field-measured data should exhibit a Pearson's correlation coefficient greater than 0.9 [5]. If the Pearson's correlation coefficient is close to 0 , then there is no linear correlation between the scans.

The resulting Pearson's correlation coefficients for each test type are given in Table 3 through Table 6 for each location in row 9 and two reconstruction depths: $500 \mathrm{~mm}$ and 1,100 $\mathrm{mm}$. A high level of correlation is observed for $50 \mathrm{kHz}$ and $30 \mathrm{kHz}$ testing without lifting the device, with all the scans resulting in Pearson's correlation coefficients greater than 0.9 , suggesting high similarity with the first scan for the corresponding locations. Although the correlation is lower for the $50 \mathrm{kHz}$ and $100 \mathrm{kHz}$ testing without lifting the device, the correlation is reasonably high for all locations except location 14.

Table 3 through Table 6 also show that an increase in the reconstruction depth leads to reduction in the correlation coefficients for all locations and test types. Nevertheless, testing with the frequency equal to $30 \mathrm{kHz}$ resulted in high correlation for all locations except 14 . Testing with the frequency equal to $50 \mathrm{kHz}$ resulted in high correlation for 56 out of 64 SAFT-IAs. At the same time, testing with $50 \mathrm{kHz}$ with lifting of the device and testing with $100 \mathrm{kHz}$ without lifting the device results in low correlations for a significant percentage of the measurements. This is explained by an increased noise-to-signal ratio for the reflections with higher depth and increased frequency. 
Table 3. Pearson correlation coefficients for $50 \mathrm{kHz}$ without lifting

\begin{tabular}{lllllllll}
\hline \multirow{2}{*}{ Location } & \multicolumn{3}{c}{$\mathbf{5 0 0}$ mm reconstruction depth } & \multicolumn{3}{c}{$\mathbf{1 , 1 0 0}$ mm reconstruction depth } \\
\cline { 2 - 9 } & Scan 2 & Scan 3 & Scan 4 & Scan $\mathbf{5}$ & Scan 2 & Scan 3 & Scan 4 & Scan 5 \\
\hline 1 & 0.9805 & 0.9663 & 0.9784 & 0.9667 & 0.9717 & 0.9593 & 0.9745 & 0.9735 \\
2 & 0.9442 & 0.9782 & 0.9664 & 0.9515 & 0.9202 & 0.9646 & 0.9607 & 0.9395 \\
3 & 0.9662 & 0.9553 & 0.9695 & 0.9800 & 0.8950 & 0.8869 & 0.9344 & 0.9525 \\
4 & 0.9792 & 0.9817 & 0.9804 & 0.9814 & 0.9576 & 0.9471 & 0.9615 & 0.9552 \\
5 & 0.9844 & 0.9743 & 0.9790 & 0.9720 & 0.9421 & 0.9234 & 0.9471 & 0.8998 \\
6 & 0.9953 & 0.9956 & 0.9962 & 0.9930 & 0.9898 & 0.9837 & 0.9919 & 0.9785 \\
7 & 0.9946 & 0.9966 & 0.9962 & 0.9960 & 0.9812 & 0.9922 & 0.9924 & 0.9879 \\
8 & 0.9961 & 0.9967 & 0.9959 & 0.9954 & 0.9874 & 0.9929 & 0.9889 & 0.9825 \\
9 & 0.9760 & 0.9705 & 0.9895 & 0.9853 & 0.9301 & 0.9280 & 0.9691 & 0.9543 \\
10 & 0.9402 & 0.9784 & 0.9386 & 0.9186 & 0.8616 & 0.9551 & 0.8611 & 0.8204 \\
11 & 0.9808 & 0.9898 & 0.9913 & 0.9879 & 0.9474 & 0.9819 & 0.9854 & 0.9759 \\
12 & 0.9482 & 0.9822 & 0.9851 & 0.9853 & 0.7973 & 0.9672 & 0.9687 & 0.9690 \\
13 & 0.9608 & 0.9392 & 0.9375 & 0.9292 & 0.9641 & 0.8975 & 0.9321 & 0.9084 \\
14 & 0.9732 & 0.9544 & 0.9782 & 0.9878 & 0.9637 & 0.9204 & 0.9737 & 0.9769 \\
15 & 0.9860 & 0.9895 & 0.9845 & 0.9790 & 0.9689 & 0.9845 & 0.9787 & 0.9529 \\
16 & 0.9871 & 0.9844 & 0.9854 & 0.9758 & 0.9757 & 0.9615 & 0.9652 & 0.9449 \\
\hline
\end{tabular}

Table 4. Pearson correlation coefficients for $50 \mathrm{kHz}$ with lifting

\begin{tabular}{llllllllll}
\hline \multirow{2}{*}{ Location } & \multicolumn{3}{c}{$\mathbf{5 0 0}$ mm reconstruction depth } & \multicolumn{3}{c}{ 1.100 mm reconstruction depth } \\
\cline { 2 - 9 } & Scan 2 & Scan 3 & Scan 4 & Scan 5 & Scan 2 & Scan 3 & Scan 4 & Scan 5 \\
\hline 1 & 0.9175 & 0.9144 & 0.9068 & 0.8878 & 0.9448 & 0.8797 & 0.8482 & 0.8388 \\
2 & 0.9274 & 0.8728 & 0.8880 & 0.8309 & 0.8826 & 0.8546 & 0.9262 & 0.8829 \\
3 & 0.9239 & 0.6169 & 0.9093 & 0.9304 & 0.9287 & 0.3229 & 0.9102 & 0.9183 \\
4 & 0.9797 & 0.9560 & 0.9633 & 0.9337 & 0.9308 & 0.8942 & 0.9261 & 0.8126 \\
5 & 0.9458 & 0.9352 & 0.9449 & 0.9547 & 0.9268 & 0.9062 & 0.9109 & 0.8869 \\
6 & 0.9762 & 0.9769 & 0.9676 & 0.9762 & 0.9341 & 0.9640 & 0.9351 & 0.9685 \\
7 & 0.8727 & 0.8607 & 0.8680 & 0.9387 & 0.7418 & 0.7425 & 0.7435 & 0.8888 \\
8 & 0.9816 & 0.9913 & 0.9847 & 0.9831 & 0.9768 & 0.9872 & 0.9791 & 0.9798 \\
9 & 0.9679 & 0.8640 & 0.8901 & 0.9503 & 0.9443 & 0.8293 & 0.8392 & 0.9119 \\
10 & 0.8269 & 0.8103 & 0.9128 & 0.9346 & 0.7342 & 0.7572 & 0.8896 & 0.9277 \\
11 & 0.9770 & 0.9485 & 0.9067 & 0.8688 & 0.9689 & 0.9321 & 0.9157 & 0.8803 \\
12 & 0.9116 & 0.8823 & 0.8879 & 0.9177 & 0.7058 & 0.7842 & 0.6248 & 0.8116 \\
13 & 0.8722 & 0.9661 & 0.9652 & 0.9497 & 0.6321 & 0.9560 & 0.9265 & 0.9491 \\
14 & 0.7498 & 0.4768 & 0.4812 & 0.6873 & 0.3631 & 0.2560 & 0.2135 & 0.3659 \\
15 & 0.9372 & 0.9452 & 0.9564 & 0.9576 & 0.6293 & 0.9322 & 0.9431 & 0.9318 \\
16 & 0.9240 & 0.9556 & 0.9553 & 0.9408 & 0.8918 & 0.9558 & 0.9562 & 0.9479 \\
\hline
\end{tabular}


Table 5. Pearson correlation coefficients for $30 \mathrm{kHz}$ without lifting

\begin{tabular}{lllllllll}
\hline \multirow{2}{*}{ Location } & \multicolumn{3}{c}{$\mathbf{5 0 0}$ mm reconstruction depth } & \multicolumn{3}{c}{ 1,100 mm reconstruction depth } \\
\cline { 2 - 9 } & Scan 2 & Scan 3 & Scan 4 & Scan 5 & Scan 2 & Scan 3 & Scan 4 & Scan 5 \\
\hline 1 & 0.9905 & 0.9892 & 0.9740 & 0.9891 & 0.9761 & 0.9729 & 0.9561 & 0.9769 \\
2 & 0.9885 & 0.9910 & 0.9893 & 0.9882 & 0.9853 & 0.9638 & 0.9796 & 0.9696 \\
3 & 0.9868 & 0.9822 & 0.9670 & 0.9896 & 0.9656 & 0.9572 & 0.9316 & 0.9779 \\
4 & 0.9934 & 0.9899 & 0.9876 & 0.9875 & 0.9832 & 0.9735 & 0.9609 & 0.9574 \\
5 & 0.9935 & 0.9908 & 0.9872 & 0.9838 & 0.9788 & 0.9749 & 0.9672 & 0.9572 \\
6 & 0.9920 & 0.9982 & 0.9980 & 0.9990 & 0.9840 & 0.9960 & 0.9906 & 0.9975 \\
7 & 0.9990 & 0.9984 & 0.9990 & 0.9993 & 0.9977 & 0.9945 & 0.9982 & 0.9983 \\
8 & 0.9981 & 0.9973 & 0.9977 & 0.9970 & 0.9953 & 0.9955 & 0.9925 & 0.9950 \\
9 & 0.9961 & 0.9894 & 0.9921 & 0.9907 & 0.9881 & 0.9611 & 0.9810 & 0.9794 \\
10 & 0.9613 & 0.9651 & 0.9554 & 0.9557 & 0.9559 & 0.9508 & 0.9344 & 0.9404 \\
11 & 0.9917 & 0.9908 & 0.9878 & 0.9894 & 0.9801 & 0.9801 & 0.9664 & 0.9704 \\
12 & 0.9966 & 0.9969 & 0.9956 & 0.9958 & 0.9872 & 0.9891 & 0.9831 & 0.9842 \\
13 & 0.9428 & 0.9489 & 0.9435 & 0.9465 & 0.9442 & 0.9398 & 0.9333 & 0.9488 \\
14 & 0.9741 & 0.9663 & 0.9626 & 0.9678 & 0.8404 & 0.7808 & 0.7691 & 0.8473 \\
15 & 0.9923 & 0.9901 & 0.9858 & 0.9877 & 0.9839 & 0.9831 & 0.9748 & 0.9737 \\
16 & 0.9954 & 0.9956 & 0.9929 & 0.9882 & 0.9941 & 0.9947 & 0.9892 & 0.9859 \\
\hline
\end{tabular}

Table 6. Pearson correlation coefficients for $100 \mathrm{kHz}$ without lifting

\begin{tabular}{llllllllll}
\hline \multirow{2}{*}{ Location } & \multicolumn{3}{c}{$\mathbf{5 0 0}$ mm reconstruction depth } & \multicolumn{3}{c}{ 1,100 mm reconstruction depth } \\
\cline { 2 - 9 } & Scan 2 & Scan 3 & Scan 4 & Scan $\mathbf{5}$ & Scan 2 & Scan 3 & Scan 4 & Scan 5 \\
\hline 1 & 0.9130 & 0.9064 & 0.9140 & 0.9312 & 0.9051 & 0.8999 & 0.9103 & 0.9203 \\
2 & 0.8564 & 0.9348 & 0.9223 & 0.9139 & 0.8165 & 0.8943 & 0.8920 & 0.8356 \\
3 & 0.8299 & 0.8385 & 0.8669 & 0.8657 & 0.7470 & 0.8263 & 0.7893 & 0.7833 \\
4 & 0.9646 & 0.9309 & 0.9515 & 0.9633 & 0.8780 & 0.8132 & 0.8599 & 0.8760 \\
5 & 0.9796 & 0.9808 & 0.9740 & 0.9698 & 0.9462 & 0.9376 & 0.9305 & 0.9363 \\
6 & 0.9649 & 0.9502 & 0.9526 & 0.9552 & 0.9302 & 0.8824 & 0.9015 & 0.9110 \\
7 & 0.9941 & 0.9893 & 0.9908 & 0.9903 & 0.9809 & 0.9411 & 0.9730 & 0.9678 \\
8 & 0.9887 & 0.9871 & 0.9897 & 0.9809 & 0.9712 & 0.9633 & 0.9726 & 0.9544 \\
9 & 0.9624 & 0.9230 & 0.9288 & 0.9460 & 0.8931 & 0.7971 & 0.8009 & 0.8358 \\
10 & 0.9062 & 0.9184 & 0.9235 & 0.9278 & 0.7603 & 0.7948 & 0.7739 & 0.8092 \\
11 & 0.8439 & 0.8627 & 0.8885 & 0.9057 & 0.7405 & 0.6901 & 0.7594 & 0.8196 \\
12 & 0.9450 & 0.8881 & 0.9457 & 0.9513 & 0.8980 & 0.7529 & 0.8674 & 0.8662 \\
13 & 0.9628 & 0.9650 & 0.9401 & 0.9411 & 0.9180 & 0.9187 & 0.9036 & 0.9091 \\
14 & 0.4230 & 0.4449 & 0.4517 & 0.8877 & 0.3662 & 0.3412 & 0.3361 & 0.7608 \\
15 & 0.9658 & 0.9557 & 0.9568 & 0.9628 & 0.9342 & 0.9214 & 0.9107 & 0.9115 \\
16 & 0.9454 & 0.9048 & 0.9044 & 0.9293 & 0.9269 & 0.9059 & 0.8964 & 0.9269 \\
\hline
\end{tabular}

The results of this analysis suggest that a linear array ultrasound system can produce reliably repeatable reconstructions using $50 \mathrm{kHz}$ signals for relatively shallow depths (less than $0.5 \mathrm{~m}$ ). However, for 
reconstructions at greater depths the use of lower frequency and/or signal filtering to reduce the effect of signal noise will be required.

\subsection{SERIES 3: SIGNAL DURATION IMFLUENCE}

For each row of measurements, a panoramic reconstruction, SAFT-PAN [3], was created. Appendix B presents the obtained reconstructions.

Figure 13 is a comparison of SAFT-PANs created in this study with SAFT-PANs reported by [1] for rows 7,8 , and 9 in the direction of specimen length with two half-periods durations. Figure 13 demonstrates that the scans obtained in this study are similar to those reported in previous reports [1]. Both sets of reconstructions clearly confirm the presence of a defect in rows 8 and 9. This defect, denoted as D13, was created by Styrofoam to simulate the presence of a void or poor consolidation of concrete [1]. Figure 14 displays design drawings for defect D13.
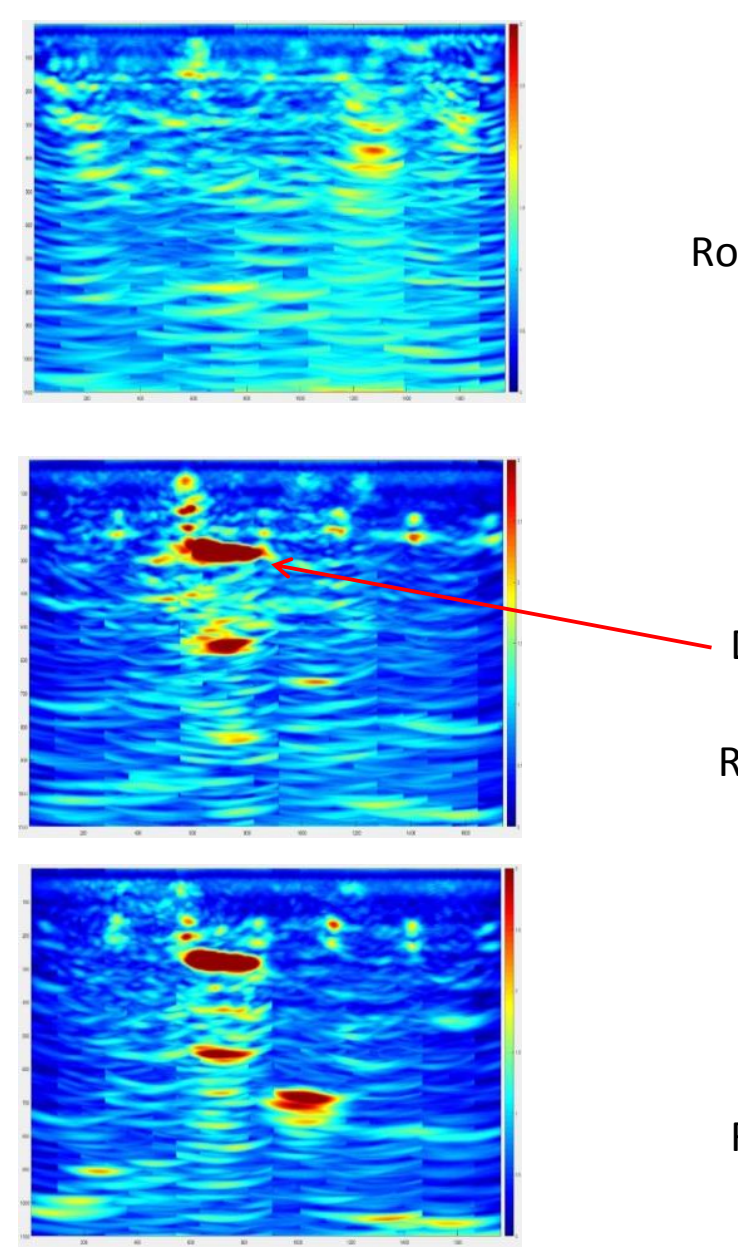

(a)

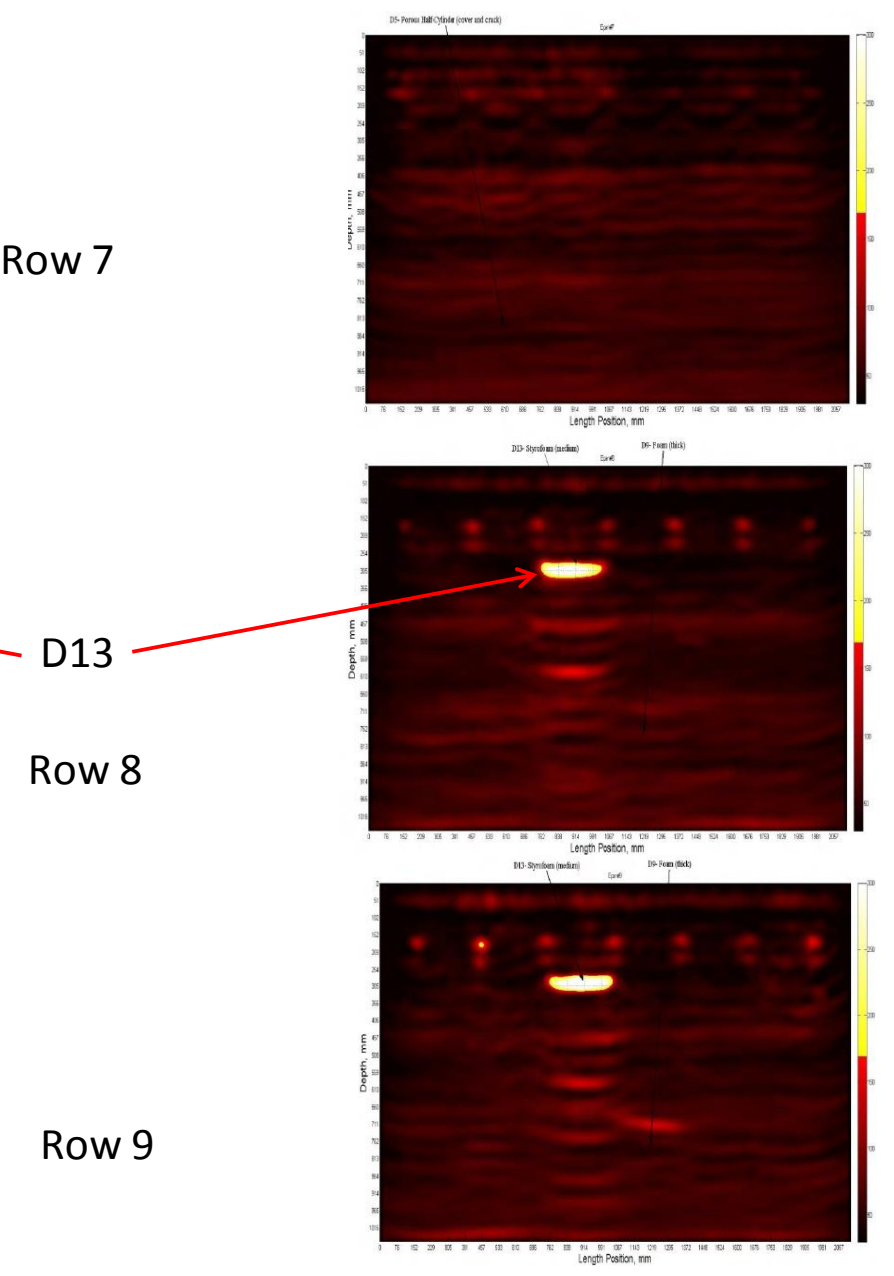

(b)

Figure 13. Comparison of panoramic reconstructions obtained in this study (a) with those reported in a previous study [1] (b). 


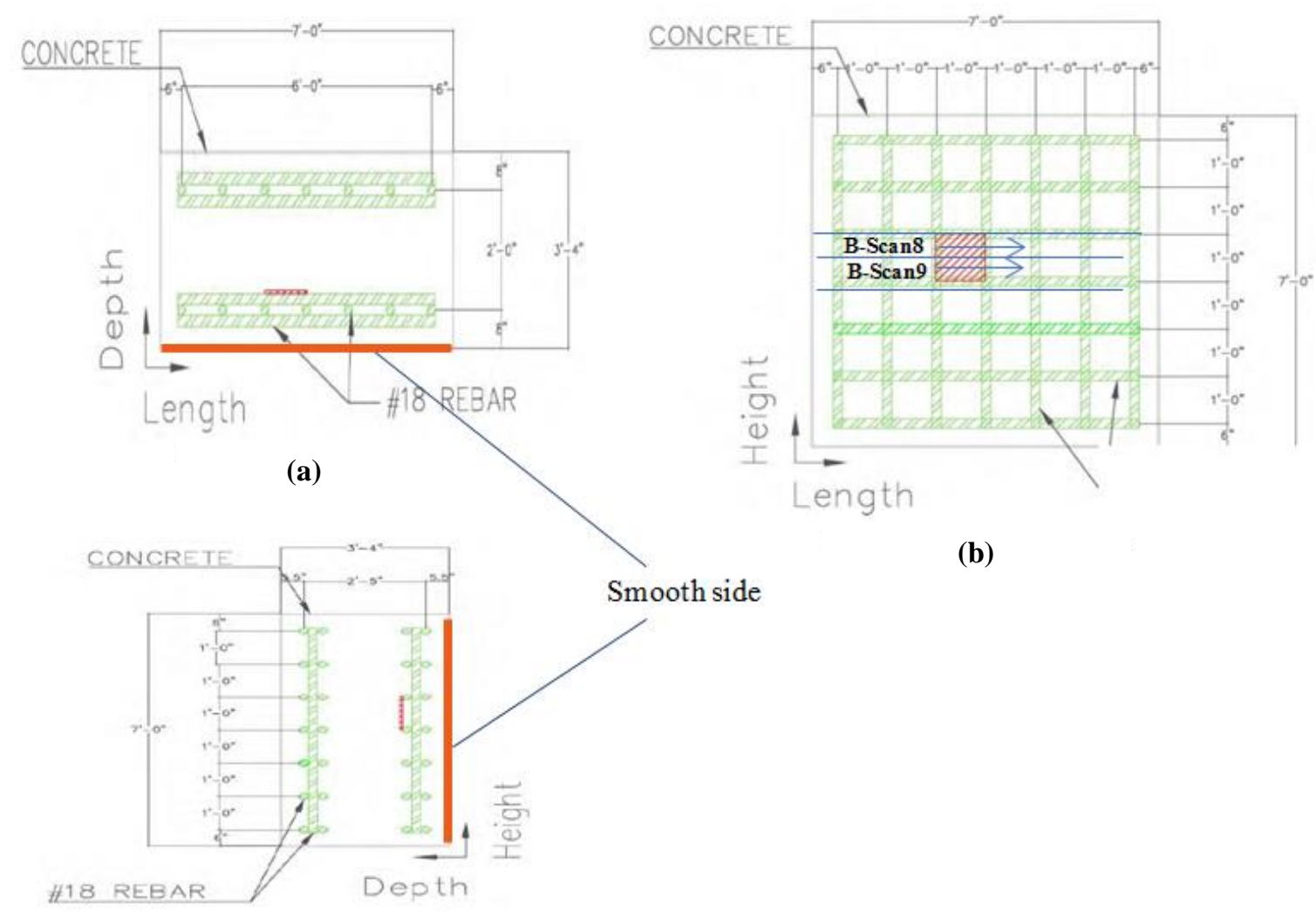

(c)

Figure 14. Location of the D13 defect and direction of the panoramic B-scan, rows 8 and 9, length direction: (a) D13 length by depth NL, (b) D13 length by height NL, and (c) D13 height by depth NL [1].

Panoramic reconstructions for row 8 obtained from testing with different impulse durations are displayed in Figure 15. The reconstructions from the one-half-period and two-half-period durations are very similar, clearly showing the presence of the D13 defect and of the reinforcement. Interpretation of the SAFT-PAN reconstruction from the four-half-period duration is less intuitive. The SAFT-PAN reconstruction from the 16-half-period signal duration is quite different from all other reconstructions. This suggests that Kirchhoff-based migration leads to more easily interpreted reconstructions when a shorter duration impulse signal is used. However, longer duration impulses may provide useful information for modelbased reconstructions, such as reverse time migration-based. A tool for obtaining such reconstructions is currently under development by the University of Minnesota team. 


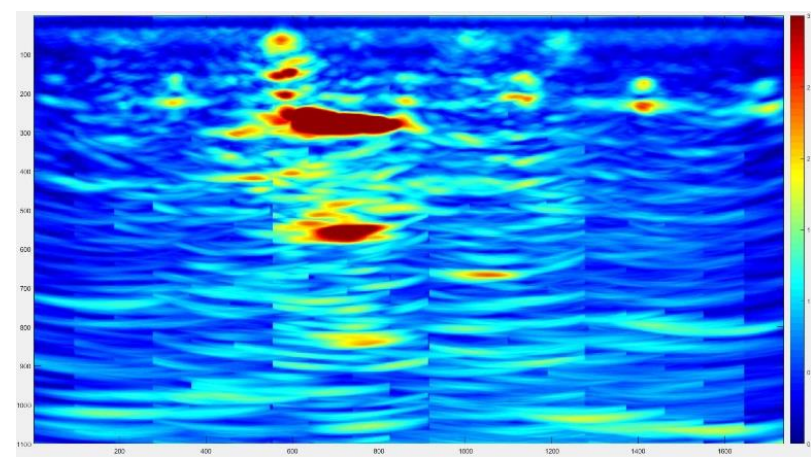

(a)

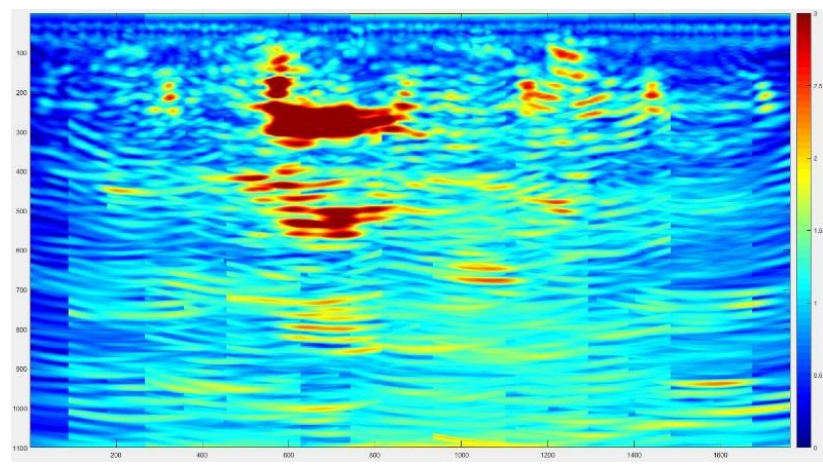

(c)

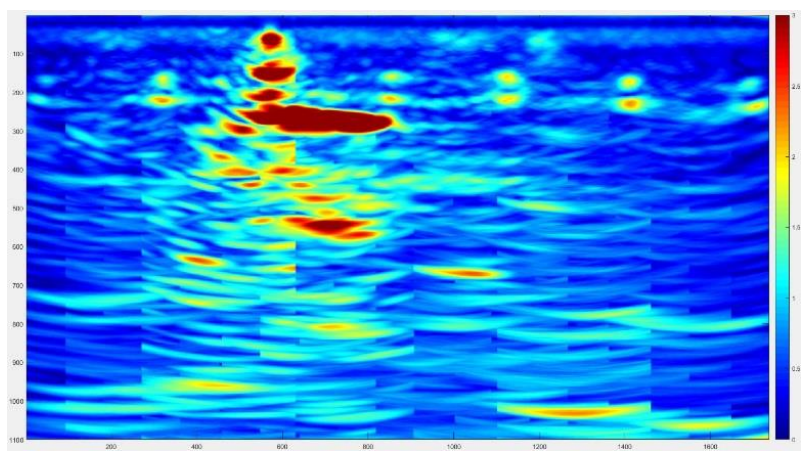

(b)

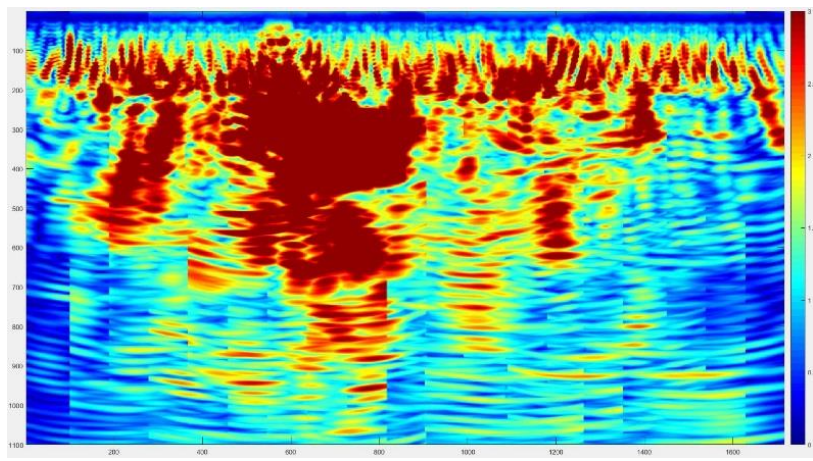

(d)

Figure 15. Panoramic reconstructions for row 8 with different impulse durations: (a) 1 half-period, (b) 2 halfperiods, (c) 4 half-periods, and (d) 16 half-periods. 


\section{CONCLUSIONS AND RECOMMENDATIONS}

In this study two series of ultrasound tests were performed on a thick concrete specimen with sufficient reinforcement density and size to represent an LWR containment wall with various defects. The objective of the first series was to evaluate repeatability of the measurements and resulting reconstructed images. Measurements were made with three center frequencies. Five measurements were made at each location with and without lifting the device. The objective of the second series of tests was to obtain measurements with various impulse signal durations. The entire grid on the smooth surface was tested with four different impulse signal durations.

A preliminary analysis of the collected data suggested that a linear array ultrasound system could produce reliably repeatable reconstructions using $50 \mathrm{kHz}$ signals for relatively shallow depths (less than $0.5 \mathrm{~m}$ ). However, for reconstructions at greater depths the use of lower frequency and/or signal filtering to reduce the effect of signal noise may be required. A comparison of the extended SAFT reconstructions obtained from measurements with various impulse signal durations suggested that Kirchhoff-based migration leads to more easily interpreted reconstructions when shorter duration impulse signals are used. Longer duration impulses can provide useful information for model-based reconstructions.

A more comprehensive analysis of the collected data is recommended. Developing a procedure for combining the reconstructions obtained with various impulse central frequencies and durations may lead to a more reliable characterization of subsurface conditions than individual reconstructions currently provide. It would also be good to document the acoustic speed of the specimen, and consequently the wavelength, to assess the impact of the cycles on depth; however, the signals from the thick specimen are not the best source of information for this type of analysis. Future work could consist of building concrete specimens of various thicknesses without defects and reinforcement and collecting data on these specimens.

\section{REFERENCES}

[1] Clayton, D. A., D. K. Hoegh, and D. L. Khazanovich. 2015. Thick Concrete Specimen Construction, Testing, and Preliminary Analysis (No. ORNL/TM-2015/72). Oak Ridge National Laboratory (ORNL).

[2] Freeseman, K., K. Hoegh, and L. Khazanovich. 2015. "Characterization of Concrete at Various Freeze-Thaw Damage Conditions Using SH-Waves." Proceedings of the 42nd Review of Progress in Quantitative Nondestructive Evaluation (QNDE), vol. July, pp. 26-31.

[3] Hoegh, K. and L. Khazanovich. 2015. "Extended Synthetic Aperture Focusing Technique for Ultrasonic Imaging of Concrete." NDT \& E International 74: 33:42.

[4] Schickert, M., M. Krause, and W. Müller. 2003. "Ultrasonic imaging of concrete elements using reconstruction by synthetic aperture focusing technique." Journal of Materials in Civil Engineering 15: $235-246$.

[5] Hoegh, K. and L. Khazanovich. 2012. "Correlation Analysis of 2D Tomographic Images for Flaw Detection in Pavements." Journal of Testing and Evaluation. American Society for Testing and Materials 40(2): 247-255. 

APPENDIX A. FILE NAMES AND TEST PROGRAM 



\section{APPENDIX A. FILE NAMES AND TEST PROGRAM}

Table A1. File names and test protocols for the first series of testing

\begin{tabular}{|l|l|l|l|l|l|l|}
\hline \multicolumn{1}{|c|}{ File name } & Row & $\begin{array}{c}\text { Half- } \\
\text { period }\end{array}$ & Orientation & $\begin{array}{c}\text { Frequency, } \\
\text { kHz }\end{array}$ & Lifting & $\begin{array}{c}\text { Number of scans } \\
\text { for each location }\end{array}$ \\
\hline 2016-07-14_10_14_01.lbv & 9 & 2 & Length & 50 & no & 5 \\
\hline 2016-07-14_11_18_01.lbv & 12 & 2 & Length & 50 & no & 5 \\
\hline 2016-07-14_10_14_02.1bv & 9 & 2 & Length & 50 & yes & 5 \\
\hline 2016-07-14_11_18_02.lbv & 12 & 2 & Length & 50 & yes & 5 \\
\hline $2016-07-14 \_10 \_57 \_01.1 b v$ & 9 & 2 & Length & 30 & no & 5 \\
\hline 2016-07-14_13_31_01.lbv & 12 & 2 & Length & 30 & no & 5 \\
\hline 2016-07-14_11_03_01.lbv & 9 & 2 & Length & 100 & no & 5 \\
\hline 2016-07-14_13_37_01.lbv & 12 & 2 & Length & 100 & no & 5 \\
\hline
\end{tabular}

Table A2. File names and test protocols for the second series of testing

\begin{tabular}{|c|c|c|c|}
\hline File name & Scan number & \begin{tabular}{|c|} 
Impulse duration, \\
half-periods
\end{tabular} & Orientation \\
\hline 2016-08-08_14_03_01.lbv & 1 & 1 & Height \\
\hline 2016-08-08_14_03_02.lbv & 2 & 1 & Height \\
\hline 2016-08-08_14_03_03.lbv & 3 & 1 & Height \\
\hline 2016-08-08_14_03_04.lbv & 4 & 1 & Height \\
\hline 2016-08-08_14_03_05.lbv & 5 & 1 & Height \\
\hline 2016-08-08_14_03_06.lbv & 6 & 1 & Height \\
\hline 2016-08-08_14_03_07.lbv & 7 & 1 & Height \\
\hline 2016-08-08_14_03_08.lbv & 8 & 1 & Height \\
\hline 2016-08-08_14_03_09.lbv & 9 & 1 & Height \\
\hline 2016-08-08_14_03_10.lbv & 10 & 1 & Height \\
\hline 2016-08-08_14_03_11.lbv & 11 & 1 & Height \\
\hline 2016-08-08_14_03_12.lbv & 12 & 1 & Height \\
\hline 2016-08-08_14_03_13.lbv & 13 & 1 & Height \\
\hline 2016-08-08_14_03_14.lbv & 14 & 1 & Height \\
\hline 2016-08-08_14_03_15.lbv & 15 & 1 & Height \\
\hline 2016-08-08_14_03_16.lbv & 16 & 1 & Height \\
\hline 2016-08-08_14_03_17.lbv & 17 & 1 & Height \\
\hline 2016-08-08_14_03_18.1bv & 18 & 1 & Height \\
\hline 2016-08-08_14_03_19.1bv & 19 & 1 & Height \\
\hline 2016-08-08_13_33_01.lbv & 1 & 2 & Height \\
\hline 2016-08-08_13_33_02.lbv & 2 & 2 & Height \\
\hline 2016-08-08_13_33_03.lbv & 3 & 2 & Height \\
\hline 2016-08-08_13_33_04.lbv & 4 & 2 & Height \\
\hline 2016-08-08_13_33_05.lbv & 5 & 2 & Height \\
\hline 2016-08-08_13_33_06.lbv & 6 & 2 & Height \\
\hline 2016-08-08_13_33_07.lbv & 7 & 2 & Height \\
\hline 2016-08-08_13_33_08.lbv & 8 & 2 & Height \\
\hline 2016-08-08_13_33_09.lbv & 9 & 2 & Height \\
\hline 2016-08-08_13_33_10.lbv & 10 & 2 & Height \\
\hline 2016-08-08_13_33_11.lbv & 11 & 2 & Height \\
\hline 2016-08-08_13_33_12.lbv & 12 & 2 & Height \\
\hline 2016-08-08_13_33_13.lbv & 13 & 2 & Height \\
\hline 2016-08-08_13_33_14.lbv & 14 & 2 & Height \\
\hline 2016-08-08_13_33_15.lbv & 15 & 2 & Height \\
\hline
\end{tabular}


Table A2. File names and test protocols for the second series of testing (continued)

\begin{tabular}{|c|c|c|c|}
\hline File name & Scan number & $\begin{array}{c}\text { Impulse duration, } \\
\text { half-periods }\end{array}$ & Orientation \\
\hline 2016-08-08_13_33_16.lbv & 16 & 2 & Height \\
\hline 2016-08-08_13_33_17.lbv & 17 & 2 & Height \\
\hline 2016-08-08_13_33_18.1bv & 18 & 2 & Height \\
\hline 2016-08-08_13_33_19.1bv & 19 & 2 & Height \\
\hline 2016-08-08_14_26_01.lbv & 1 & 4 & Height \\
\hline 2016-08-08_14_26_02.lbv & 2 & 4 & Height \\
\hline 2016-08-08_14_26_03.1bv & 3 & 4 & Height \\
\hline 2016-08-08_14_26_04.lbv & 4 & 4 & Height \\
\hline 2016-08-08_14_26_05.lbv & 5 & 4 & Height \\
\hline 2016-08-08_14_26_06.lbv & 6 & 4 & Height \\
\hline 2016-08-08_14_26_07.lbv & 7 & 4 & Height \\
\hline 2016-08-08_14_26_08.lbv & 8 & 4 & Height \\
\hline 2016-08-08_14_26_09.lbv & 9 & 4 & Height \\
\hline 2016-08-08_14_26_10.lbv & 10 & 4 & Height \\
\hline 2016-08-08_14_26_11.lbv & 11 & 4 & Height \\
\hline 2016-08-08_14_26_12.lbv & 12 & 4 & Height \\
\hline 2016-08-08_14_26_13.lbv & 13 & 4 & Height \\
\hline 2016-08-08_14_26_14.lbv & 14 & 4 & Height \\
\hline 2016-08-08_14_26_15.lbv & 15 & 4 & Height \\
\hline 2016-08-08_14_26_16.lbv & 16 & 4 & Height \\
\hline 2016-08-08_14_26_17.lbv & 17 & 4 & Height \\
\hline 2016-08-08_14_26_18.lbv & 18 & 4 & Height \\
\hline 2016-08-08_14_26_19.lbv & 19 & 4 & Height \\
\hline 2016-08-08_51_26_01.lbv & 1 & 16 & Height \\
\hline 2016-08-08_51_26_02.lbv & 2 & 16 & Height \\
\hline 2016-08-08_51_26_03.lbv & 3 & 16 & Height \\
\hline 2016-08-08_51_26_04.lbv & 4 & 16 & Height \\
\hline 2016-08-08_51_26_05.lbv & 5 & 16 & Height \\
\hline 2016-08-08_51_26_06.lbv & 6 & 16 & Height \\
\hline 2016-08-08_51_26_07.lbv & 7 & 16 & Height \\
\hline 2016-08-08_51_26_08.lbv & 8 & 16 & Height \\
\hline 2016-08-08_51_26_09.lbv & 9 & 16 & Height \\
\hline 2016-08-08_51_26_10.1bv & 10 & 16 & Height \\
\hline 2016-08-08_51_26_11.lbv & 11 & 16 & Height \\
\hline 2016-08-08_51_26_12.lbv & 12 & 16 & Height \\
\hline 2016-08-08_51_26_13.lbv & 13 & 16 & Height \\
\hline 2016-08-08_51_26_14.lbv & 14 & 16 & Height \\
\hline 2016-08-08_51_26_15.lbv & 15 & 16 & Height \\
\hline 2016-08-08_51_26_16.lbv & 16 & 16 & Height \\
\hline 2016-08-08_51_26_17.lbv & 17 & 16 & Height \\
\hline 2016-08-08_51_26_18.lbv & 18 & 16 & Height \\
\hline 2016-08-08_51_26_19.1bv & 19 & 16 & Height \\
\hline 2016-08-08_10_30_07.lbv & 1 & 1 & Length \\
\hline 2016-08-08_10_30_06.lbv & 2 & 1 & Length \\
\hline 2016-08-08_10_30_05.lbv & 3 & 1 & Length \\
\hline 2016-08-08_10_30_04.lbv & 4 & 1 & Length \\
\hline 2016-08-08_10_30_03.lbv & 5 & 1 & Length \\
\hline 2016-08-08_10_30_03.lbv & 6 & 1 & Length \\
\hline 2016-08-08_10_30_01.lbv & 7 & 1 & Length \\
\hline
\end{tabular}


Table A2. File names and test protocols for the second series of testing (continued)

\begin{tabular}{|c|c|c|c|}
\hline File name & Scan number & $\begin{array}{c}\text { Impulse duration, } \\
\text { half-periods }\end{array}$ & Orientation \\
\hline 2016-08-08_10_17_07.lbv & 8 & 1 & Length \\
\hline 2016-08-08_10_17_06.lbv & 9 & 1 & Length \\
\hline 2016-08-08_10_17_05.lbv & 10 & 1 & Length \\
\hline 2016-08-08_10_17_04.lbv & 11 & 1 & Length \\
\hline 2016-08-08_10_17_03.lbv & 12 & 1 & Length \\
\hline 2016-08-08_10_17_02.lbv & 13 & 1 & Length \\
\hline 2016-08-08_10_17_01.lbv & 14 & 1 & Length \\
\hline 2016-08-08_10_05_05.lbv & 15 & 1 & Length \\
\hline 2016-08-08_10_05_04.lbv & 16 & 1 & Length \\
\hline 2016-08-08_10_05_03.lbv & 17 & 1 & Length \\
\hline 2016-08-08_10_05_02.lbv & 18 & 1 & Length \\
\hline 2016-08-08_10_05_01.lbv & 19 & 1 & Length \\
\hline 2016-08-08_09_31_21.lbv & 1 & 2 & Length \\
\hline 2016-08-08_09_31_20.lbv & 2 & 2 & Length \\
\hline 2016-08-08_09_31_19.lbv & 3 & 2 & Length \\
\hline 2016-08-08_09_31_18.lbv & 4 & 2 & Length \\
\hline 2016-08-08_09_31_17.lbv & 5 & 2 & Length \\
\hline 2016-08-08_09_31_16.lbv & 6 & 2 & Length \\
\hline 2016-08-08_09_31_15.lbv & 7 & 2 & Length \\
\hline 2016-08-08_09_31_14.lbv & 8 & 2 & Length \\
\hline 2016-08-08_09_31_11.lbv & 9 & 2 & Length \\
\hline 2016-08-08_09_31_10.lbv & 10 & 2 & Length \\
\hline 2016-08-08_09_31_09.lbv & 11 & 2 & Length \\
\hline 2016-08-08_09_31_08.lbv & 12 & 2 & Length \\
\hline 2016-08-08_09_31_07.lbv & 13 & 2 & Length \\
\hline 2016-08-08_09_31_06.lbv & 14 & 2 & Length \\
\hline 2016-08-08_09_31_05.lbv & 15 & 2 & Length \\
\hline 2016-08-08_09_31_04.lbv & 16 & 2 & Length \\
\hline 2016-08-08_09_31_03.lbv & 17 & 2 & Length \\
\hline 2016-08-08_09_31_02.lbv & 18 & 2 & Length \\
\hline 2016-08-08_09_31_01.lbv & 19 & 2 & Length \\
\hline 2016-08-08_10_41_19.lbv & 1 & 4 & Length \\
\hline 2016-08-08_10_41_18.lbv & 2 & 4 & Length \\
\hline 2016-08-08_10_41_17.lbv & 3 & 4 & Length \\
\hline 2016-08-08_10_41_16.lbv & 4 & 4 & Length \\
\hline 2016-08-08_10_41_15.lbv & 5 & 4 & Length \\
\hline 2016-08-08_10_41_14.lbv & 6 & 4 & Length \\
\hline 2016-08-08_10_41_13.lbv & 7 & 4 & Length \\
\hline 2016-08-08_10_41_12.lbv & 8 & 4 & Length \\
\hline 2016-08-08_10_41_11.lbv & 9 & 4 & Length \\
\hline 2016-08-08_10_41_10.lbv & 10 & 4 & Length \\
\hline 2016-08-08_10_41_09.lbv & 11 & 4 & Length \\
\hline 2016-08-08_10_41_08.lbv & 12 & 4 & Length \\
\hline 2016-08-08_10_41_07.lbv & 13 & 4 & Length \\
\hline 2016-08-08_10_41_06.lbv & 14 & 4 & Length \\
\hline 2016-08-08_10_41_05.lbv & 15 & 4 & Length \\
\hline 2016-08-08_10_41_04.lbv & 16 & 4 & Length \\
\hline 2016-08-08_10_41_03.lbv & 17 & 4 & Length \\
\hline 2016-08-08_10_41_02.lbv & 18 & 4 & Length \\
\hline
\end{tabular}


Table A2. File names and test protocols for the second series of testing (continued)

\begin{tabular}{|c|c|c|c|}
\hline File name & Scan number & \begin{tabular}{|c|} 
Impulse duration, \\
half-periods
\end{tabular} & Orientation \\
\hline 2016-08-08_10_41_01.lbv & 19 & 4 & Length \\
\hline 2016-08-08_11_04_19.1bv & 1 & 16 & Length \\
\hline 2016-08-08_11_04_18.1bv & 2 & 16 & Length \\
\hline 2016-08-08_11_04_17.lbv & 3 & 16 & Length \\
\hline 2016-08-08_11_04_16.lbv & 4 & 16 & Length \\
\hline 2016-08-08_11_04_15.lbv & 5 & 16 & Length \\
\hline 2016-08-08_11_04_14.lbv & 6 & 16 & Length \\
\hline 2016-08-08_11_04_13.lbv & 7 & 16 & Length \\
\hline 2016-08-08_11_04_12.lbv & 8 & 16 & Length \\
\hline 2016-08-08_11_04_11.lbv & 9 & 16 & Length \\
\hline 2016-08-08_11_04_10.1bv & 10 & 16 & Length \\
\hline 2016-08-08_11_04_09.1bv & 11 & 16 & Length \\
\hline 2016-08-08_11_04_08.1bv & 12 & 16 & Length \\
\hline 2016-08-08_11_04_07.lbv & 13 & 16 & Length \\
\hline 2016-08-08_11_04_06.lbv & 14 & 16 & Length \\
\hline 2016-08-08_11_04_05.lbv & 15 & 16 & Length \\
\hline 2016-08-08_11_04_04.lbv & 16 & 16 & Length \\
\hline 2016-08-08_11_04_03.lbv & 17 & 16 & Length \\
\hline 2016-08-08_11_04_02.lbv & 18 & 16 & Length \\
\hline 2016-08-08_11_04_01.lbv & 19 & 16 & Length \\
\hline
\end{tabular}




\section{APPENDIX B. PANORAMIC IMAGES FOR HEIGHT ORIENTATION}





\section{APPENDIX B. PANORAMIC IMAGES FOR HEIGHT ORIENTATION}

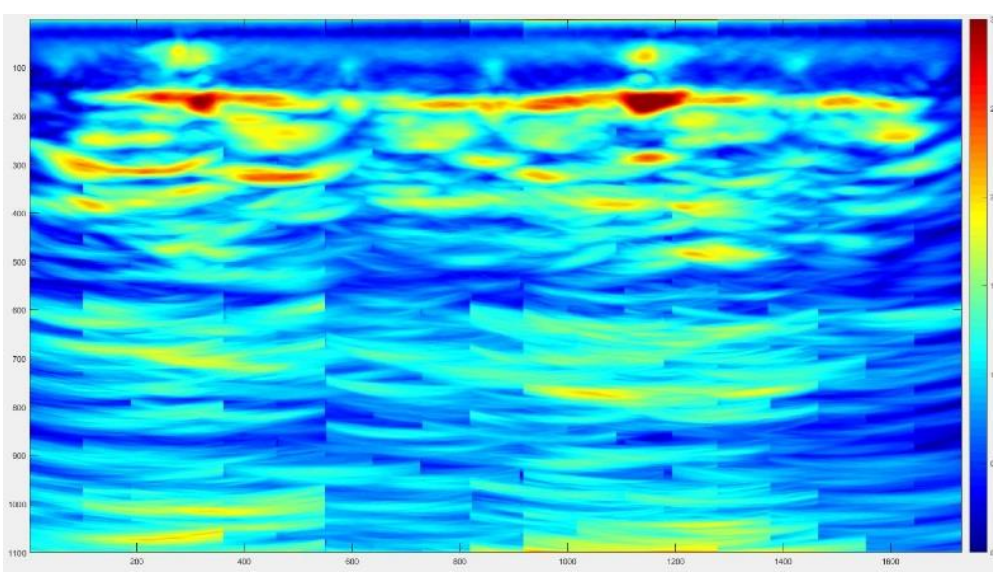

One half-period duration of the emitted signal

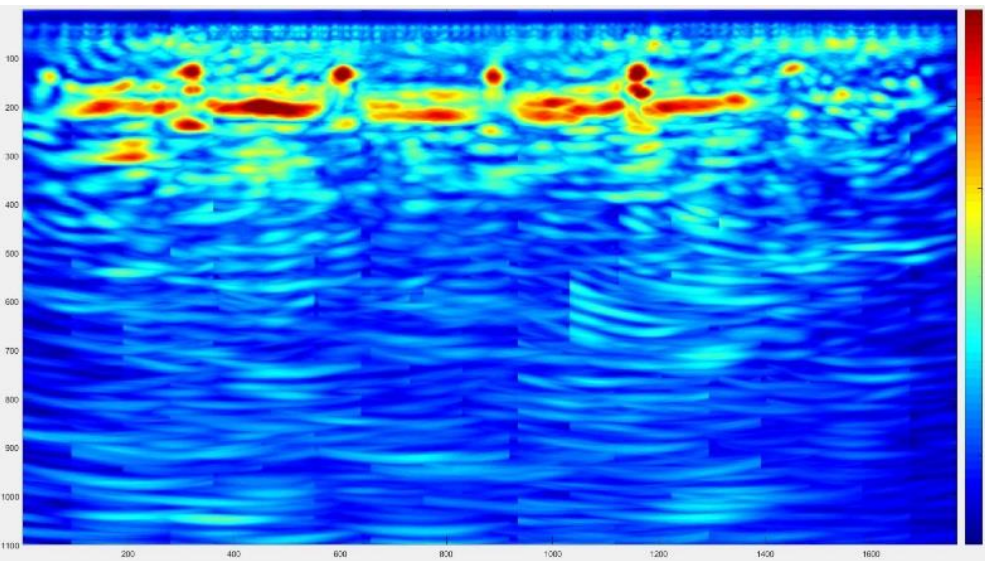

Four half-periods duration of the emitted signal

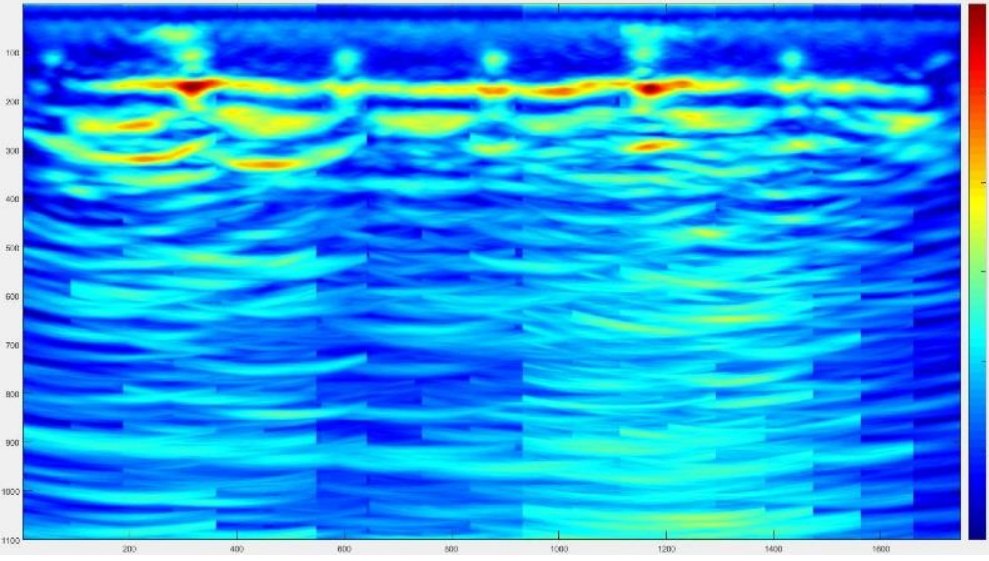

Two half-periods duration of the emitted signal

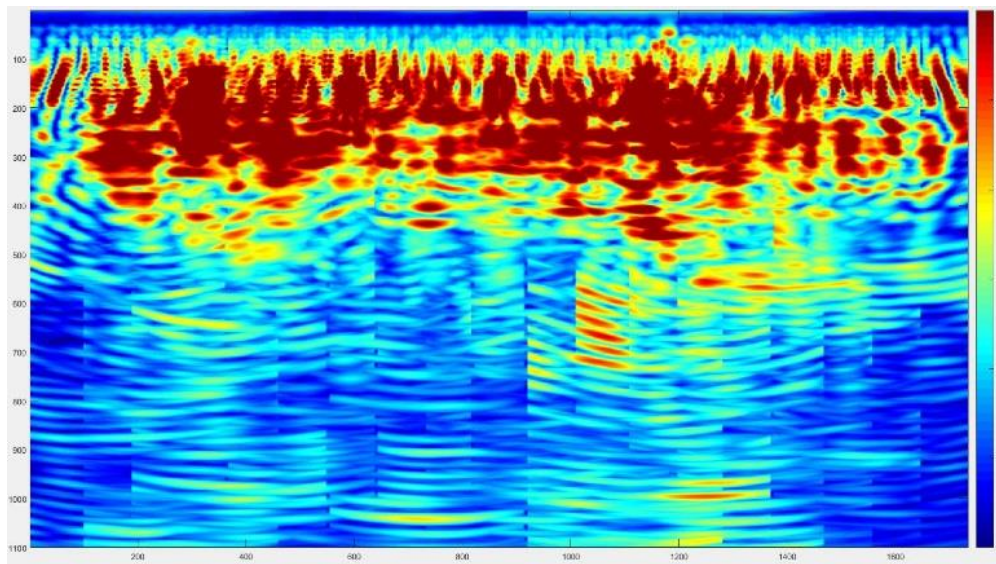

Sixteen half-periods duration of the emitted signal

Panoramic B-scan, row 1. 


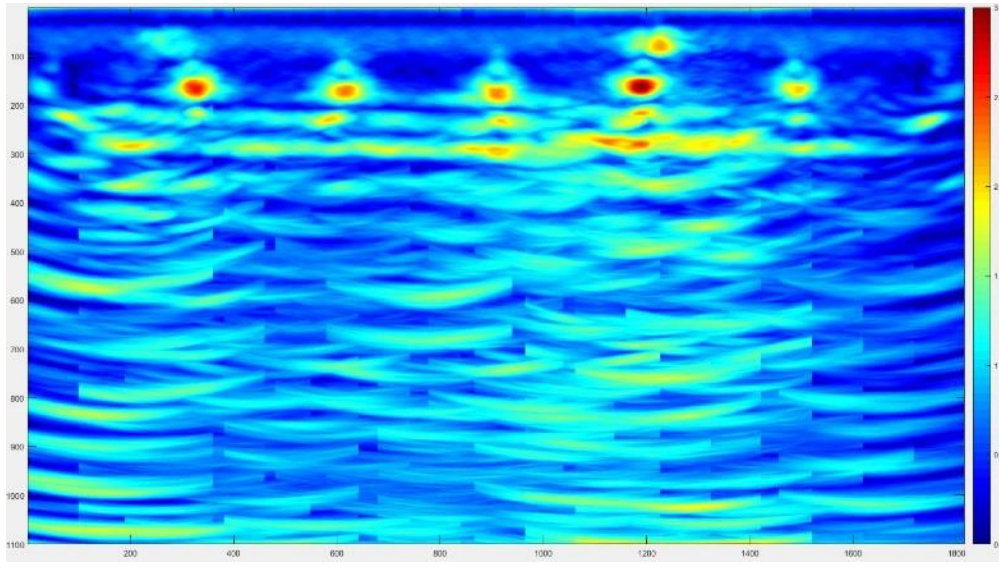

One half-period duration of the emitted signal

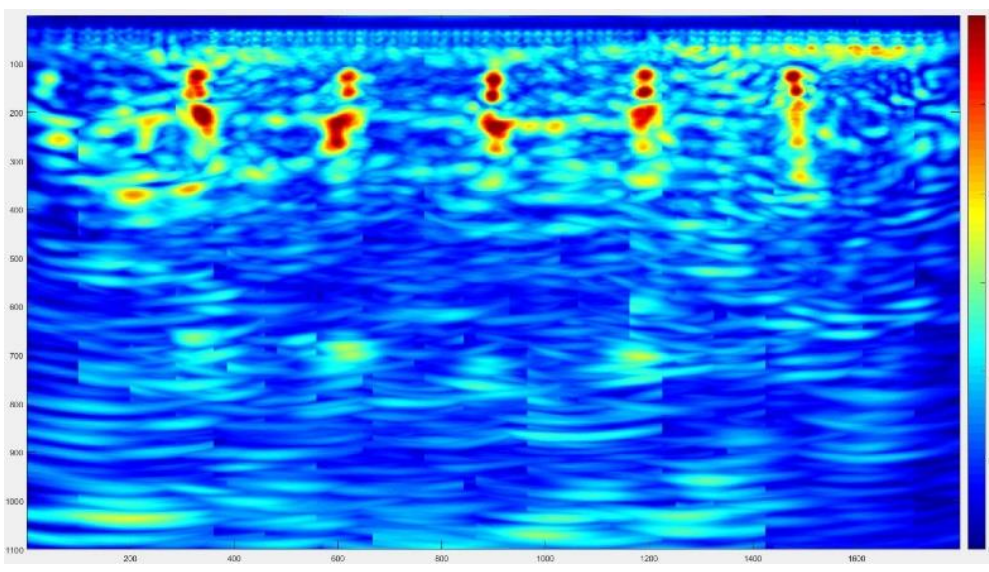

Four half-periods duration of the emitted signal

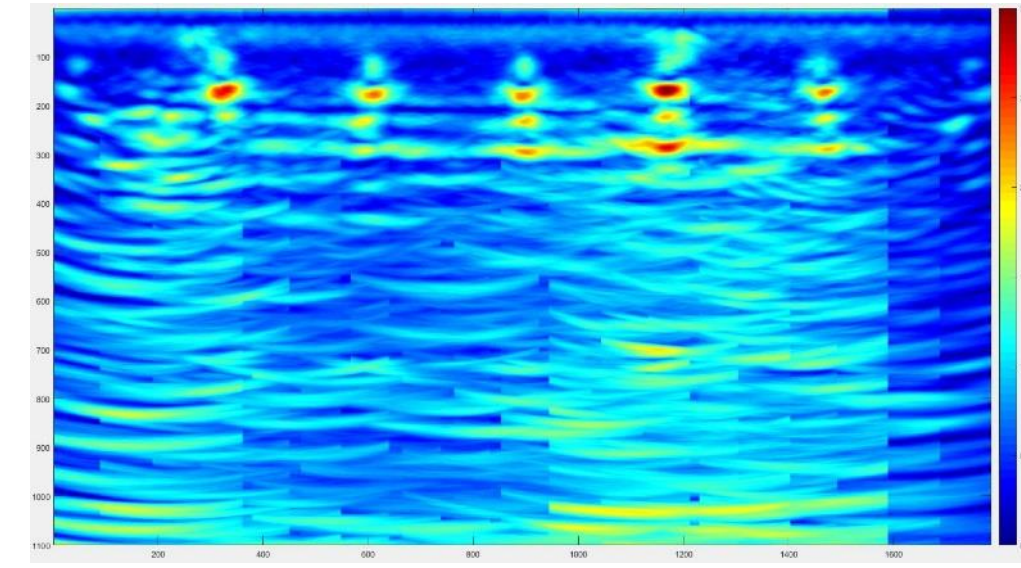

Two half-periods duration of the emitted signal

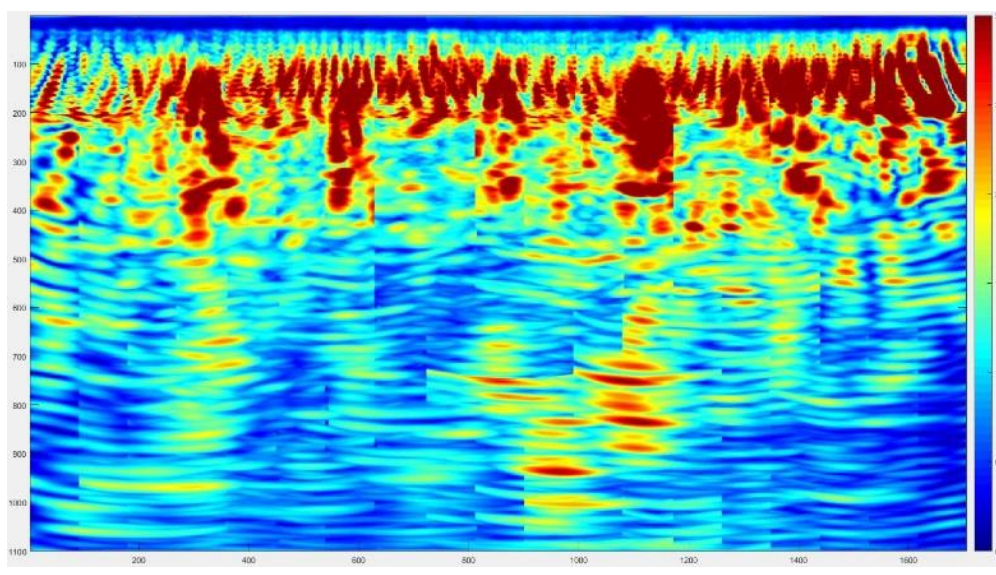

Sixteen half-periods duration of the emitted signal

Panoramic B-scan, row 2. 


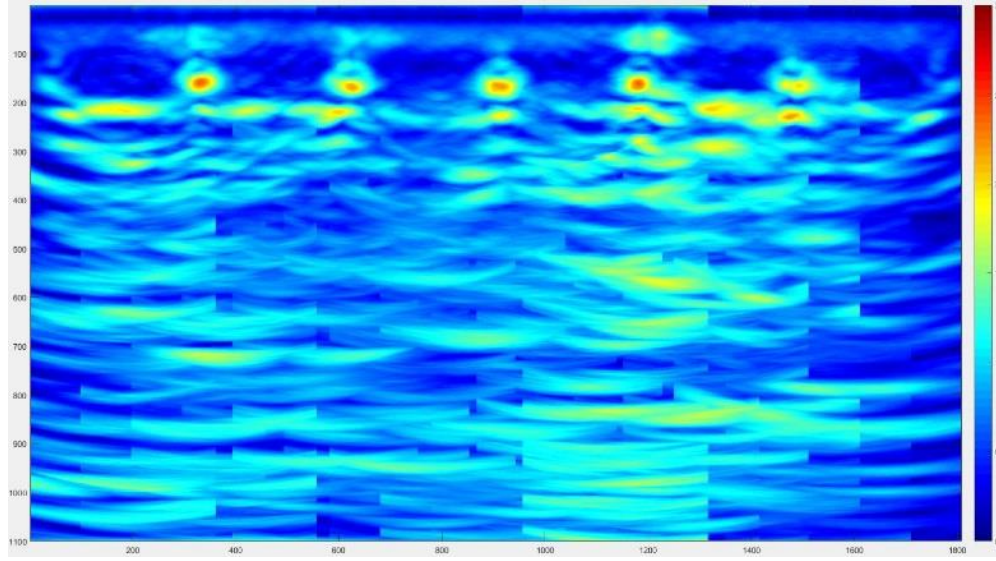

One half-period duration of the emitted signal

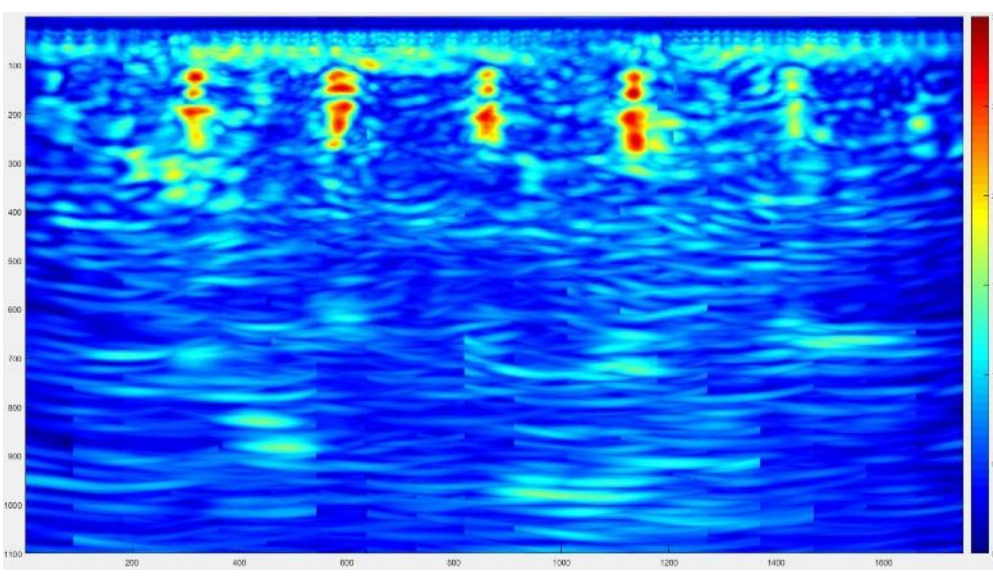

Four half-periods duration of the emitted signal

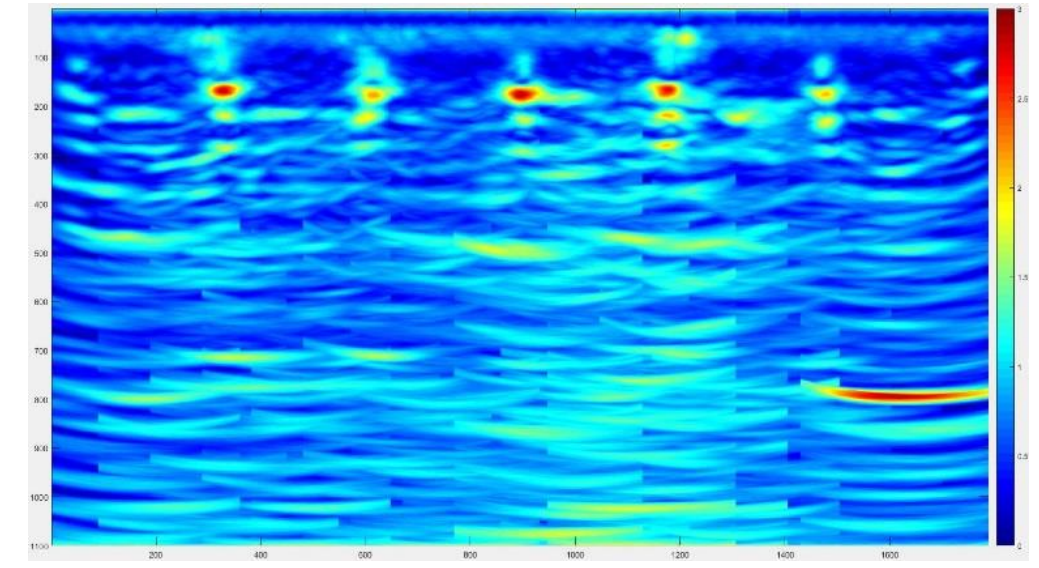

Two half-periods duration of the emitted signal

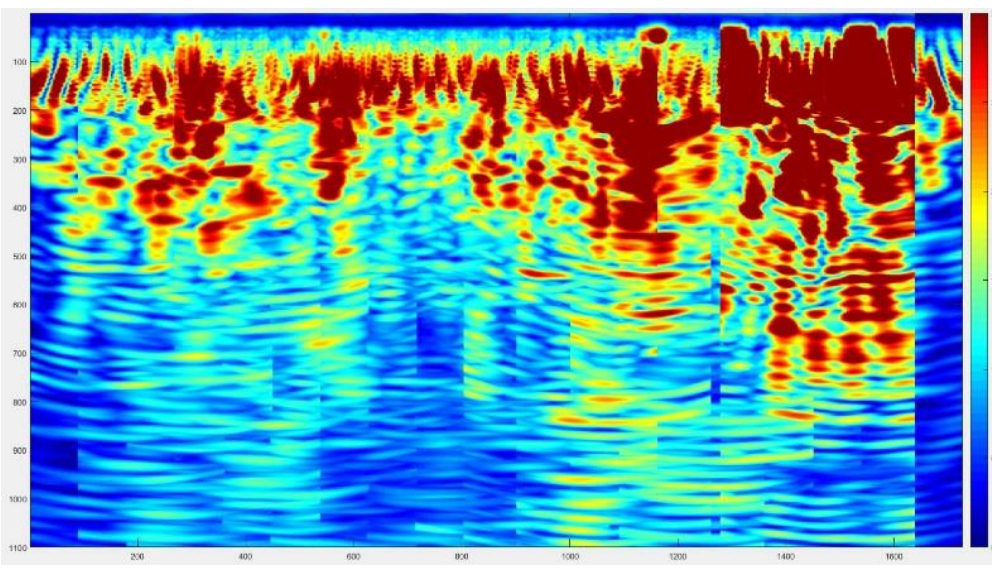

Sixteen half-periods duration of the emitted signal

Panoramic B-scan, row 3. 


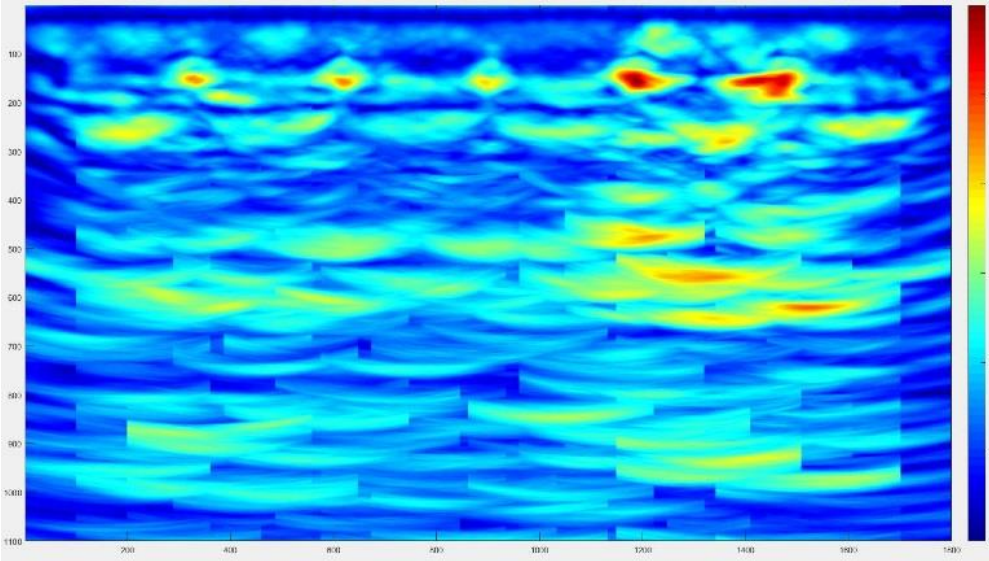

One half-period duration of the emitted signal

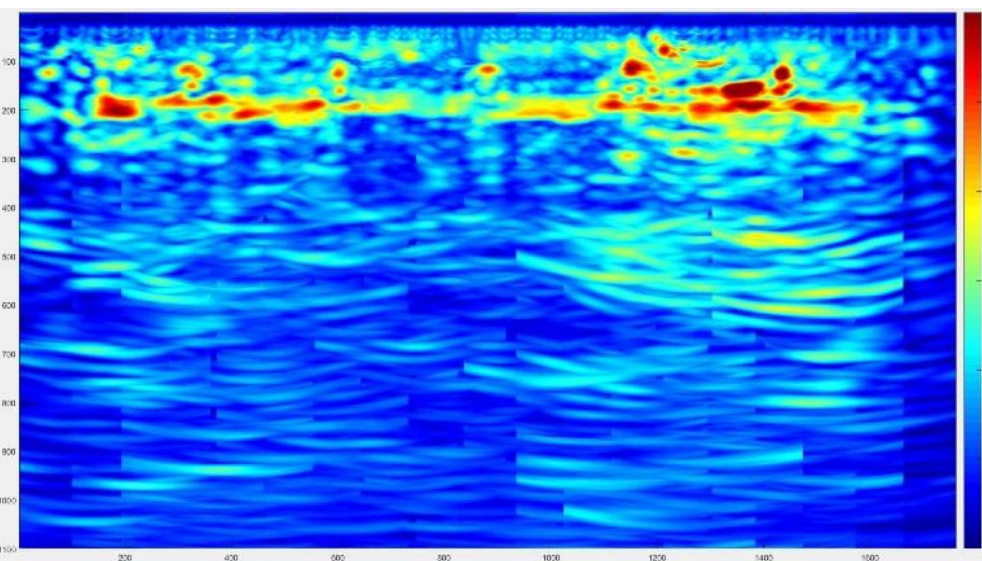

Four half-periods duration of the emitted signal

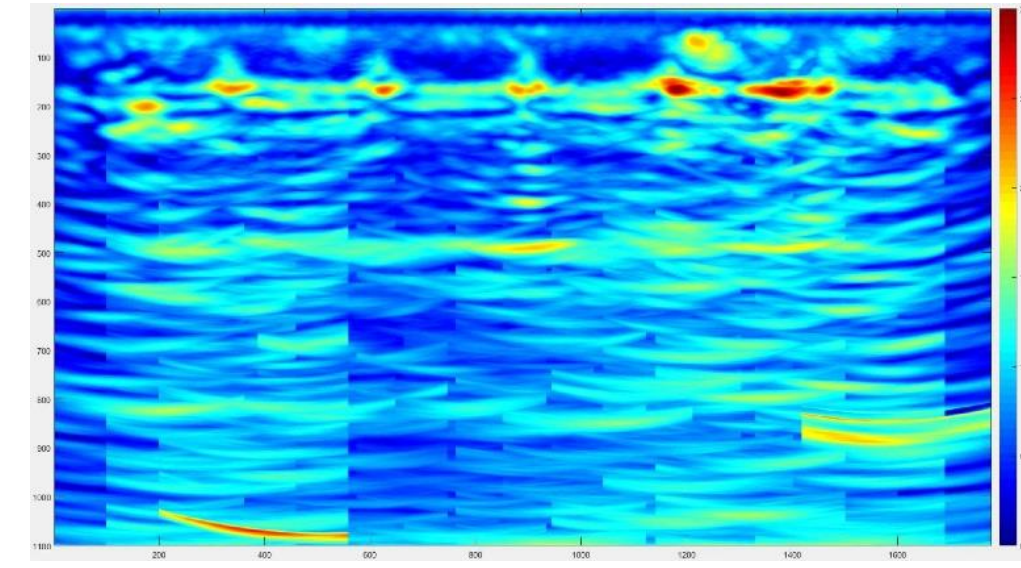

Two half-periods duration of the emitted signal

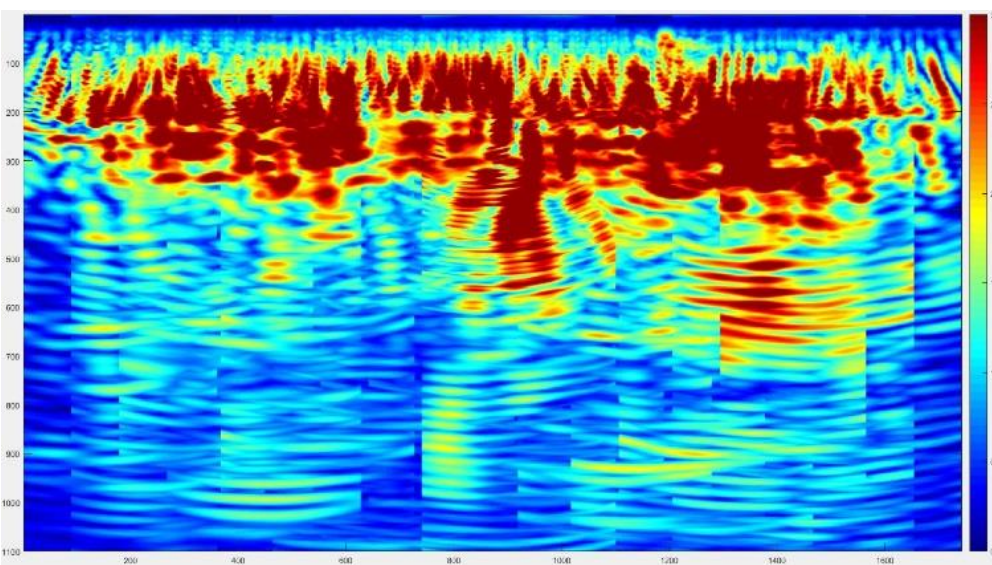

Sixteen half-periods duration of the emitted signal

Panoramic B-scan, row 4. 


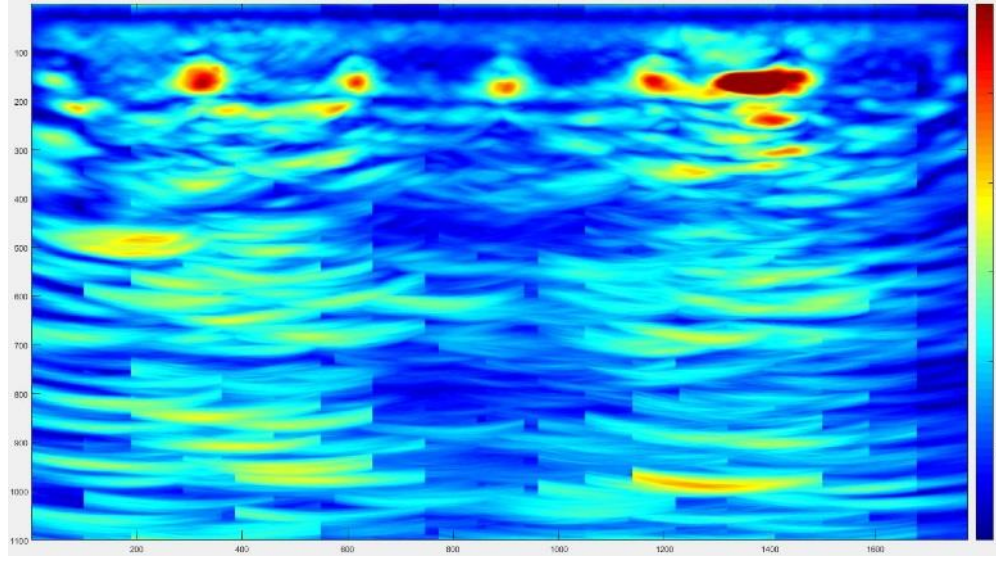

One half-period duration of the emitted signal

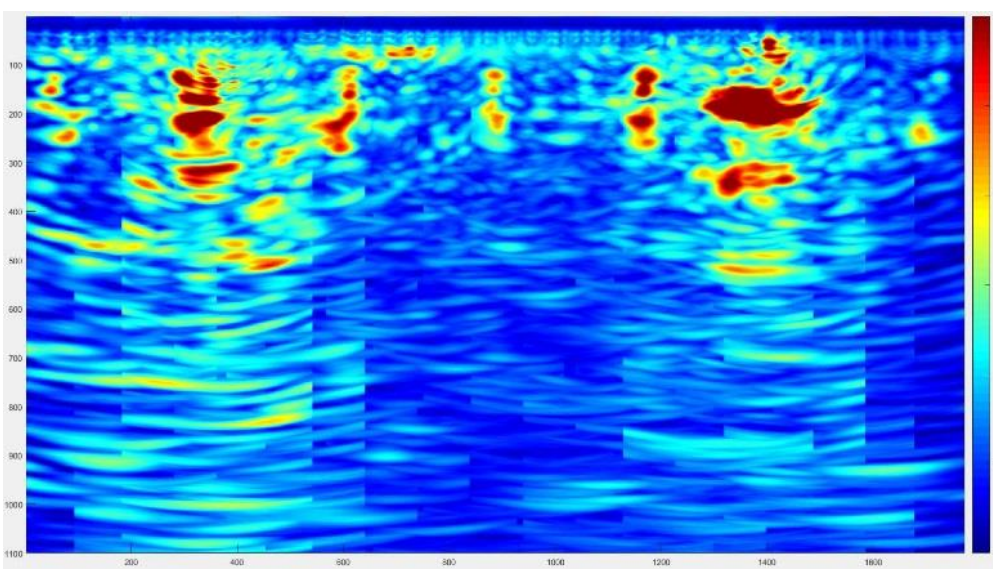

Four half-periods duration of the emitted signal

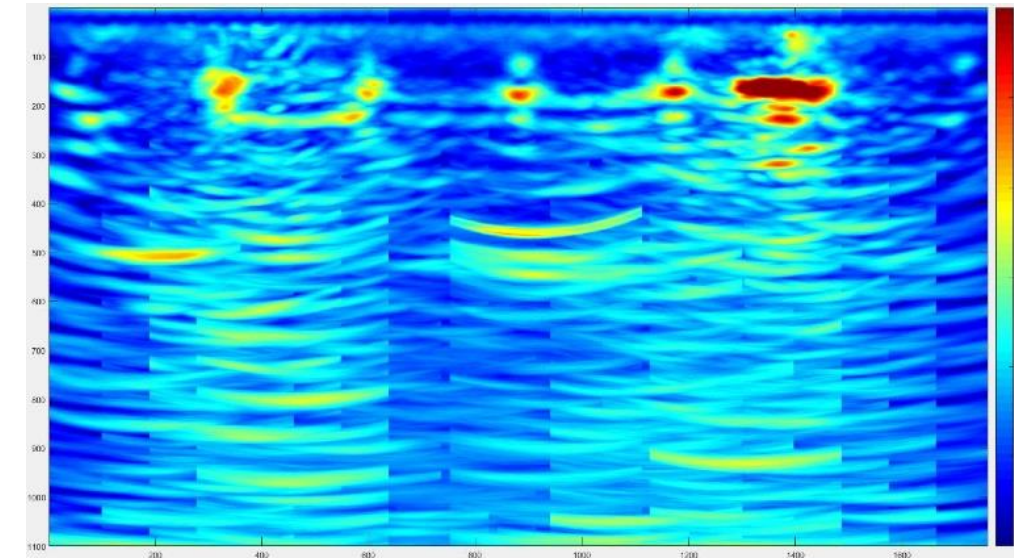

Two half-periods duration of the emitted signal

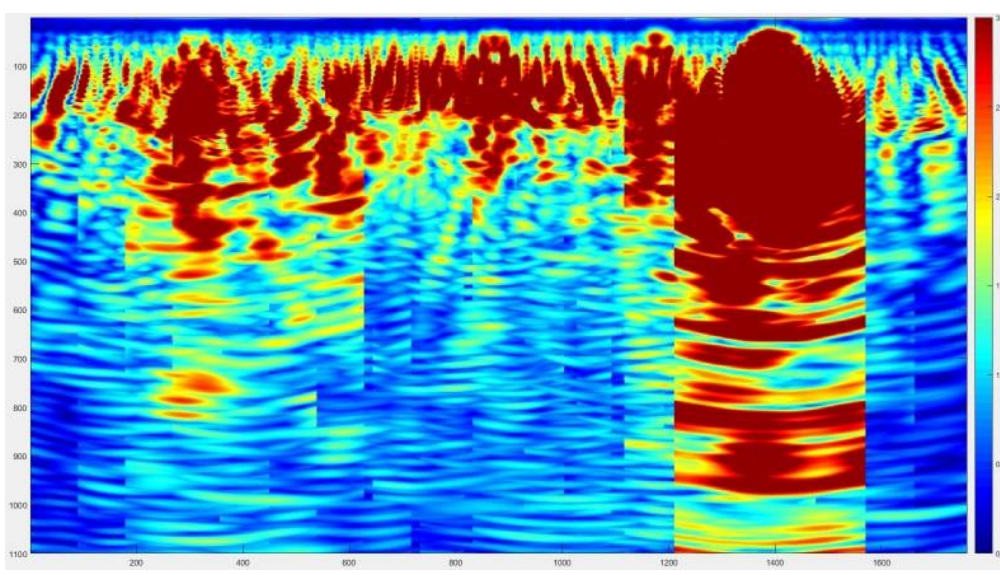

Sixteen half-periods duration of the emitted signal

Panoramic B-scan, row 5. 


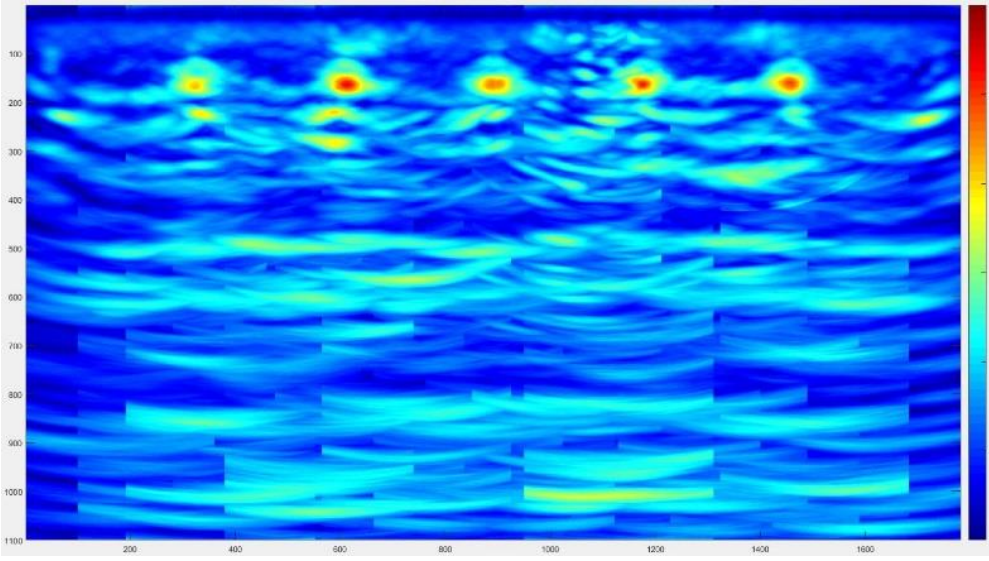

One half-period duration of the emitted signal

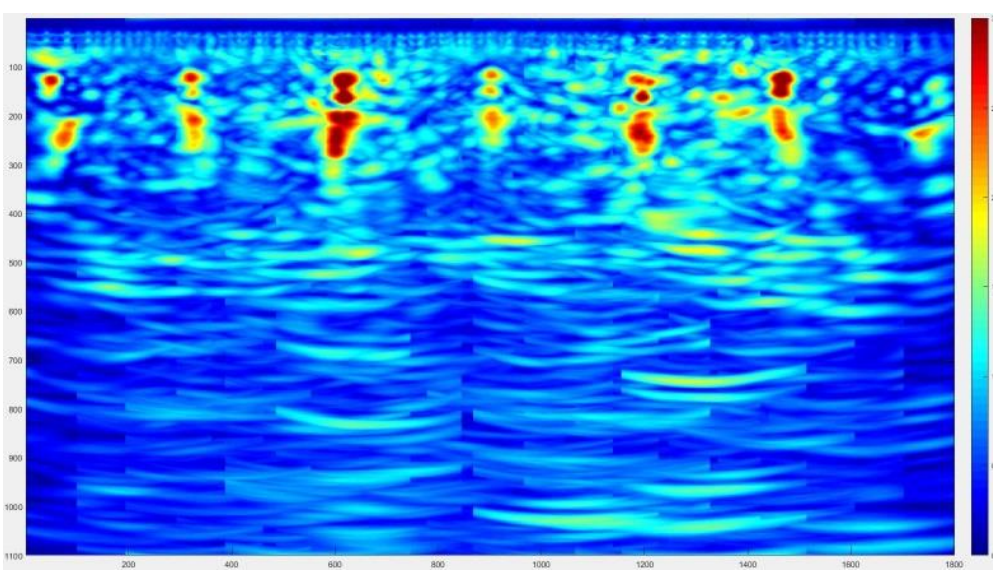

Four half-periods duration of the emitted signal

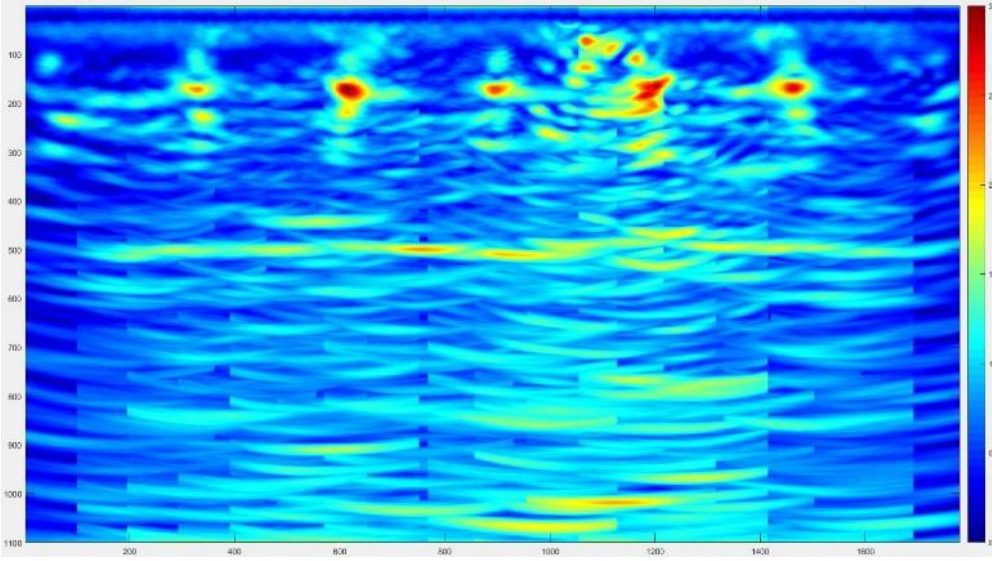

Two half-periods duration of the emitted signal

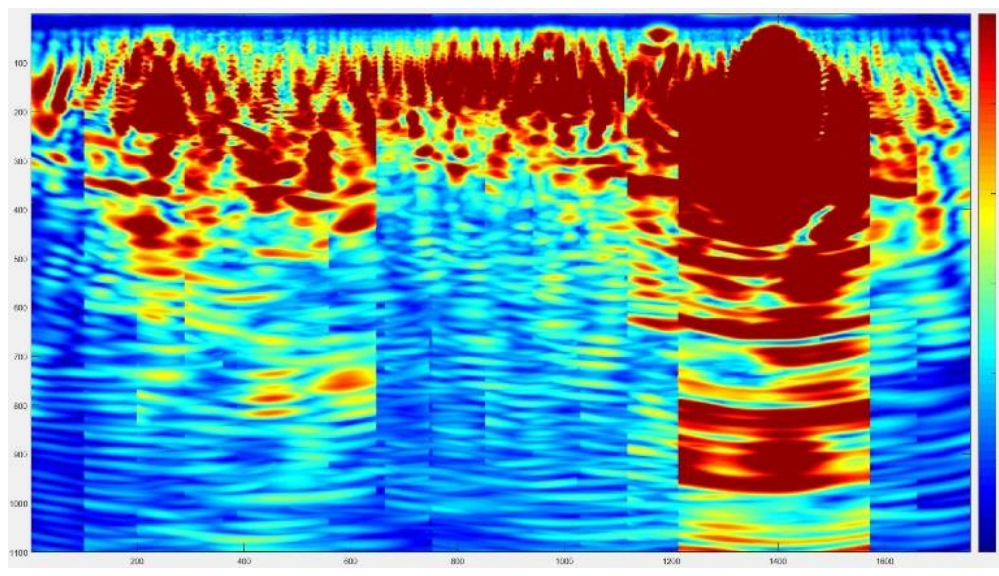

Sixteen half-periods duration of the emitted signal

Panoramic B-scan, row 6. 


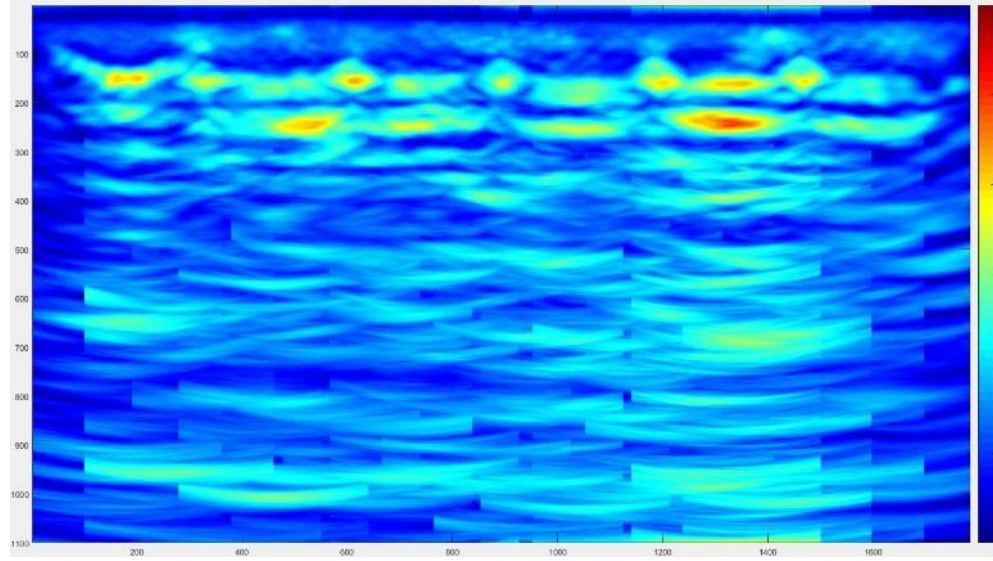

One half-period duration of the emitted signal

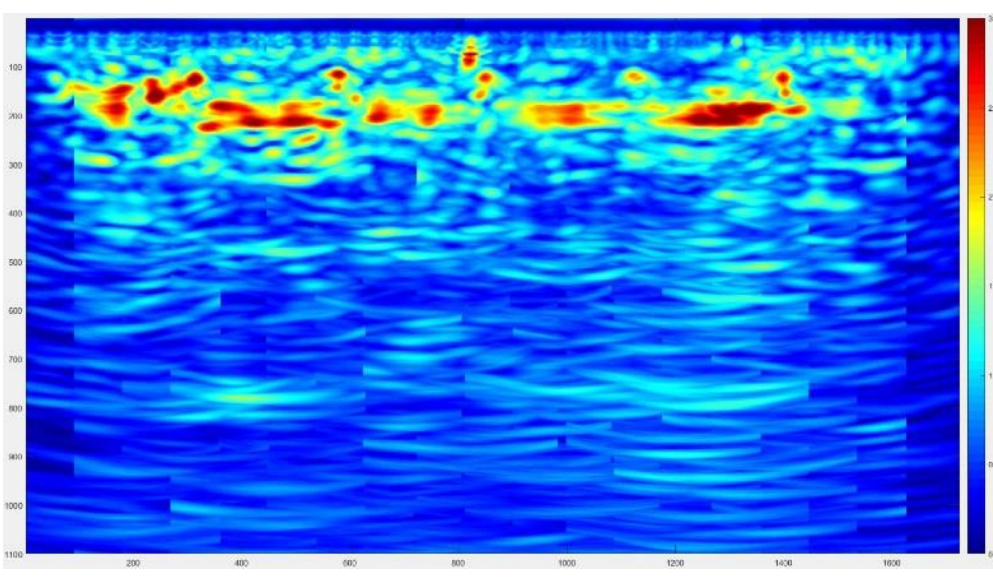

Four half-periods duration of the emitted signal

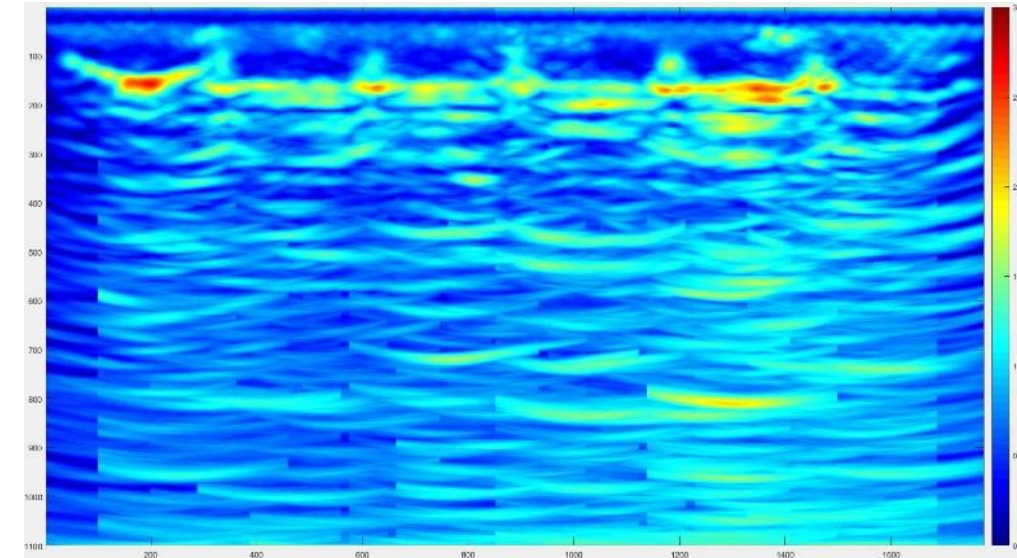

Two half-periods duration of the emitted signal

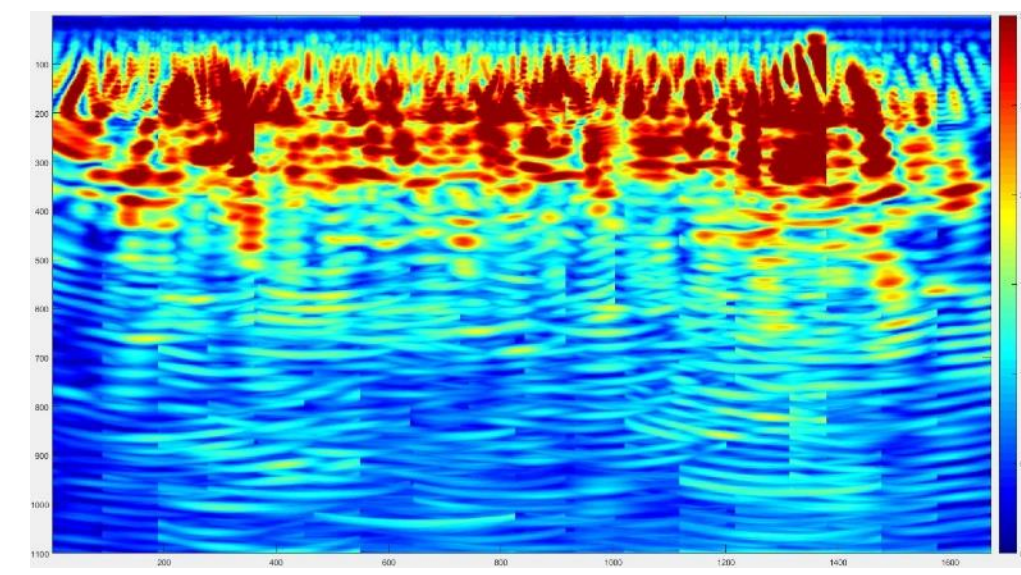

Sixteen half-periods duration of the emitted signal

Panoramic B-scan, row 7. 


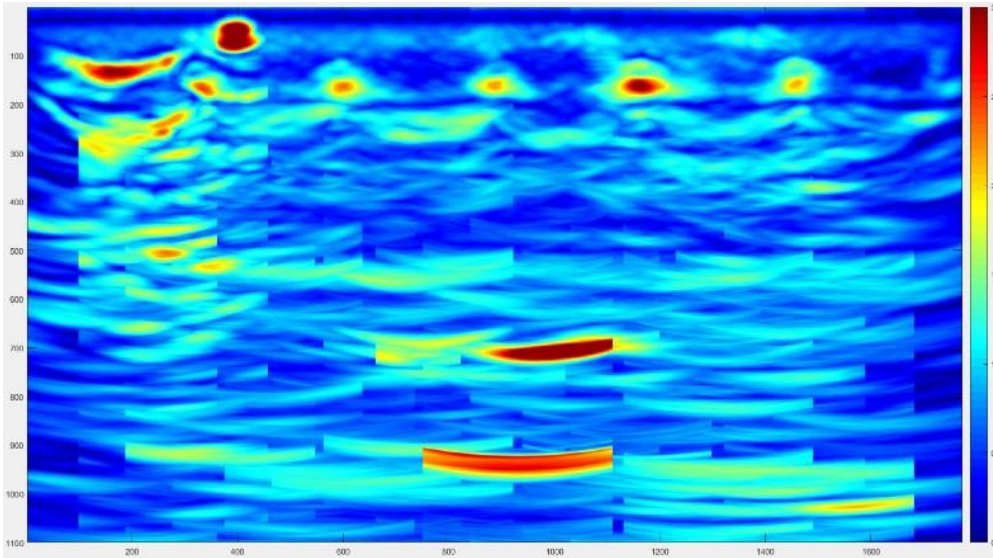

One half-period duration of the emitted signal

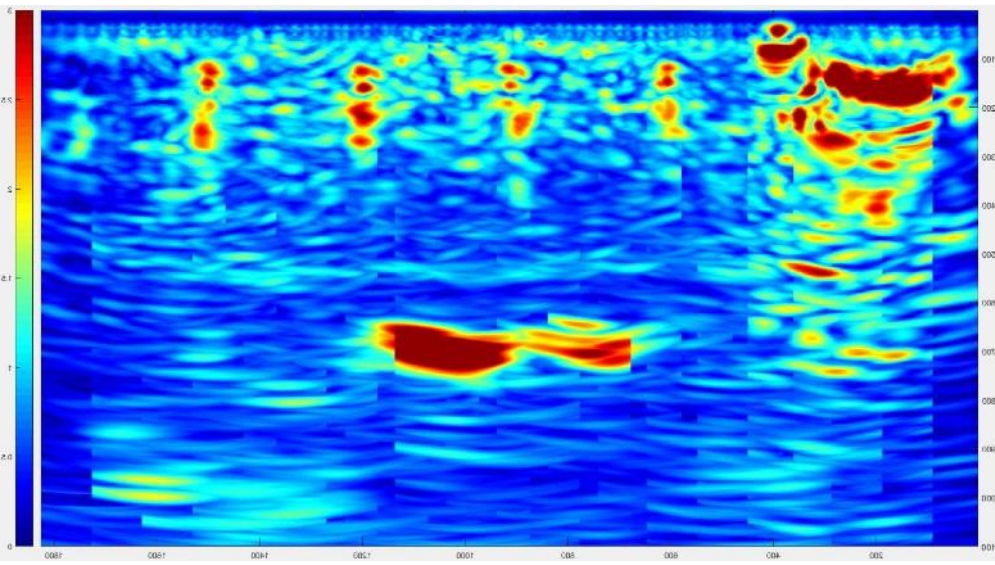

Four half-periods duration of the emitted signal

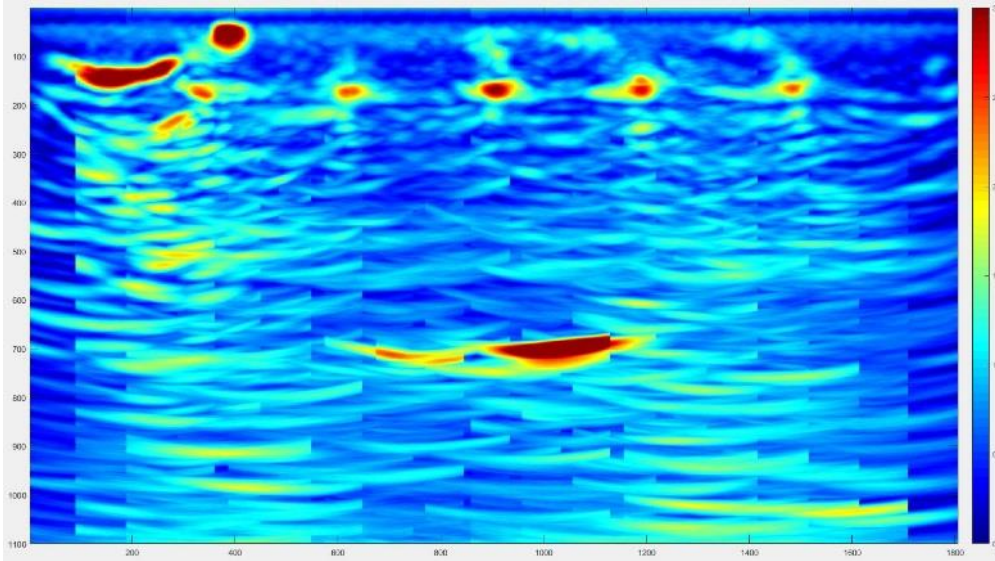

Two half-periods duration of the emitted signal

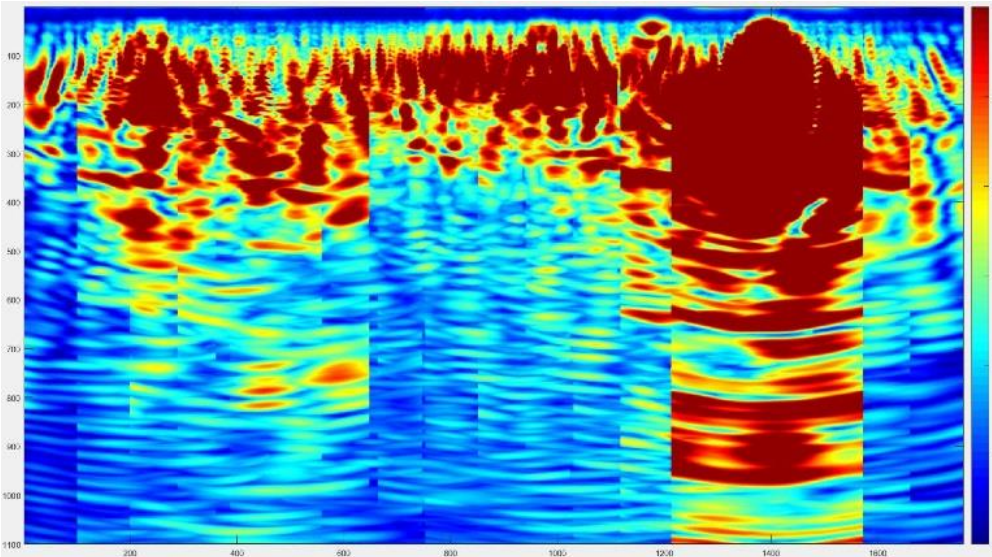

Sixteen half-periods duration of the emitted signal

Panoramic B-scan, row 8 


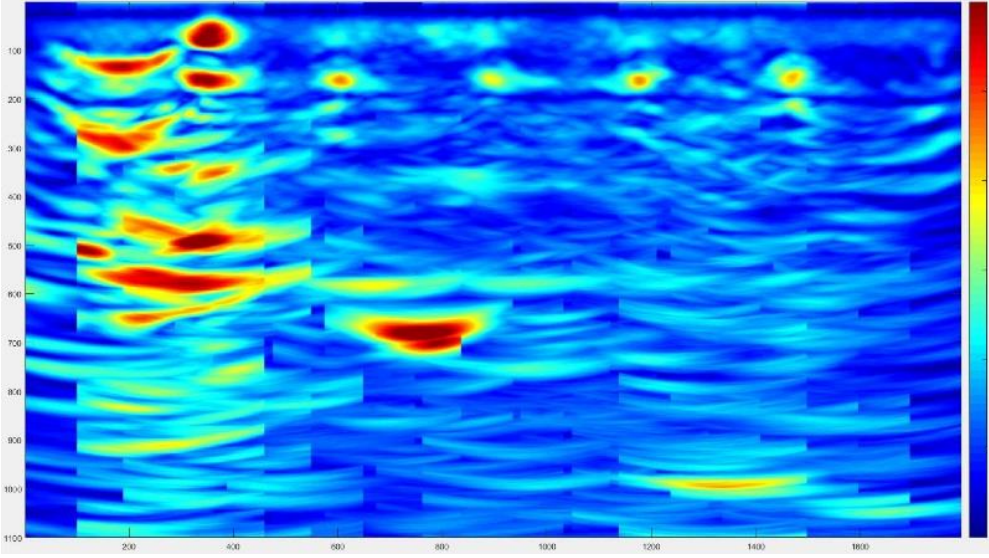

One half-period duration of the emitted signal

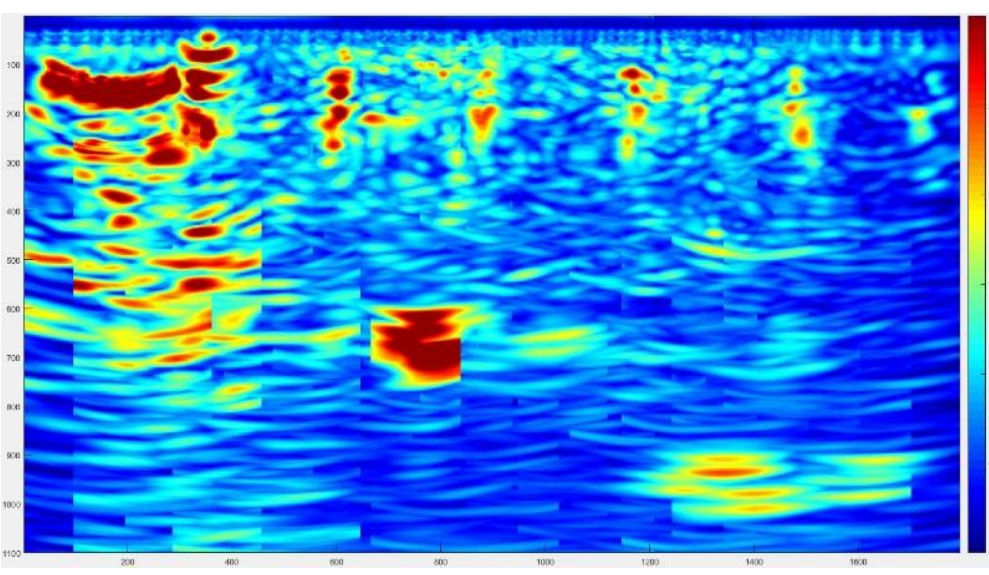

Four half-periods duration of the emitted signal

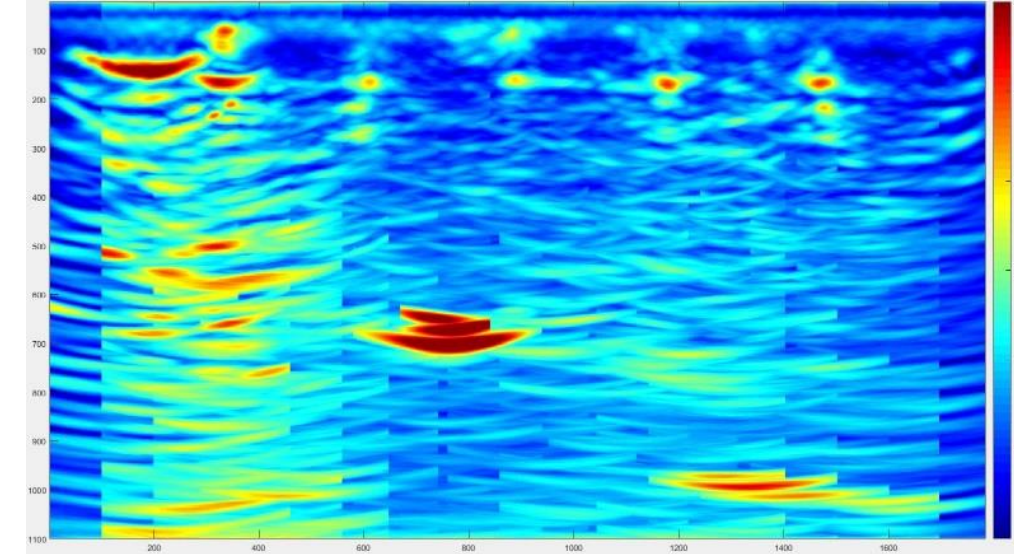

Two half-periods duration of the emitted signal

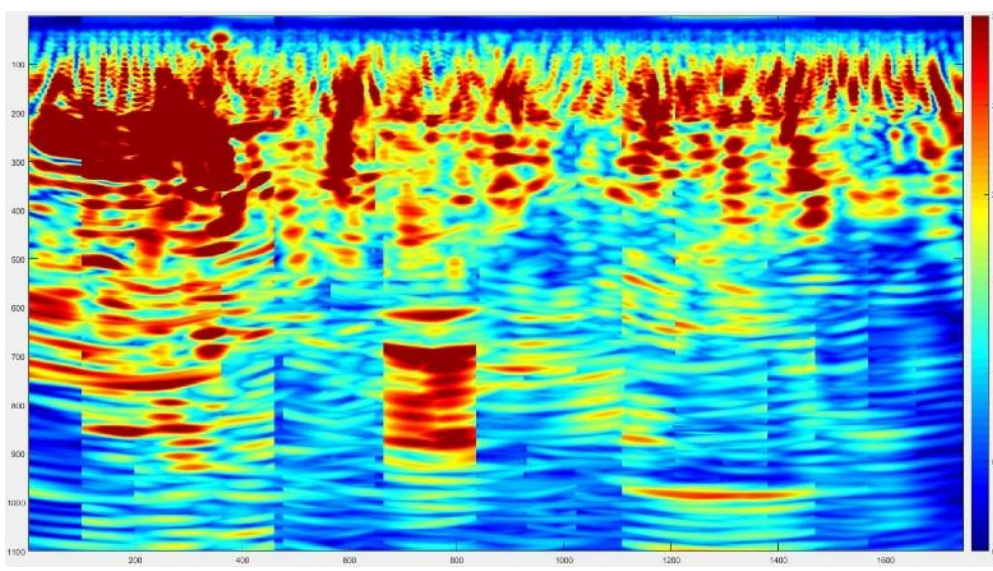

Sixteen half-periods duration of the emitted signal

Panoramic B-scan, row 9. 


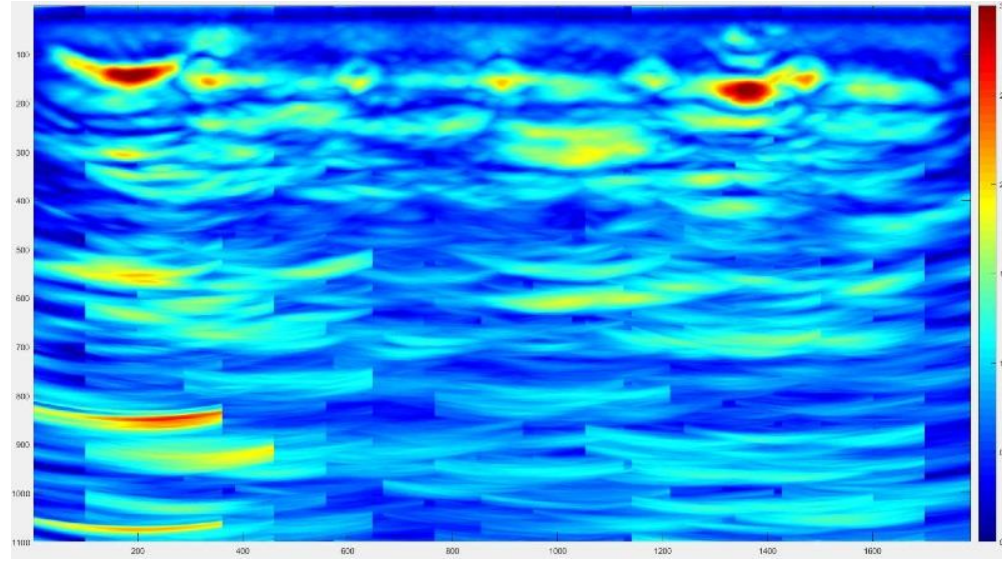

One half-period duration of the emitted signal

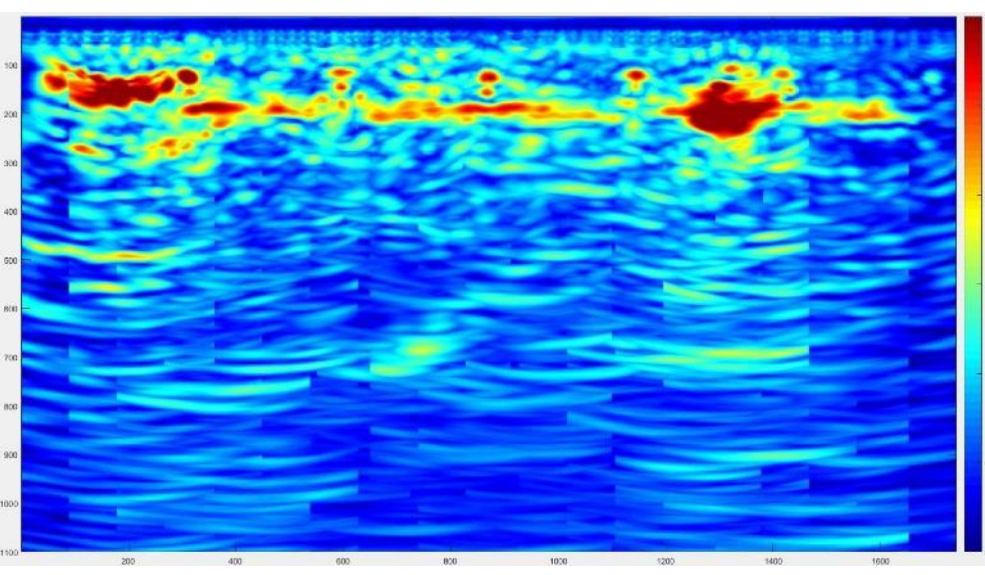

Four half-periods duration of the emitted signal

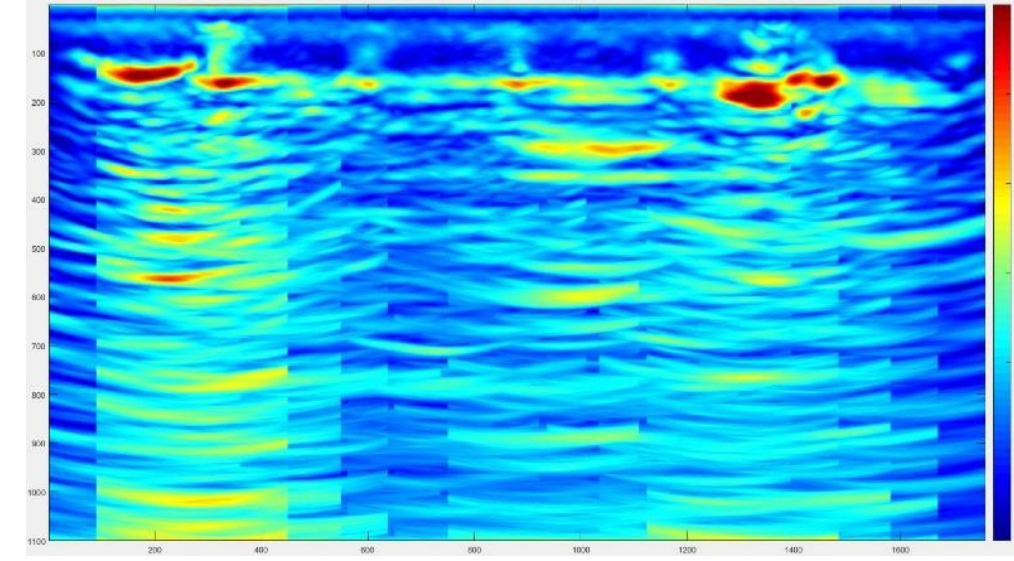

Two half-periods duration of the emitted signal

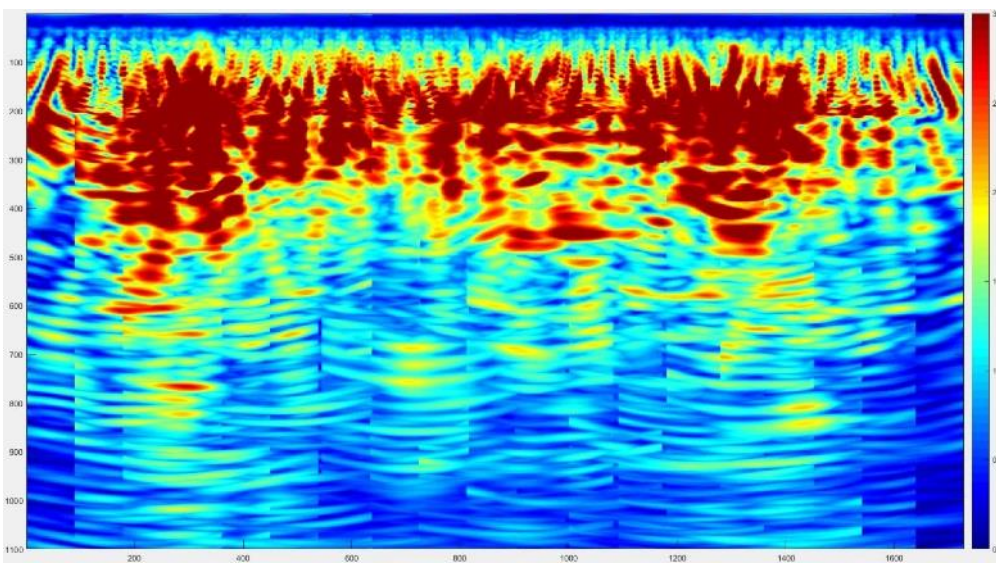

Sixteen half-periods duration of the emitted signal

Panoramic B-scan, row 10. 


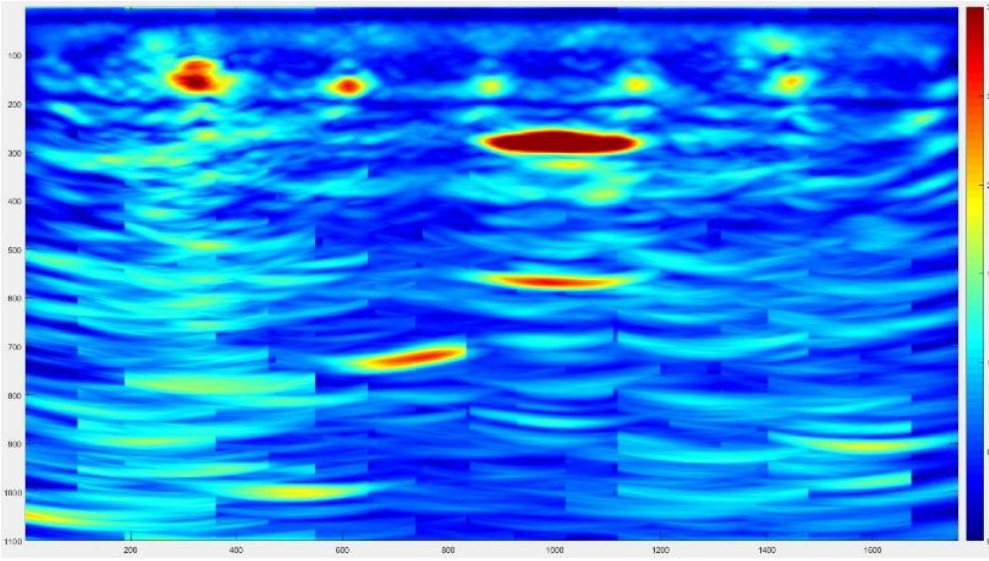

One half-period duration of the emitted signal

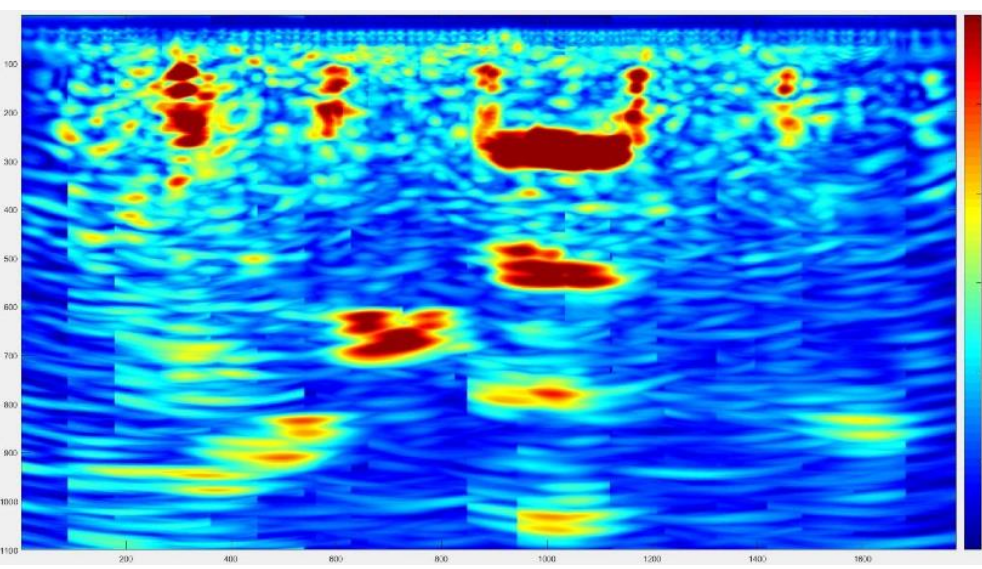

Four half-periods duration of the emitted signal

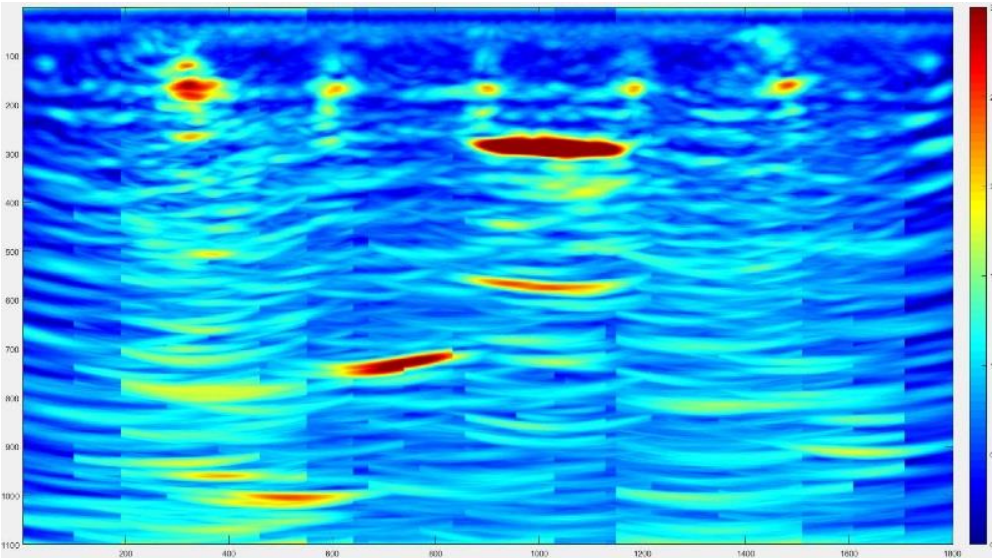

Two half-periods duration of the emitted signal

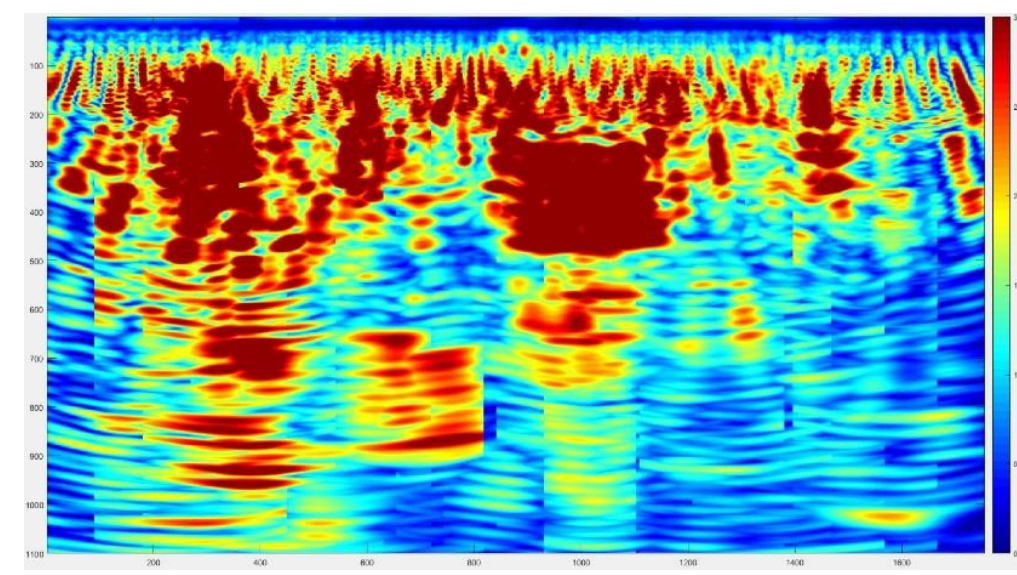

Sixteen half-periods duration of the emitted signal

Panoramic B-scan, row 11. 


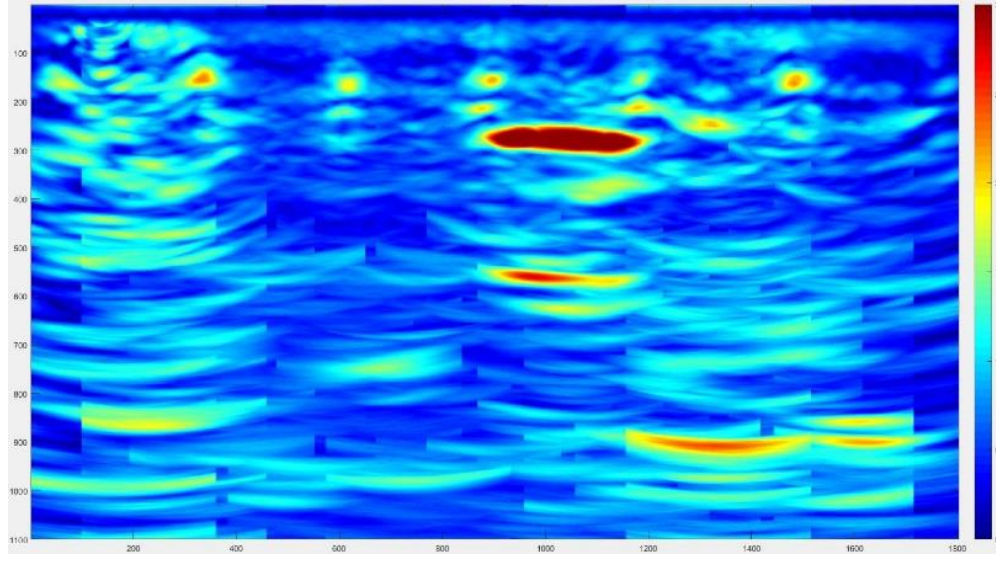

One half-period duration of the emitted signal

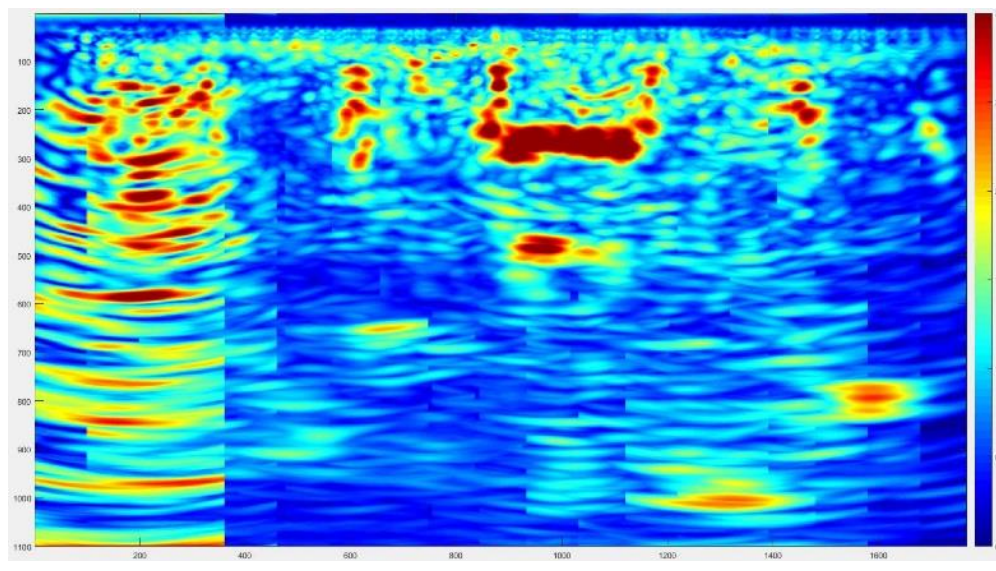

Four half-periods duration of the emitted signal

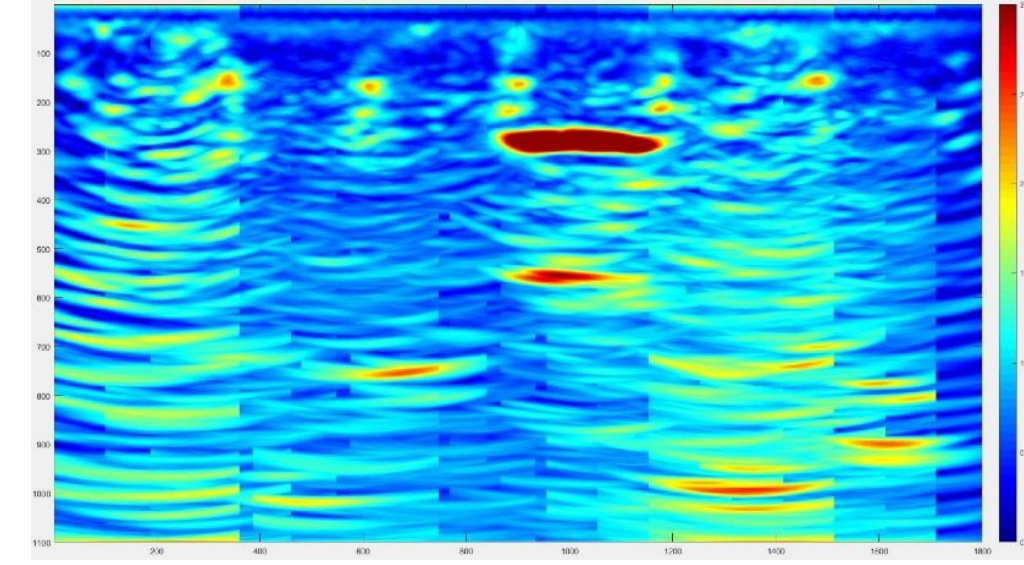

Two half-periods duration of the emitted signal

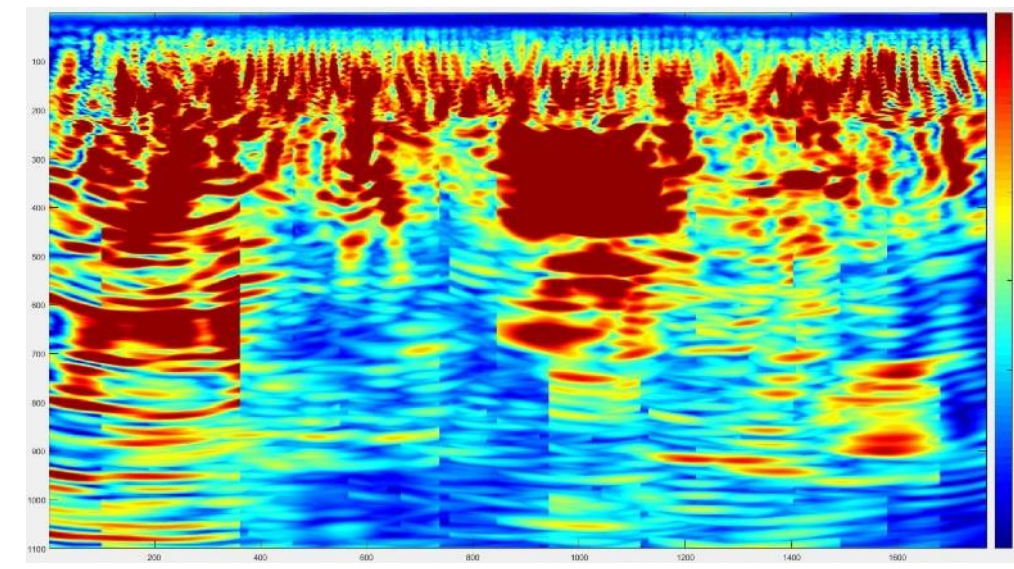

Sixteen half-periods duration of the emitted signal

Panoramic B-scan, row 12. 


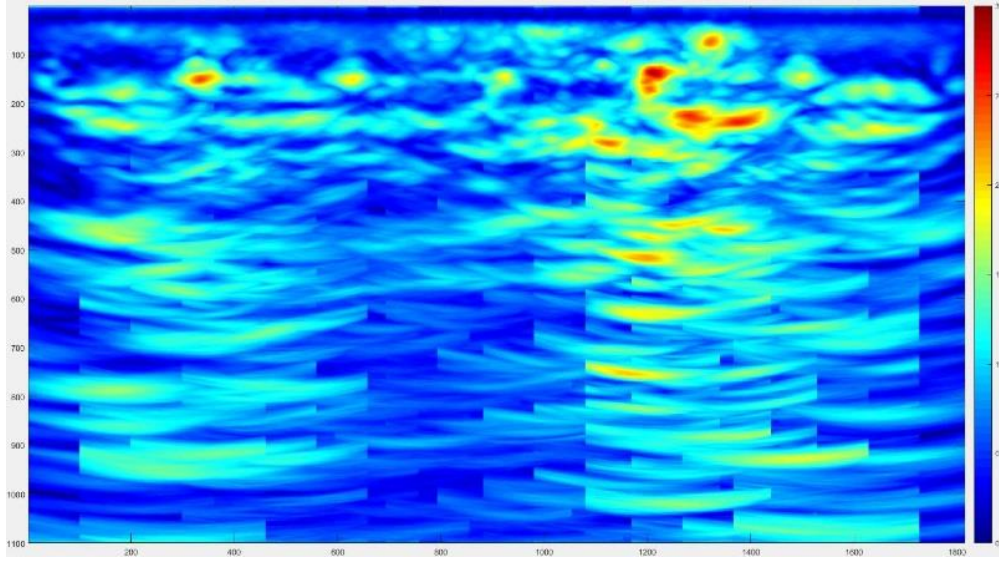

One half-period duration of the emitted signal

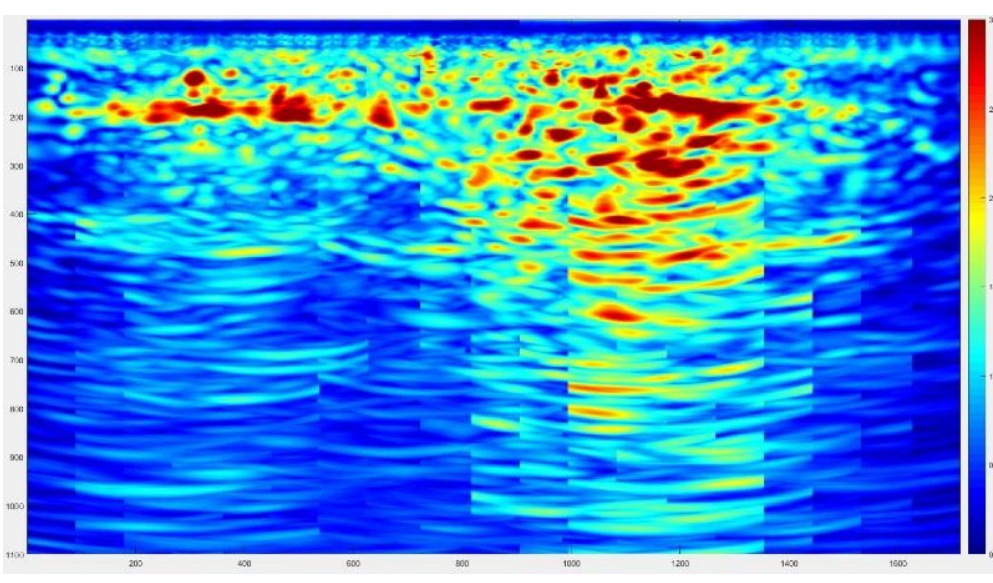

Four half-periods duration of the emitted signal

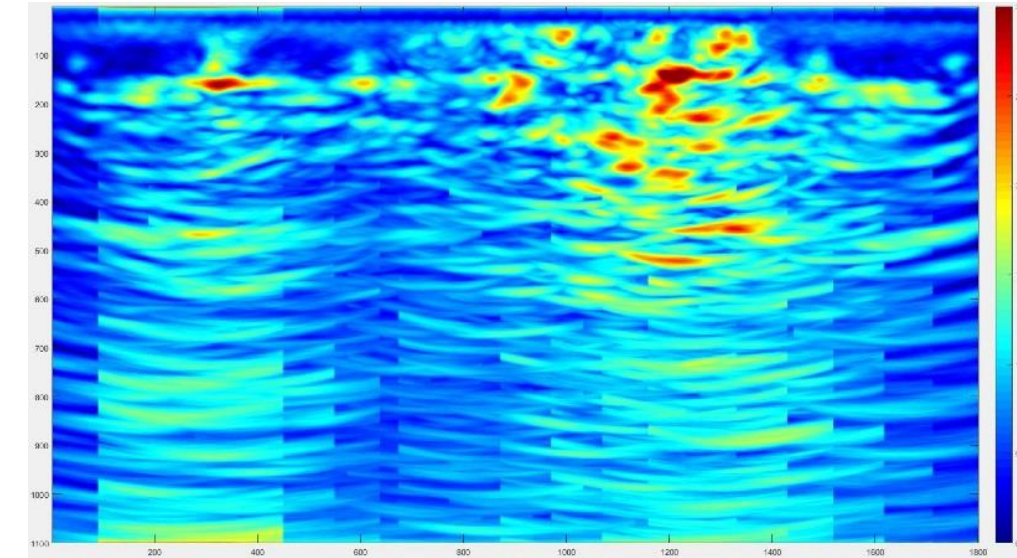

Two half-periods duration of the emitted signal

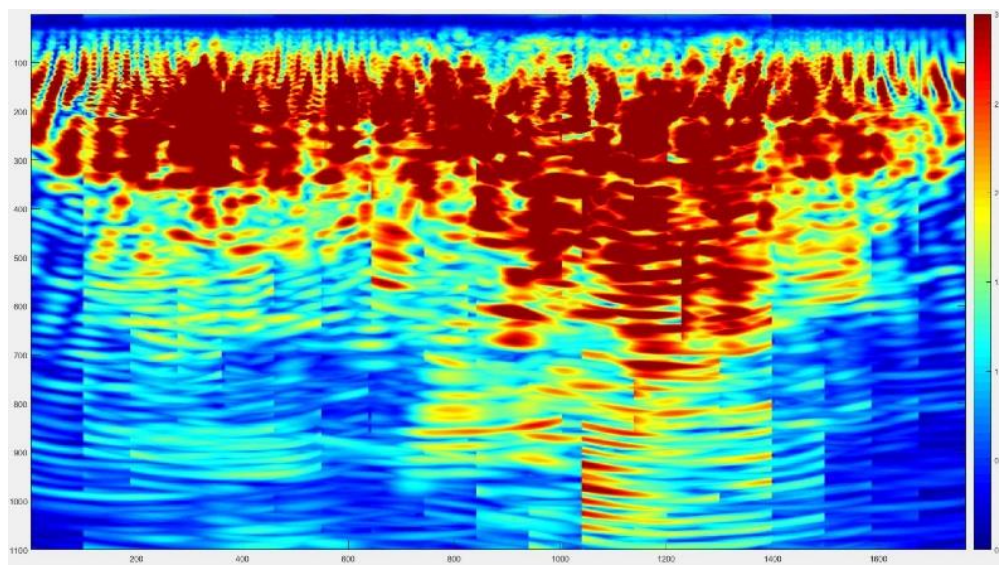

Sixteen half-periods duration of the emitted signal

Panoramic B-scan, row 13. 


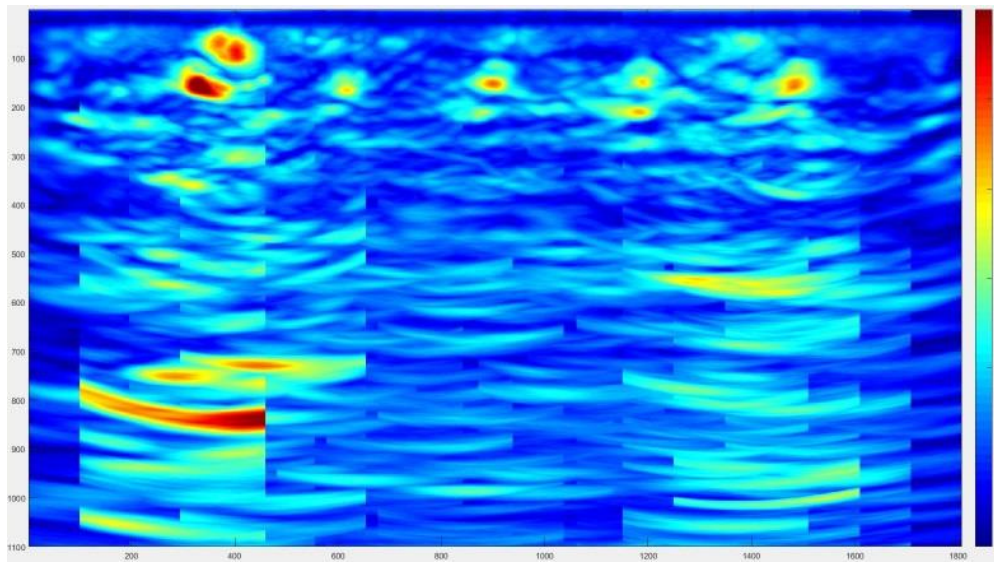

One half-period duration of the emitted signal

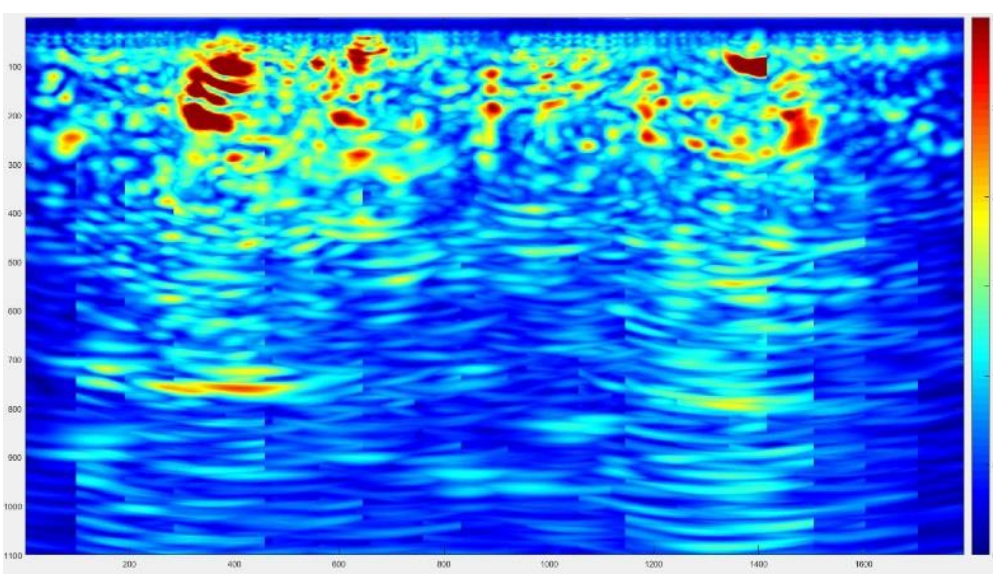

Four half-periods duration of the emitted signal

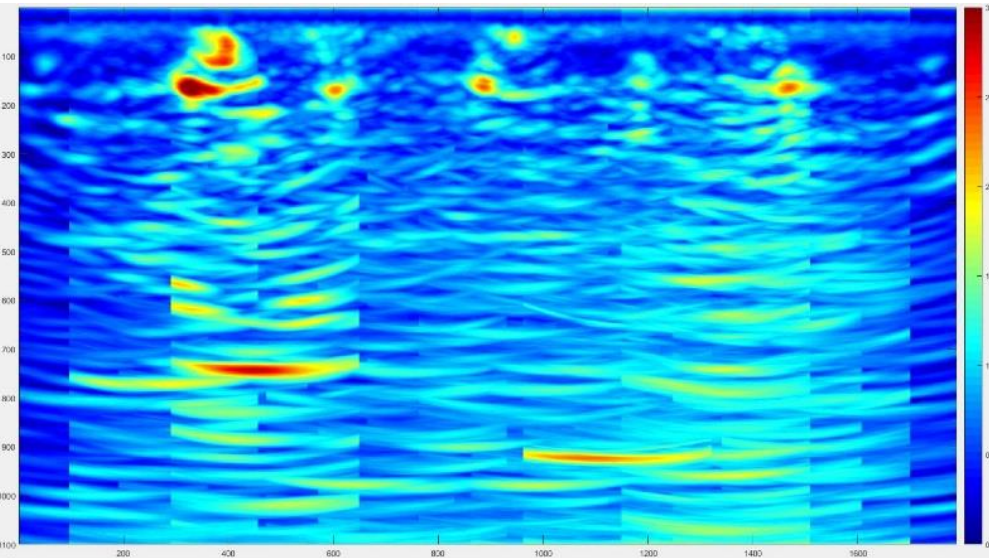

Two half-periods duration of the emitted signal

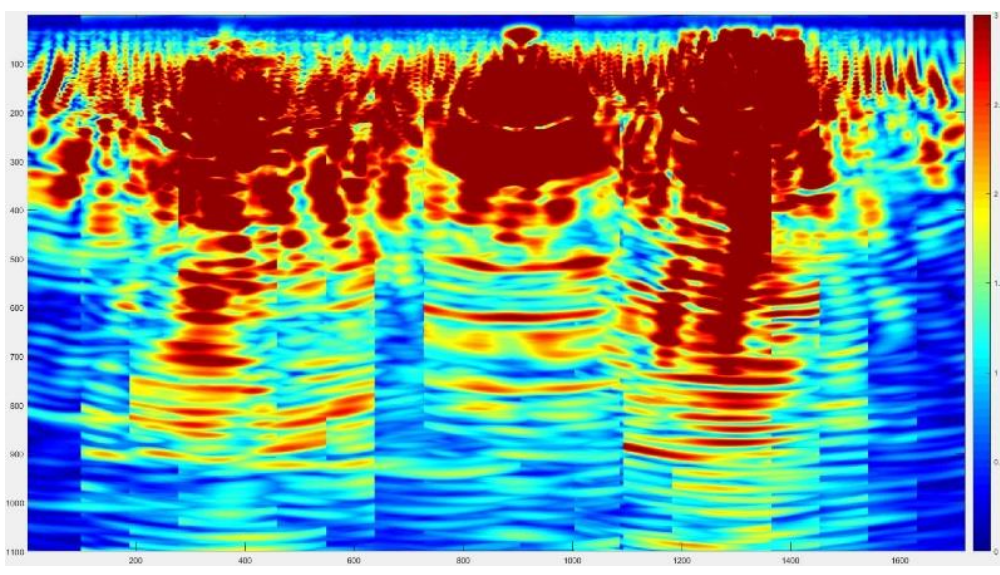

Sixteen half-periods duration of the emitted signal

Panoramic B-scan, row 14. 


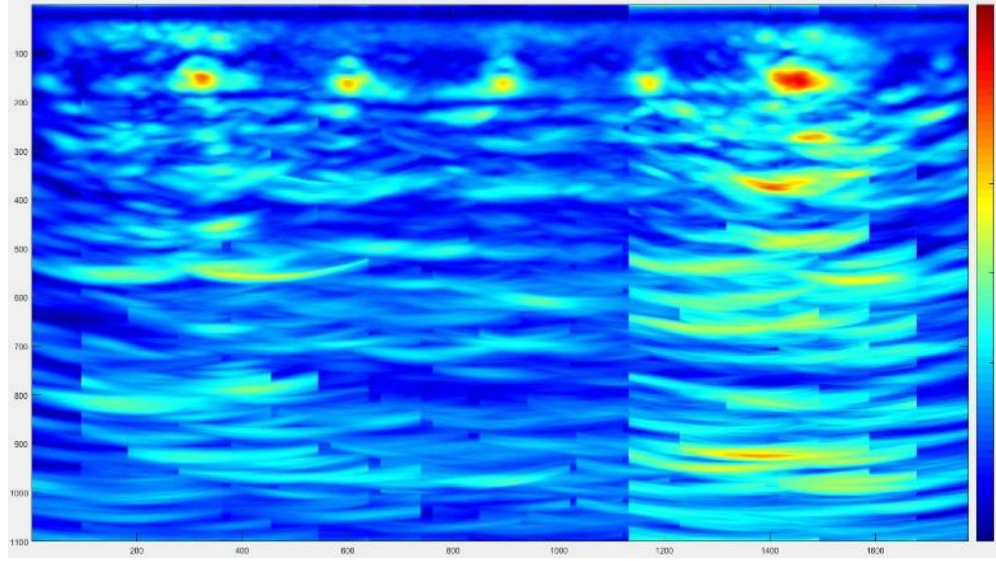

One half-period duration of the emitted signal

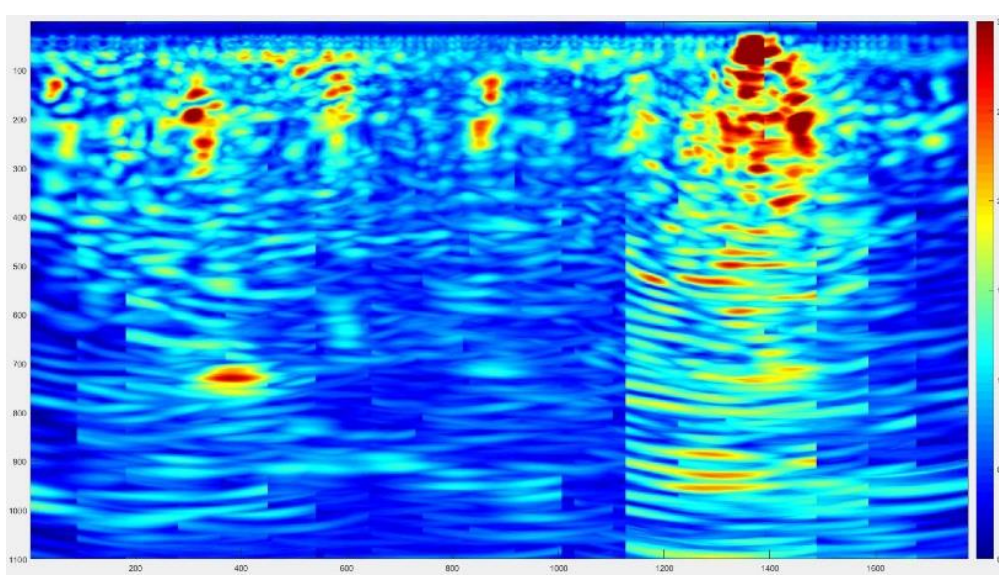

Four half-periods duration of the emitted signal

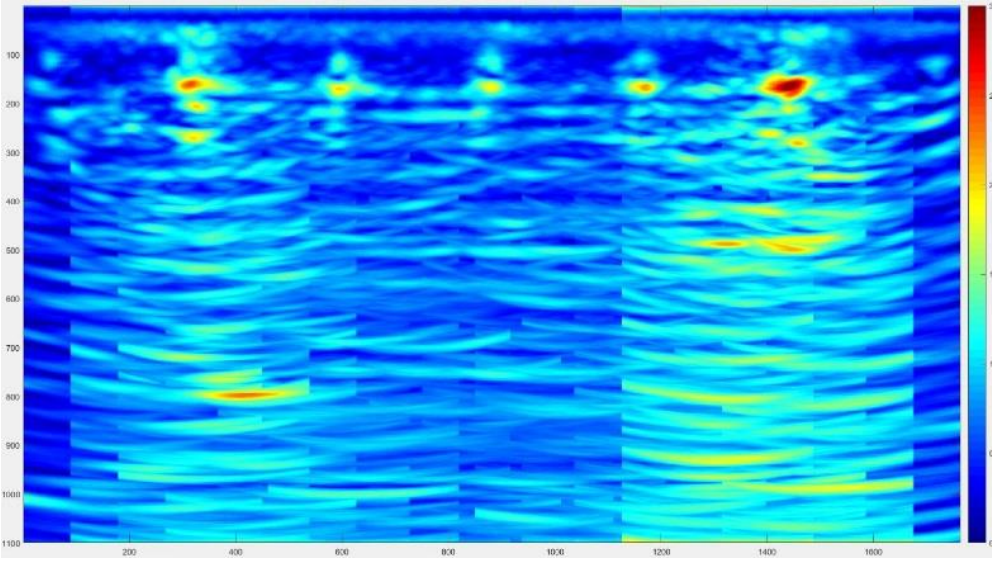

Two half-periods duration of the emitted signal

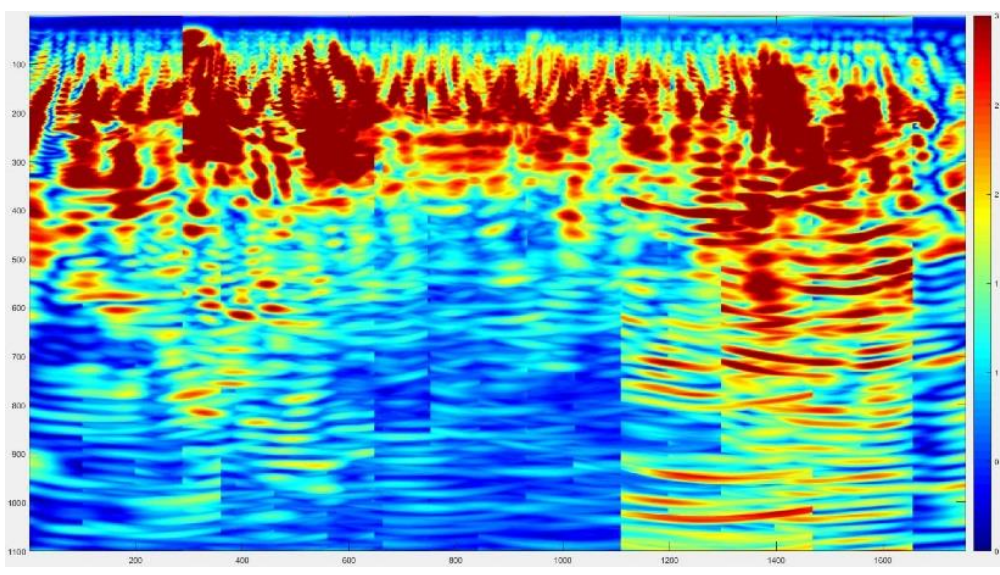

Sixteen half-periods duration of the emitted signal

Panoramic B-scan, row 15. 


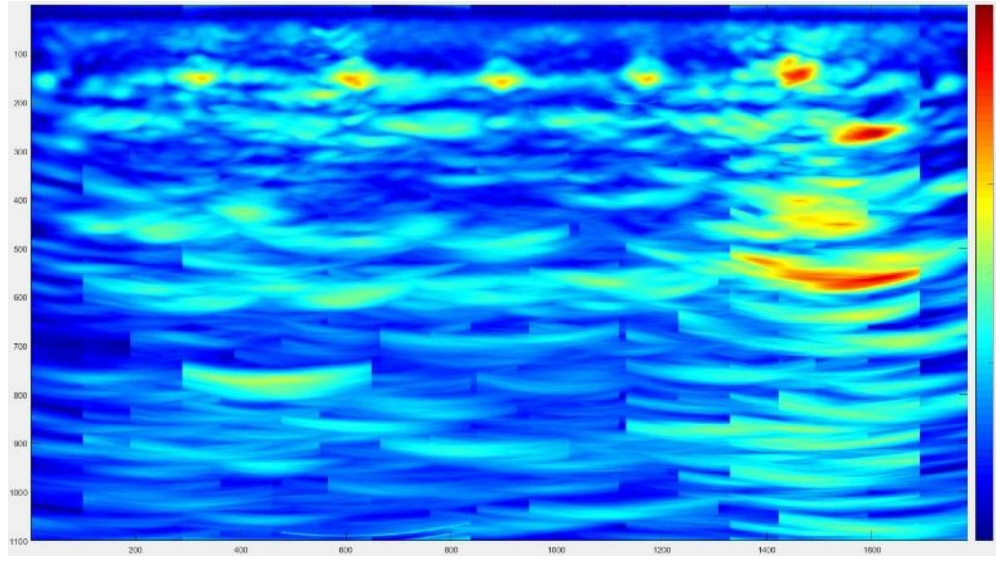

One half-period duration of the emitted signal

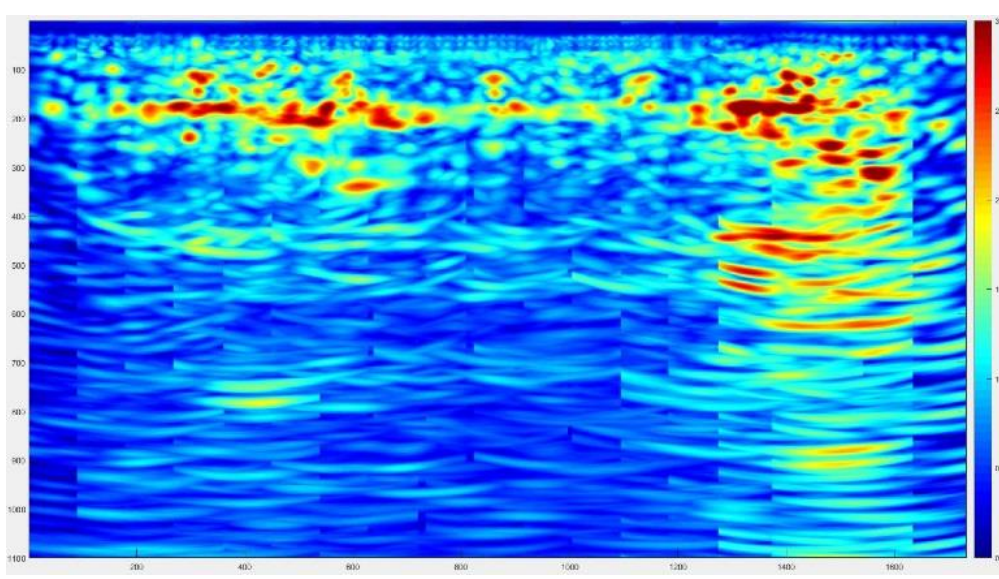

Four half-periods duration of the emitted signal

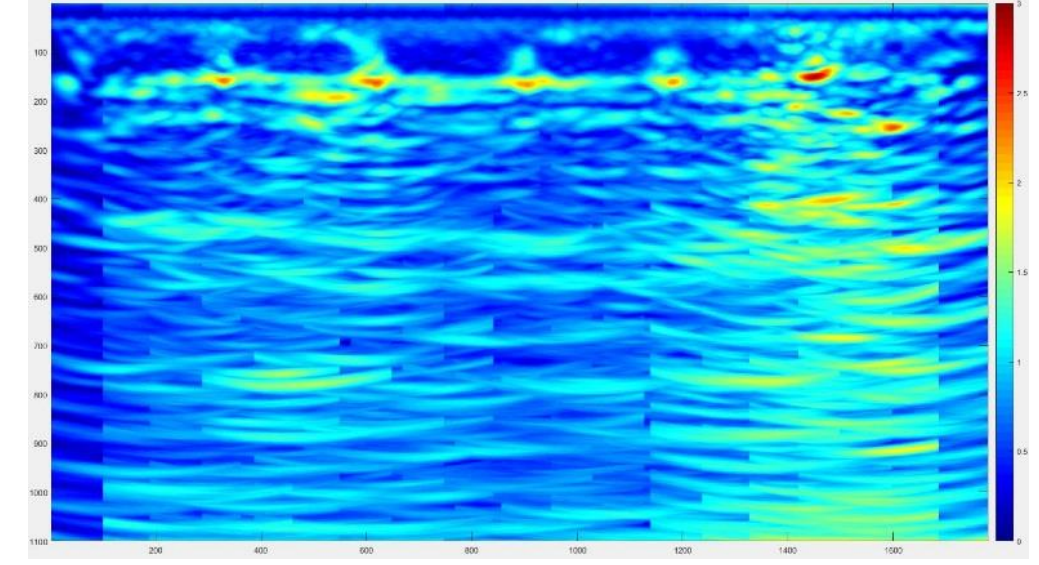

Two half-periods duration of the emitted signal

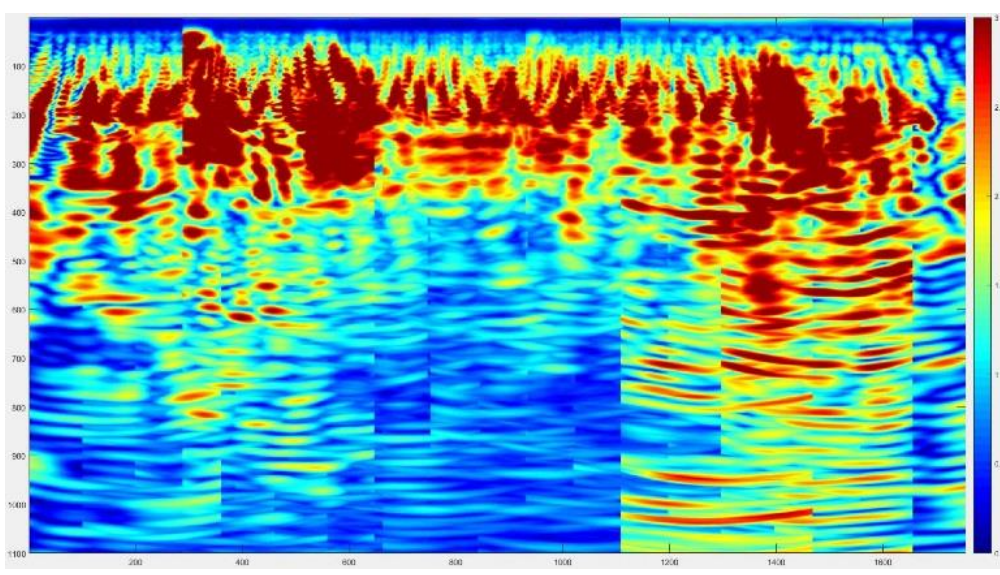

Sixteen half-periods duration of the emitted signal

Panoramic B-scan, row 16. 


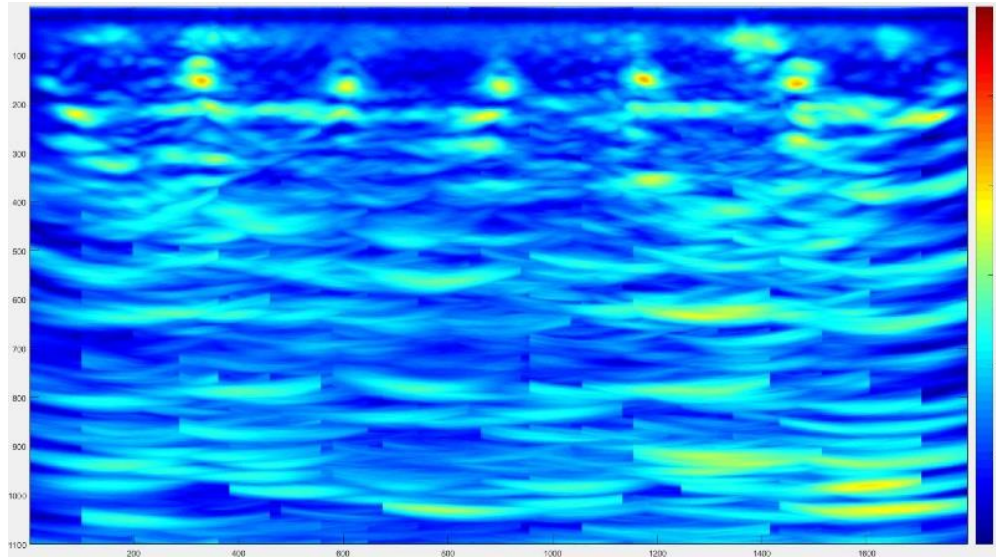

One half-period duration of the emitted signal

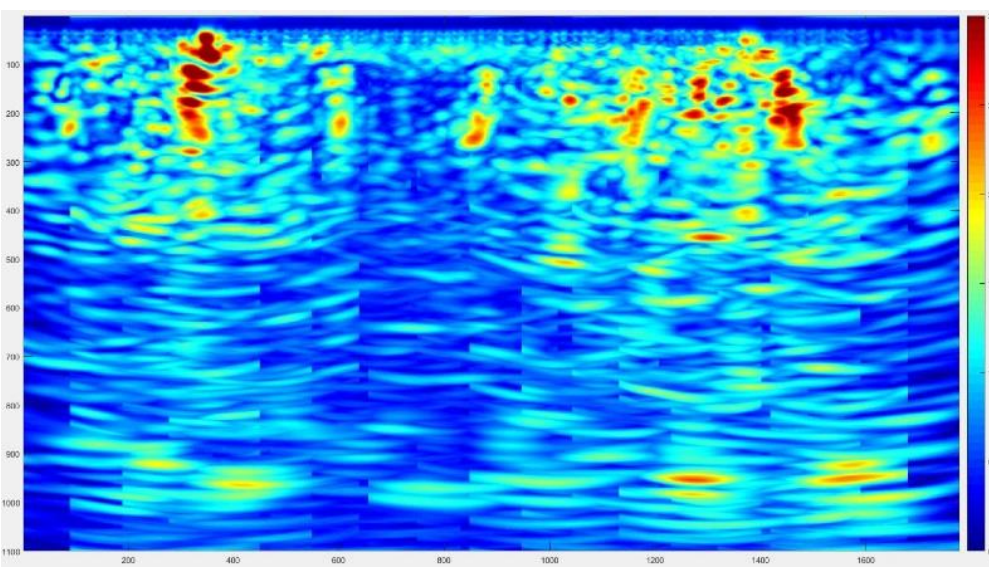

Four half-periods duration of the emitted signal

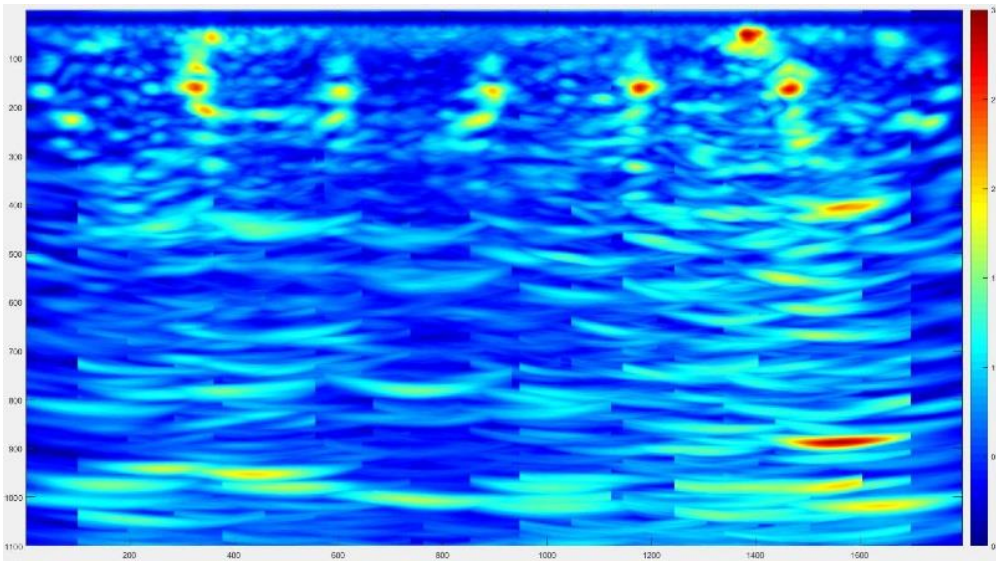

Two half-periods duration of the emitted signal

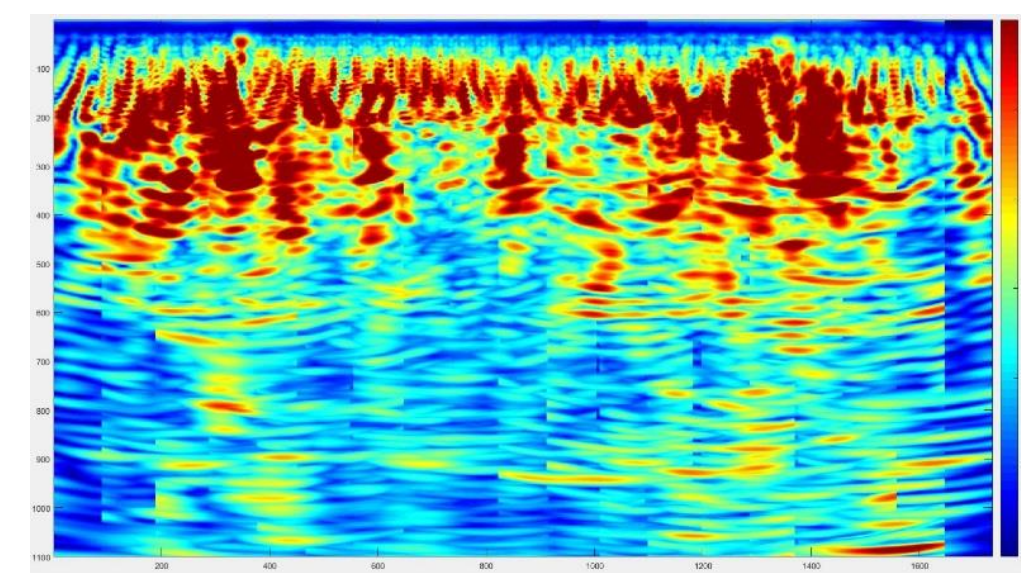

Sixteen half-periods duration of the emitted signal

Panoramic B-scan, row 17. 


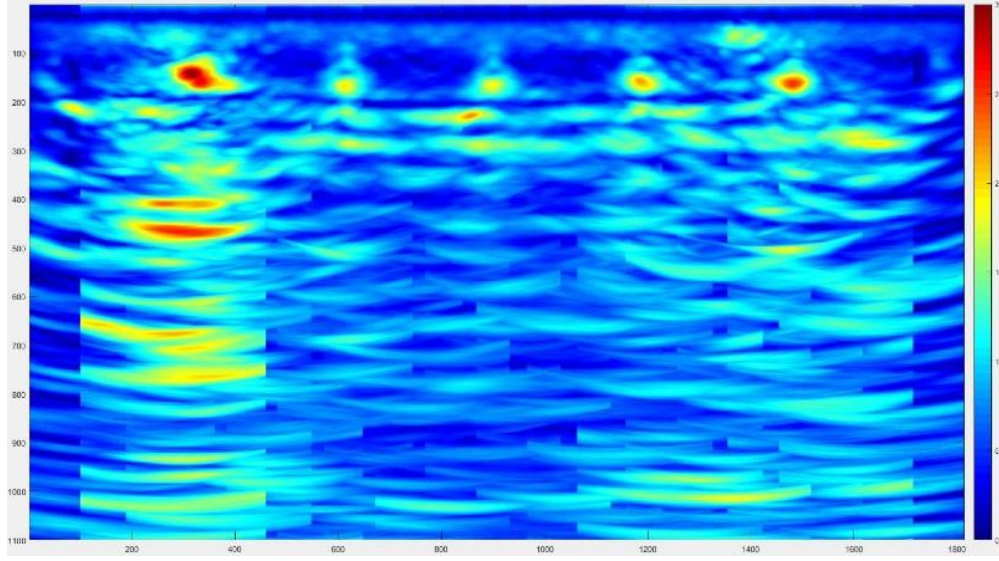

One half-period duration of the emitted signal

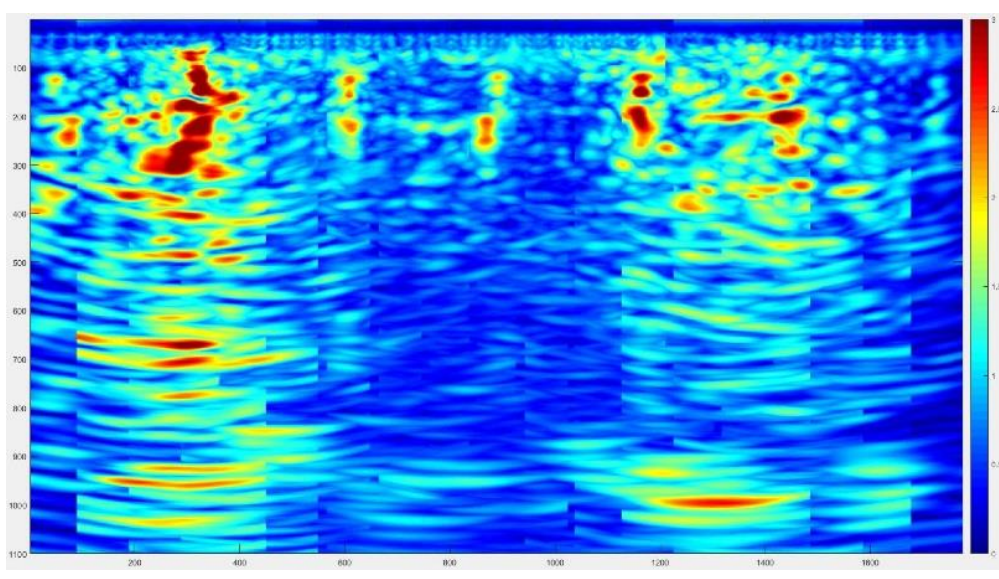

Four half-periods duration of the emitted signal

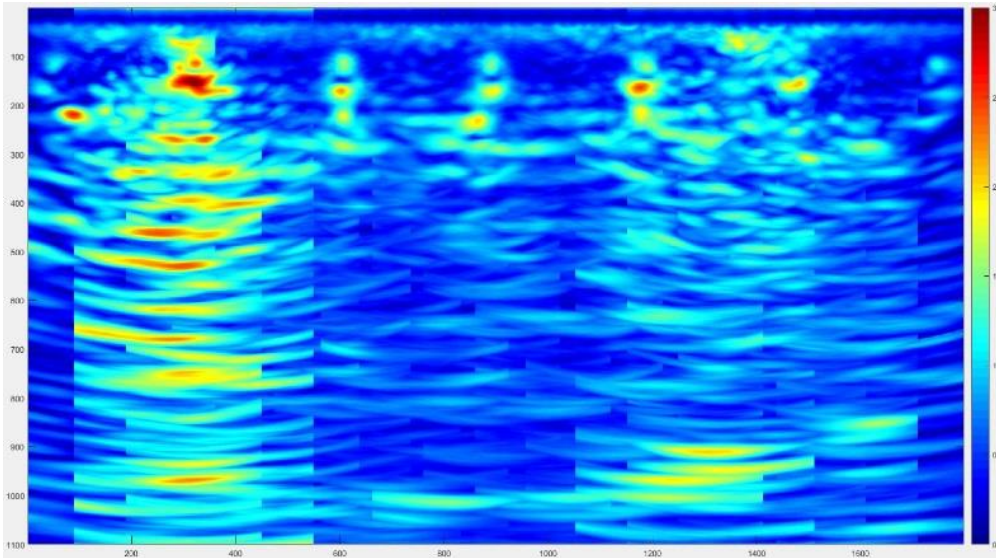

Two half-periods duration of the emitted signal

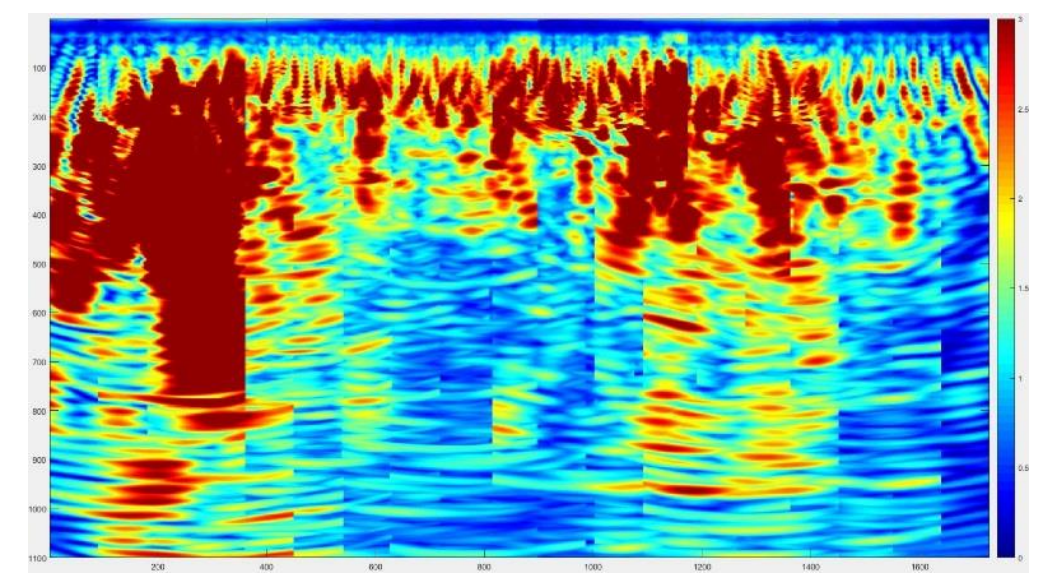

Sixteen half-periods duration of the emitted signal

Panoramic B-scan, row 18. 


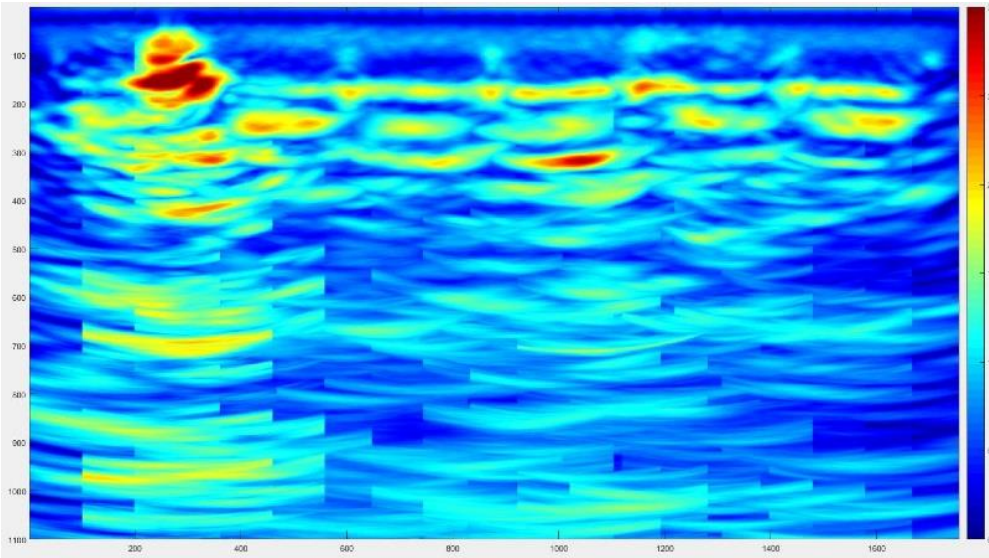

One half-period duration of the emitted signal

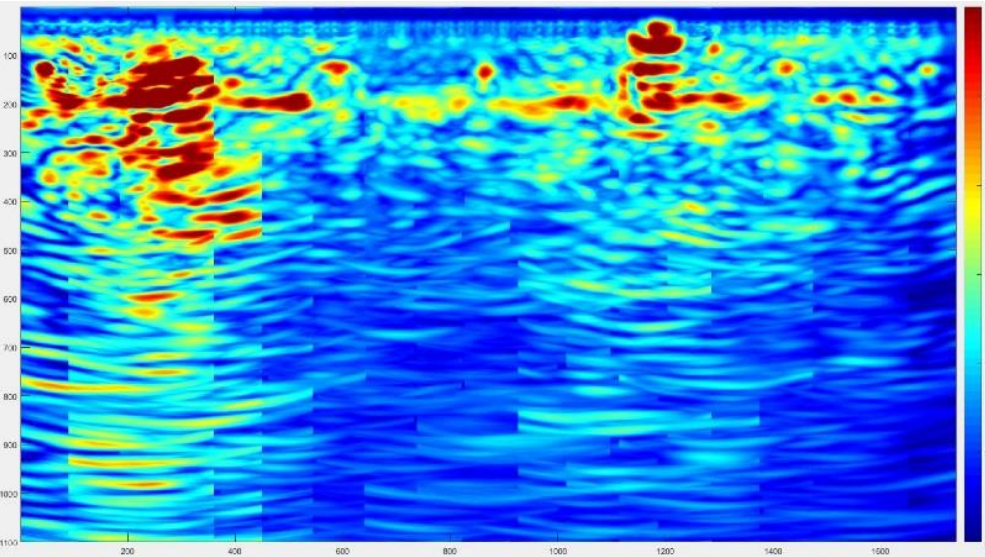

Four half-periods duration of the emitted signal

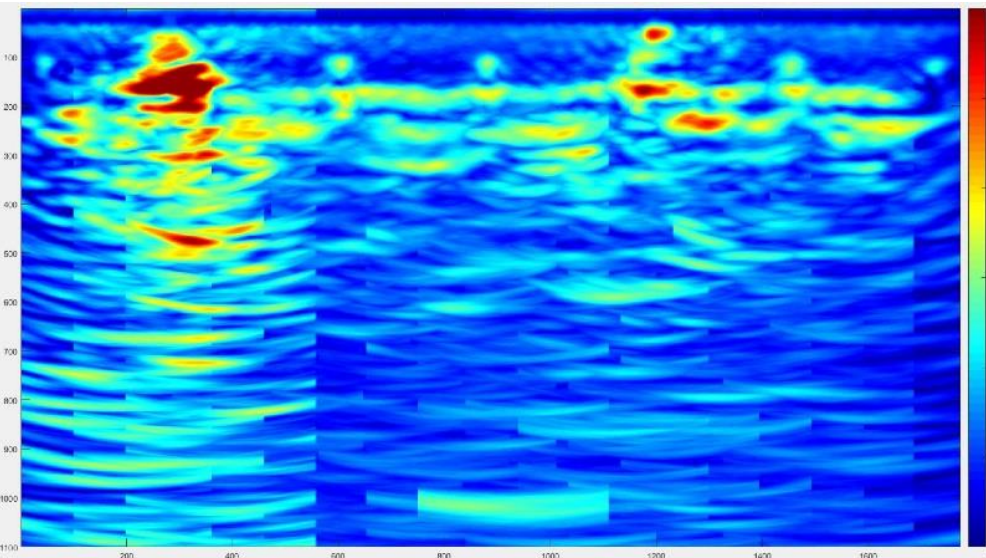

Two half-periods duration of the emitted signal

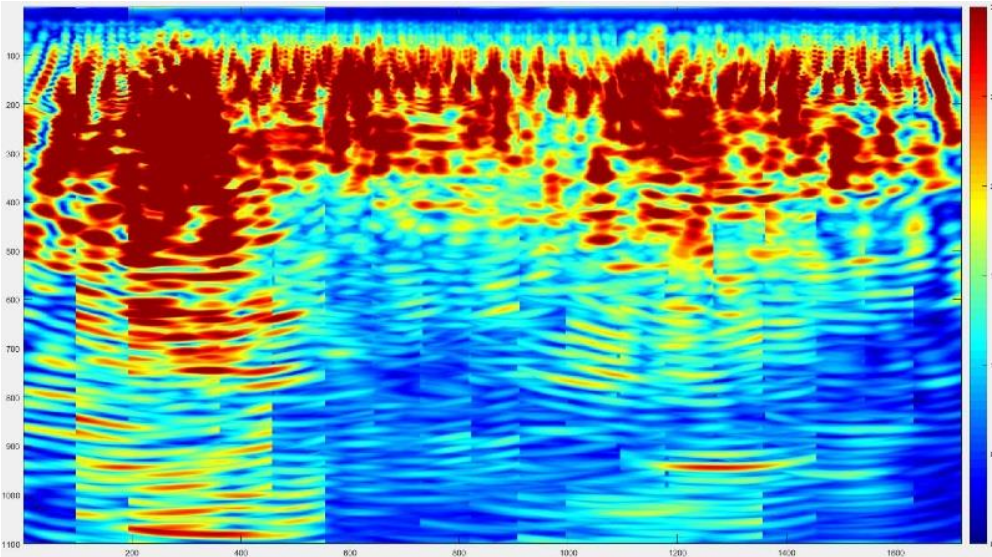

Sixteen half-periods duration of the emitted signal

Panoramic B-scan, row 19. 





\section{APPENDIX C. PANORAMIC IMAGES FOR LENGTH ORIENTATION}

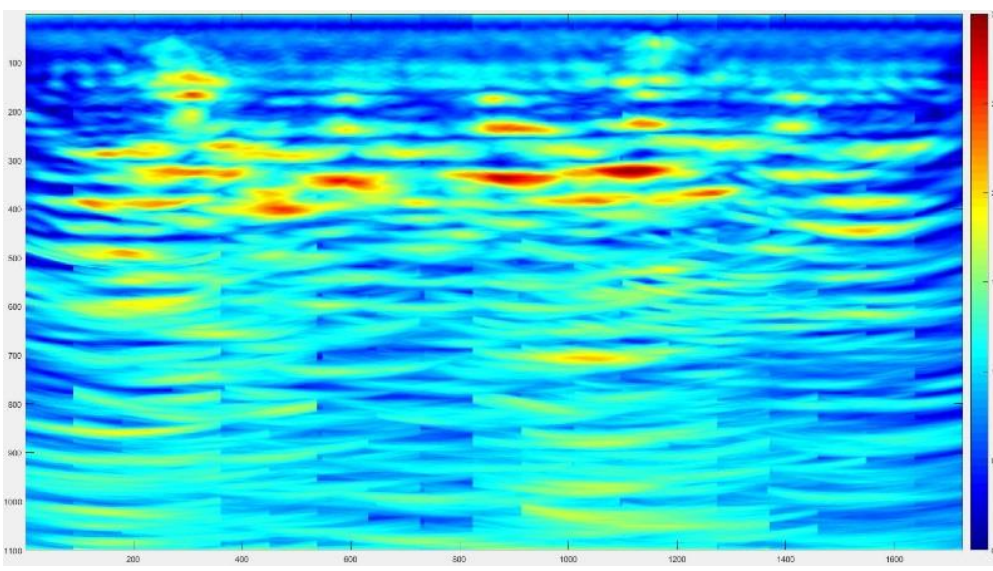

One half-period duration of the emitted signal

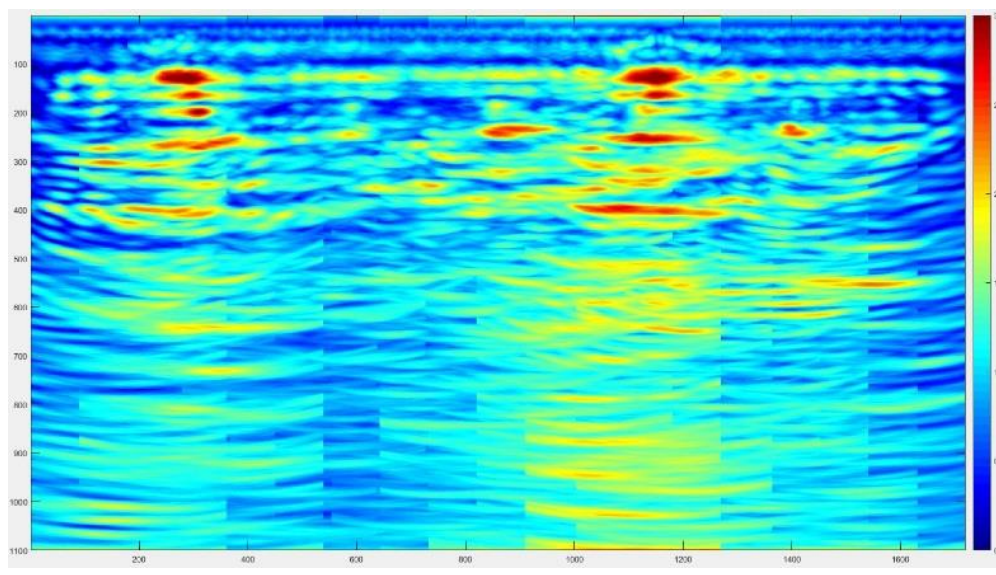

Four half-periods duration of the emitted signal

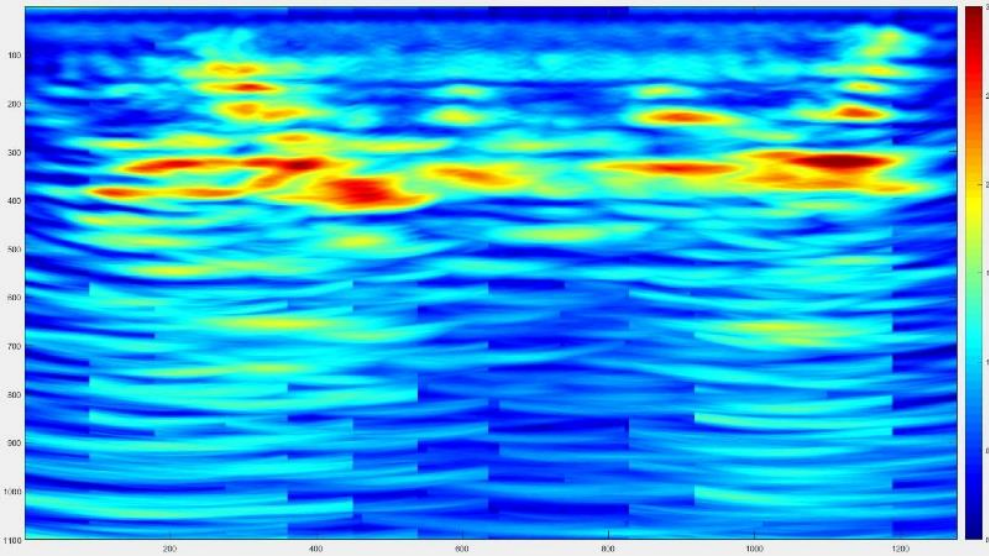

Two half-periods duration of the emitted signal

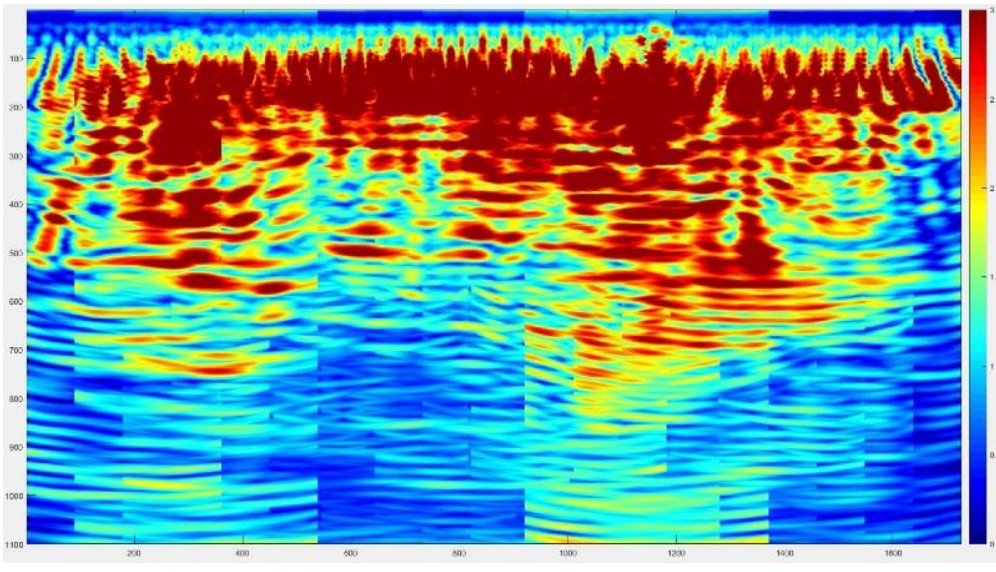

Sixteen half-periods duration of the emitted signal

Panoramic B-scan, row 1. 


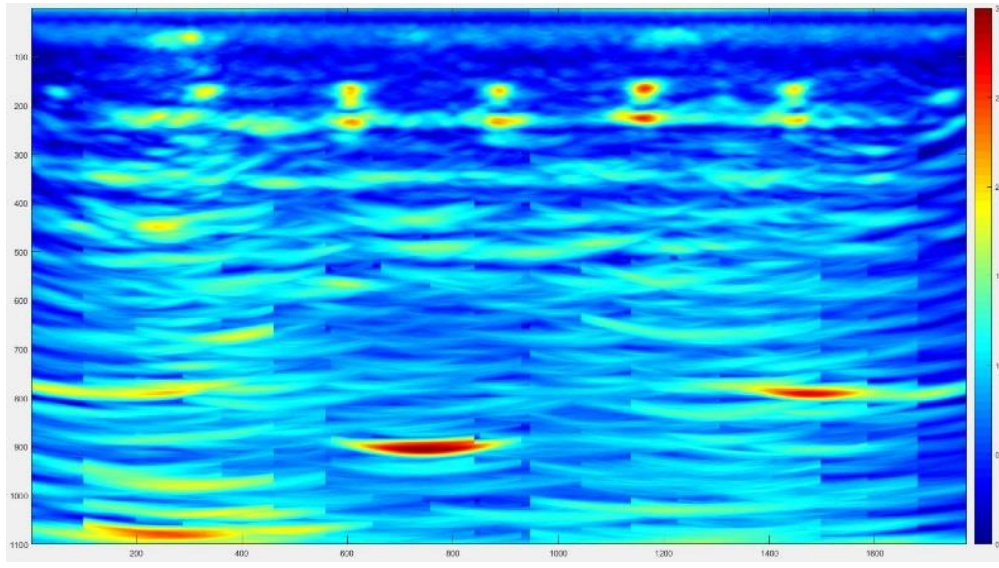

One half-period duration of the emitted signal

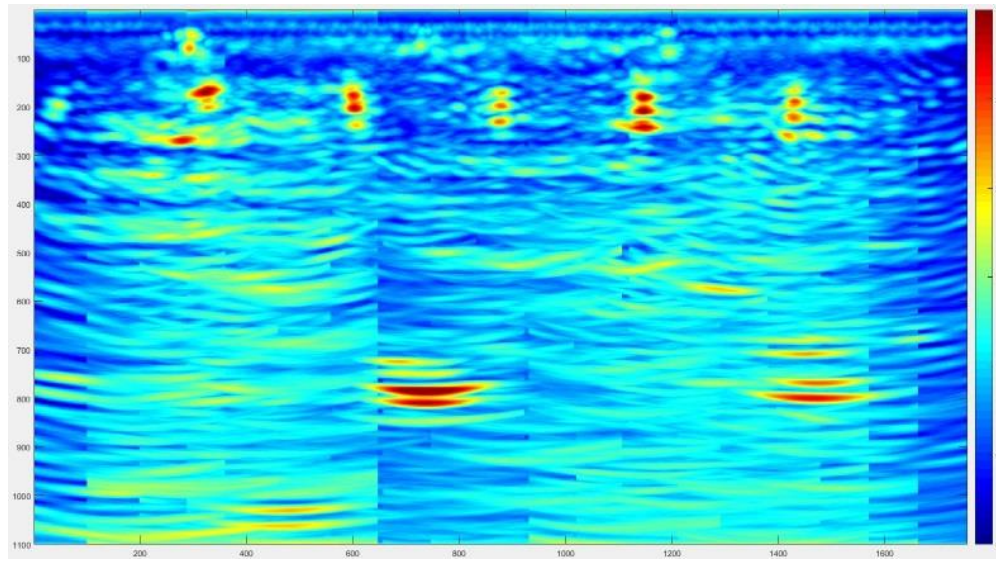

Four half-periods duration of the emitted signal

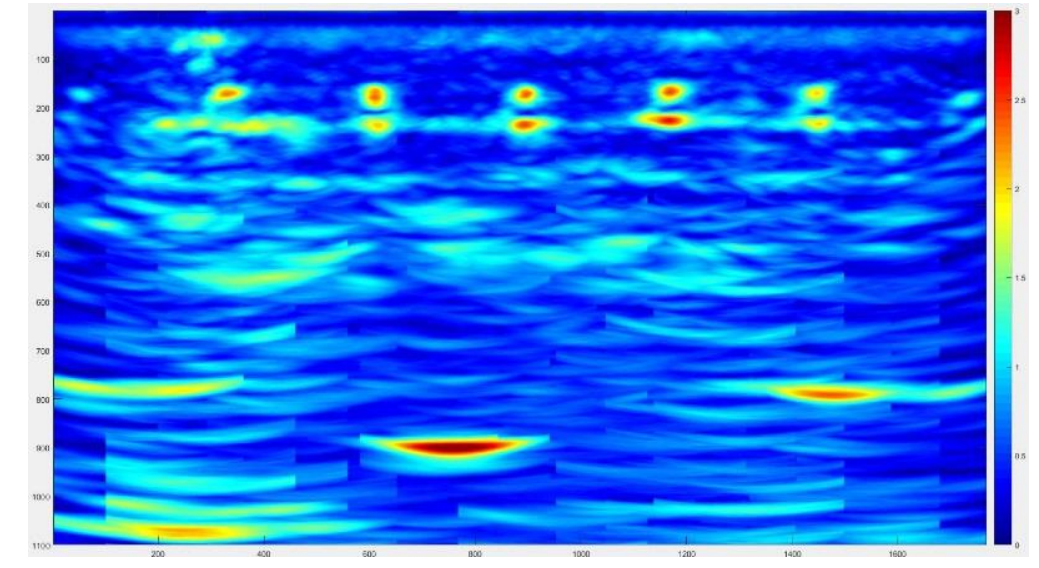

Two half-periods duration of the emitted signal

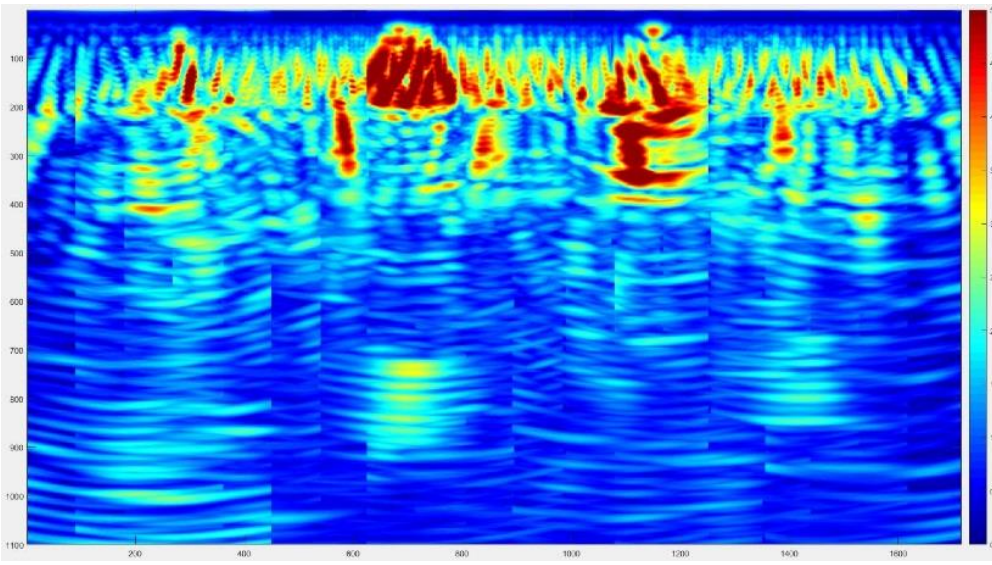

Sixteen half-periods duration of the emitted signal

Panoramic B-scan, row 2. 


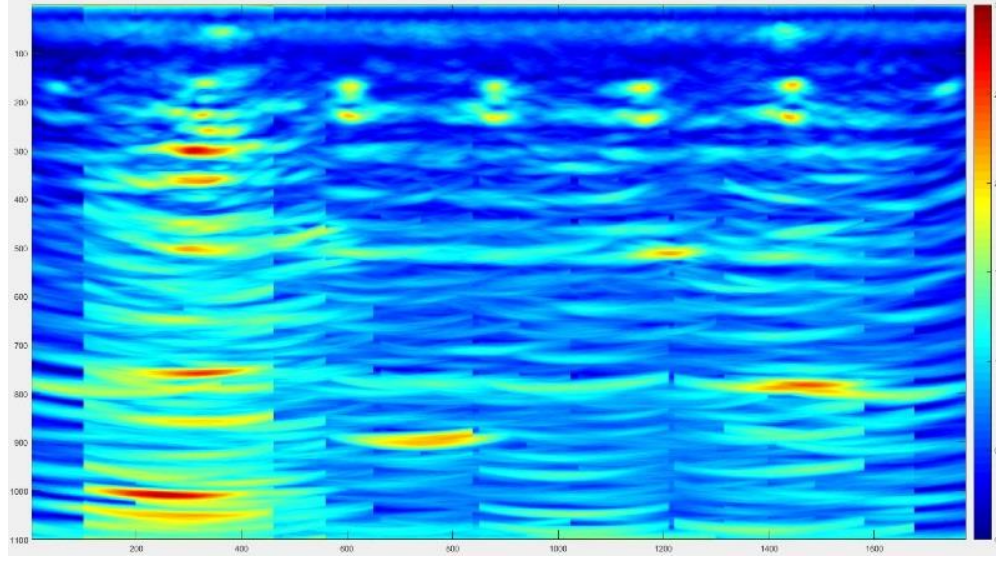

One half-period duration of the emitted signal

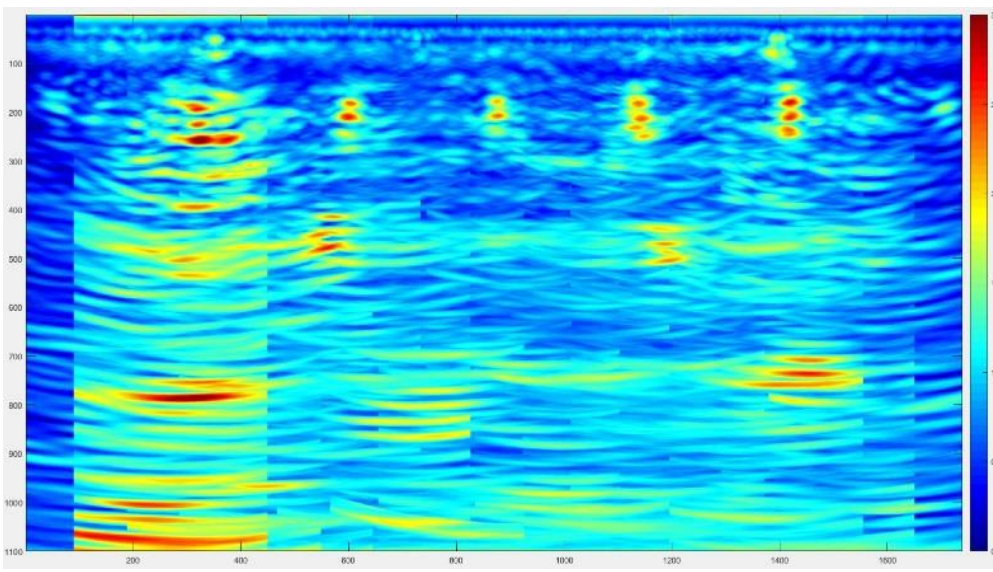

Four half-periods duration of the emitted signal

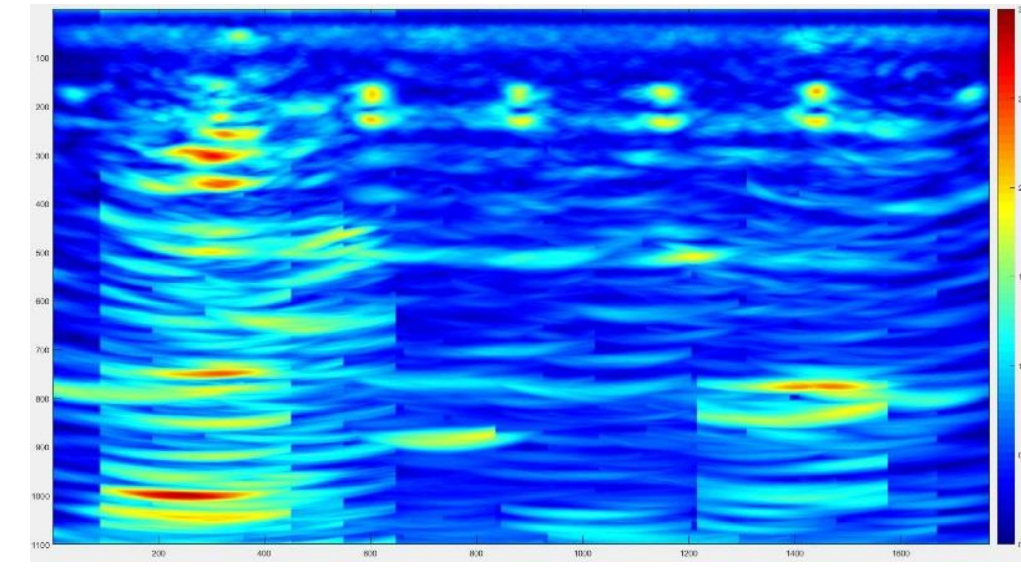

Two half-periods duration of the emitted signal

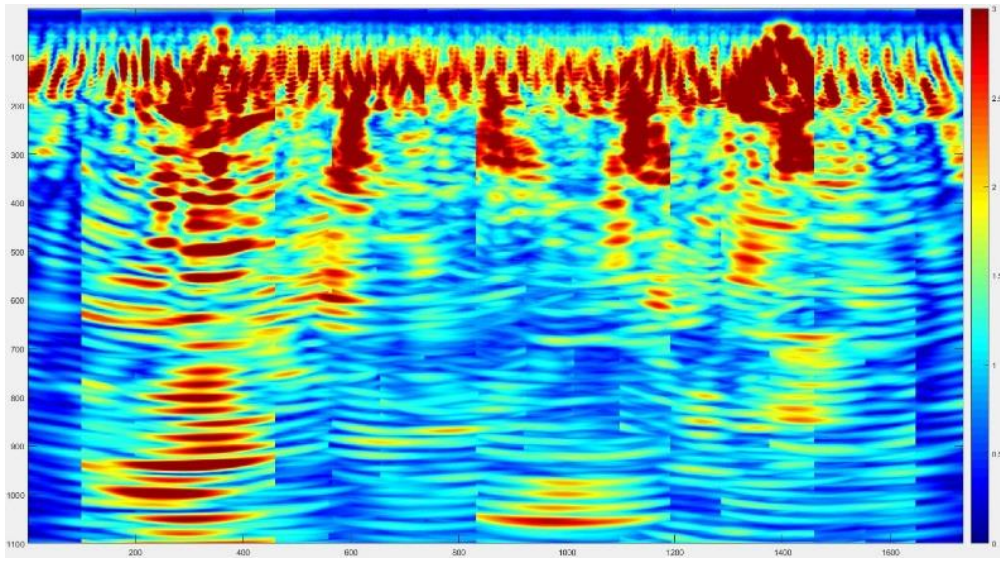

Sixteen half-periods duration of the emitted signal

Panoramic B-scan, row 3. 


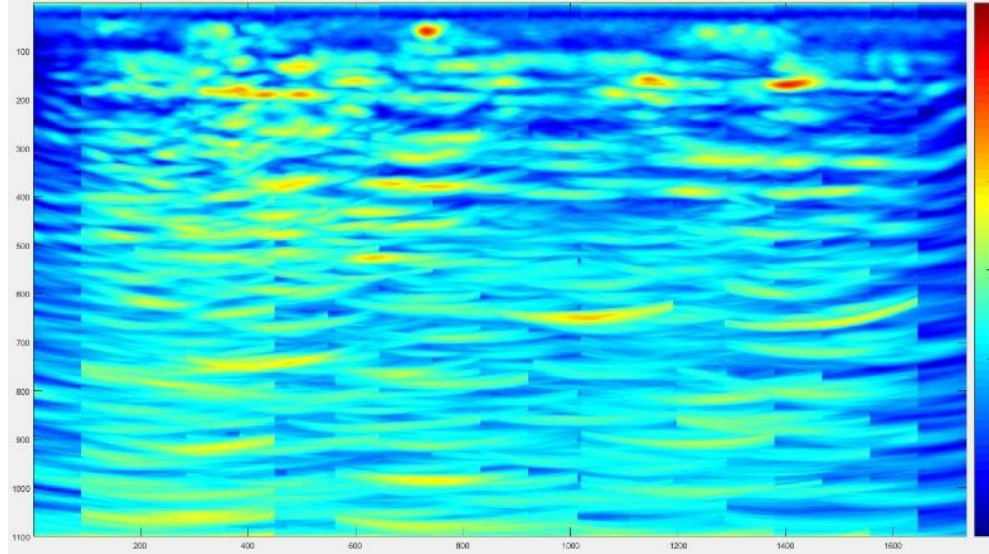

One half-period duration of the emitted signal

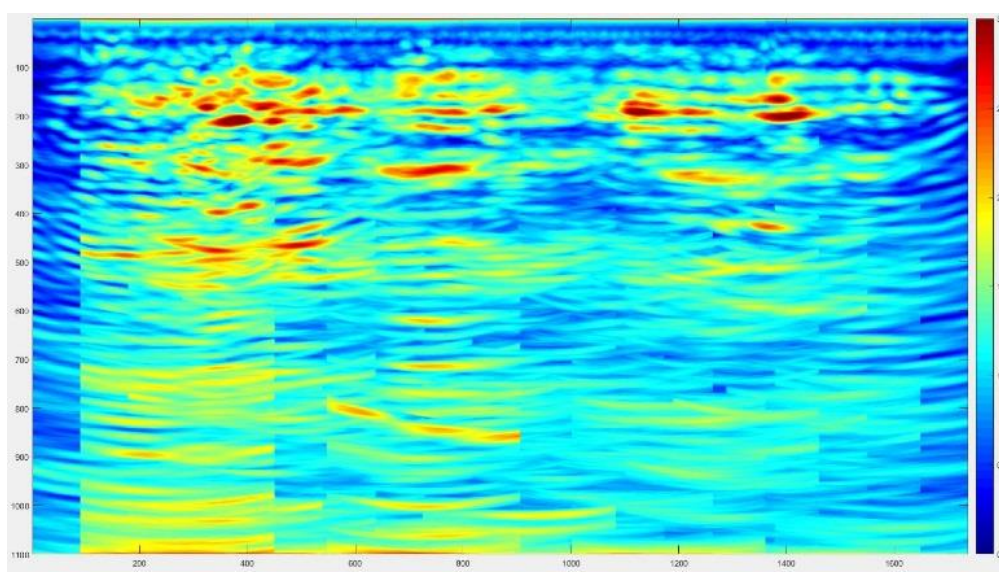

Four half-periods duration of the emitted signal

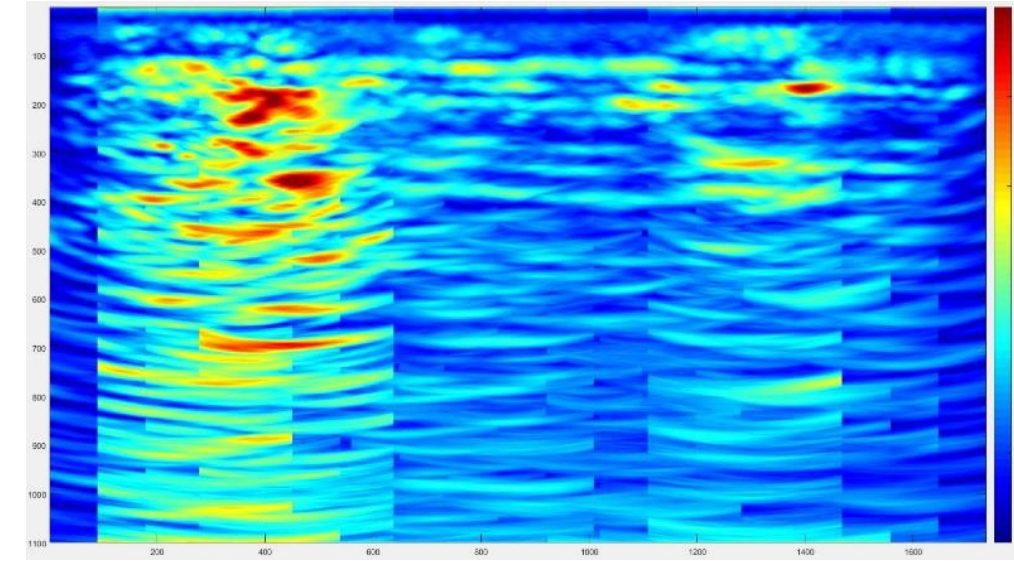

Two half-periods duration of the emitted signal

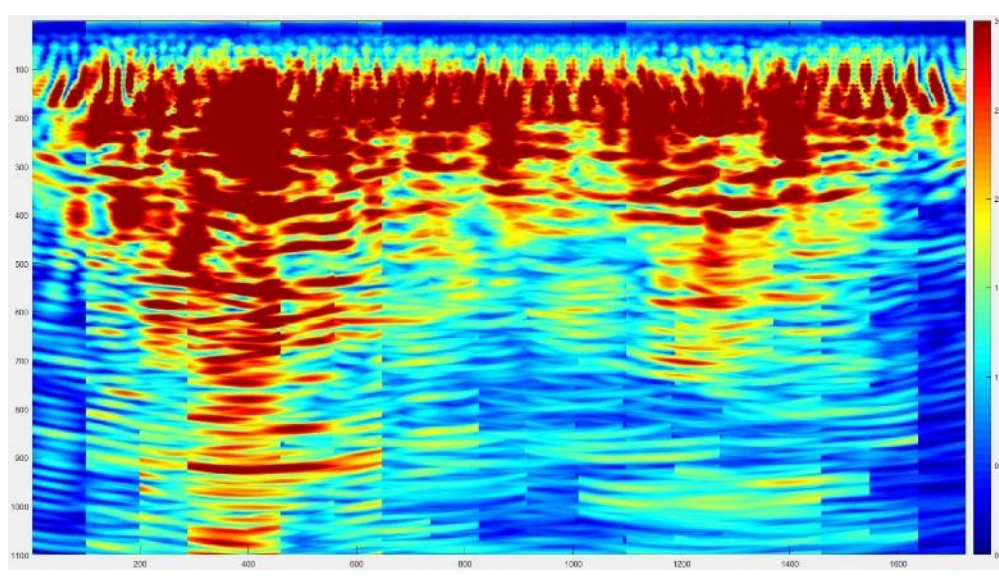

Sixteen half-periods duration of the emitted signal

Panoramic B-scan, row 4. 


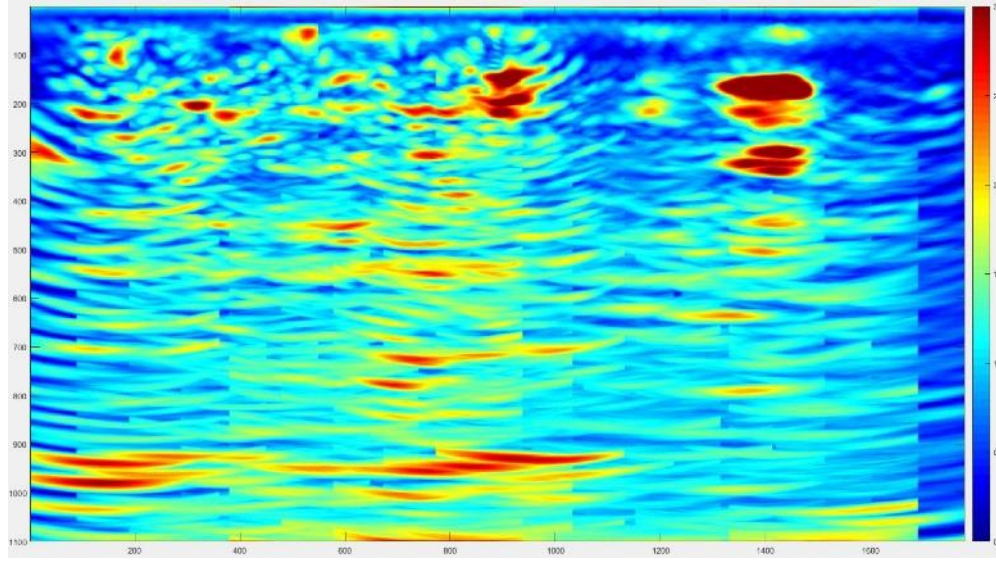

One half-period duration of the emitted signal

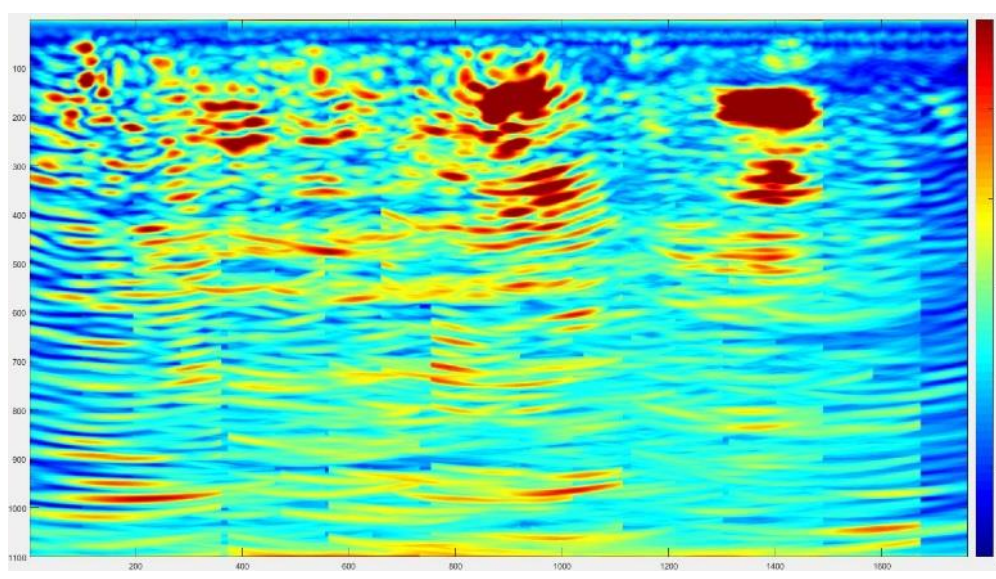

Four half-periods duration of the emitted signal

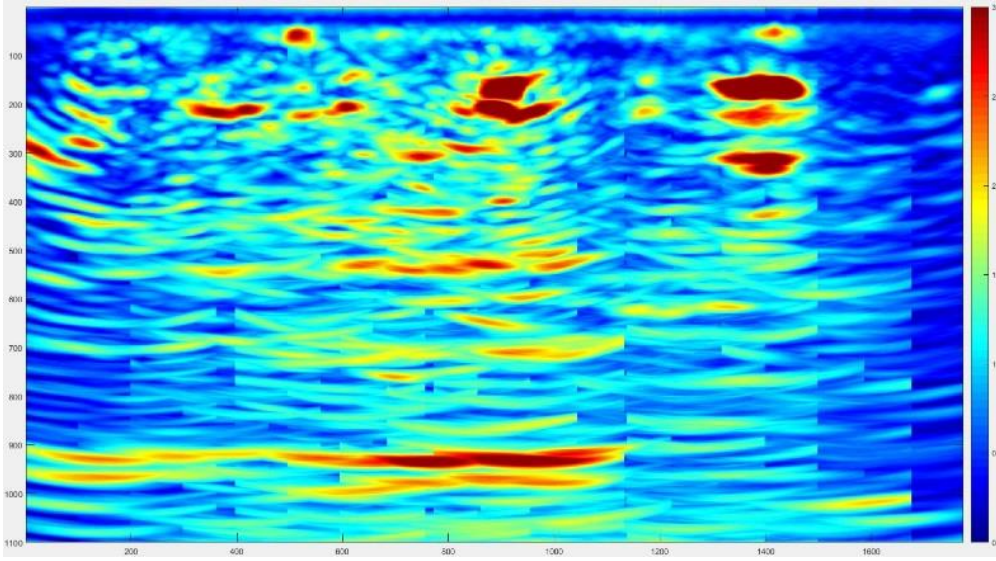

Two half-periods duration of the emitted signal

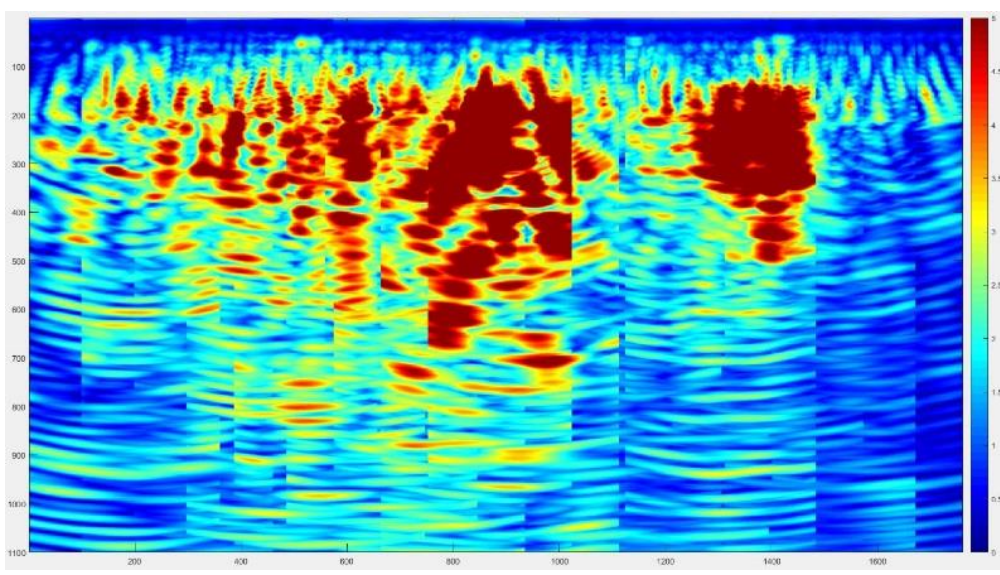

Sixteen half-periods duration of the emitted signal

Panoramic B-scan, row 5. 


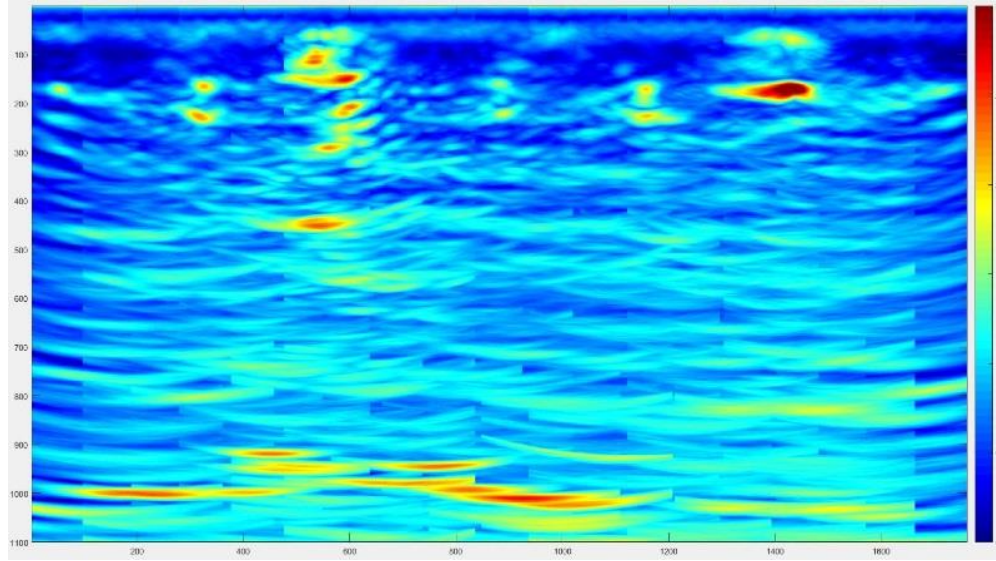

One half-period duration of the emitted signal

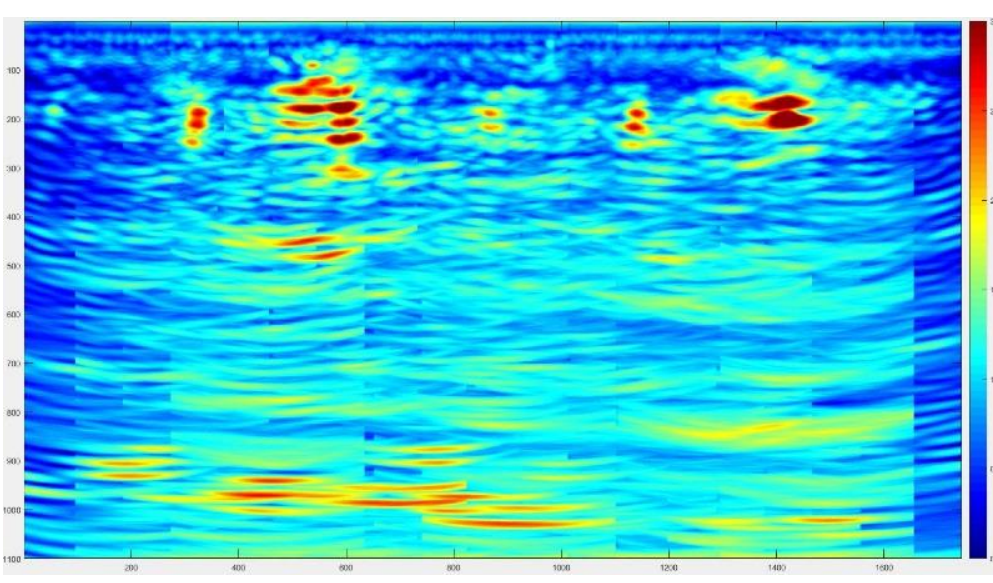

Four half-periods duration of the emitted signal

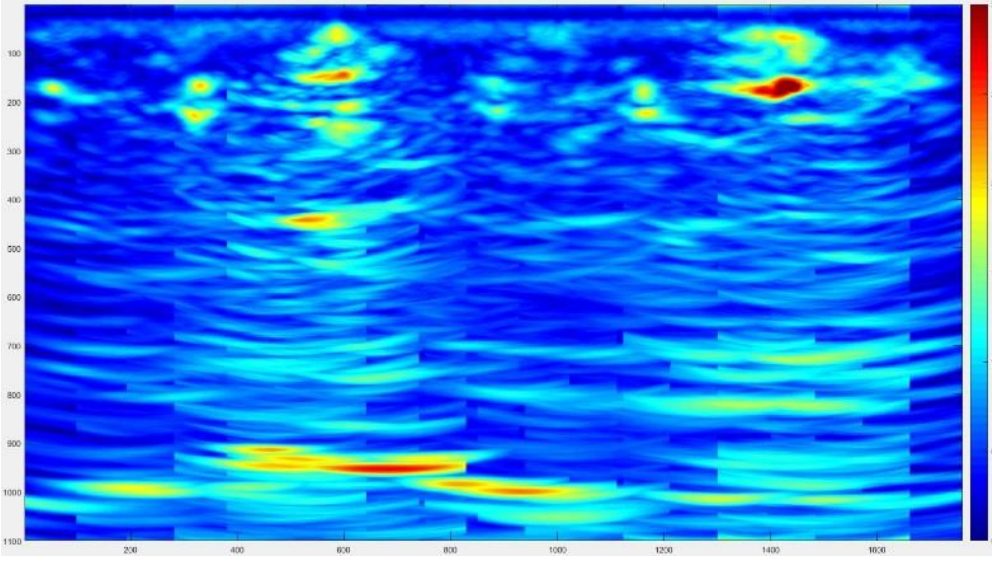

Two half-periods duration of the emitted signal

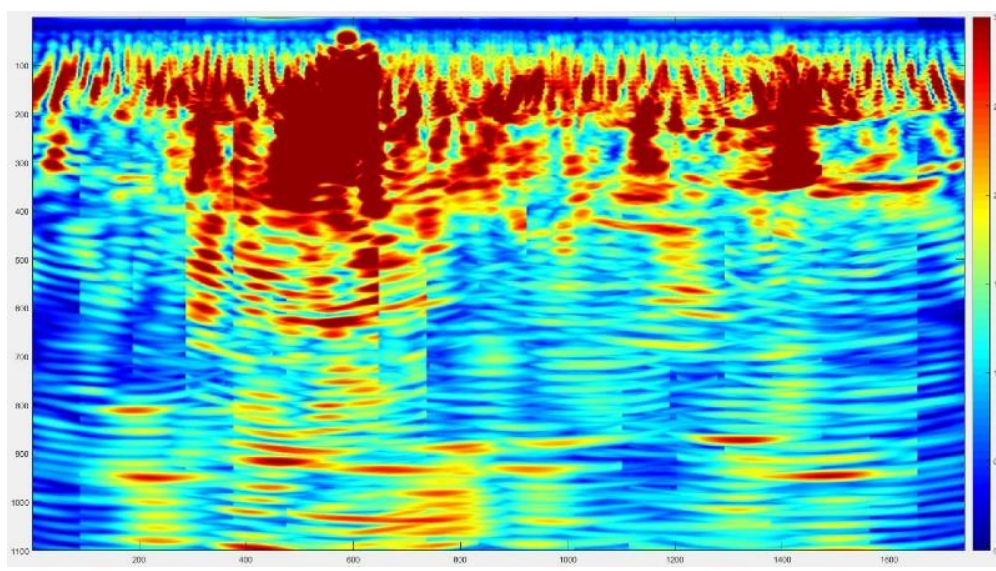

Sixteen half-periods duration of the emitted signal

Panoramic B-scan, row 6. 


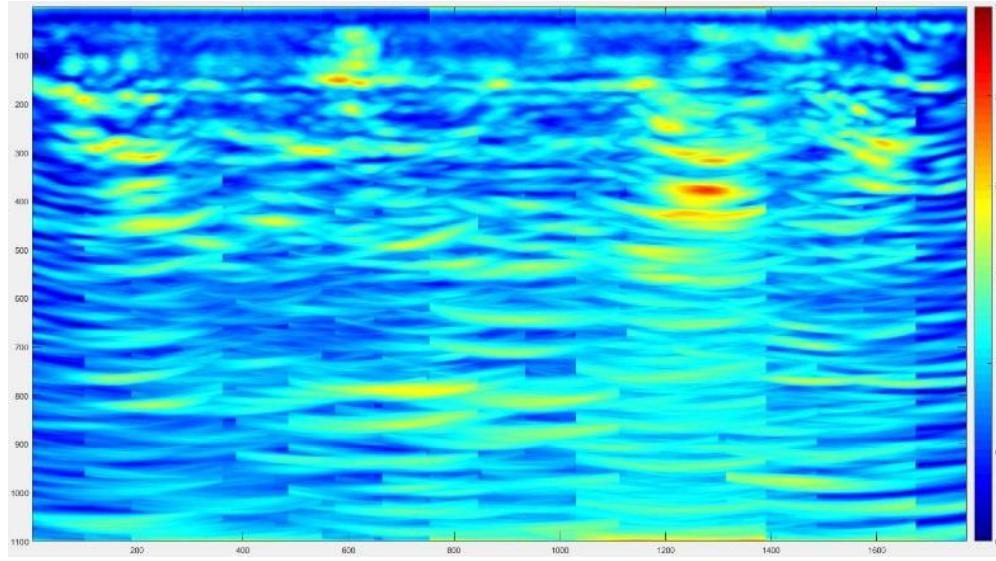

One half-period duration of the emitted signal

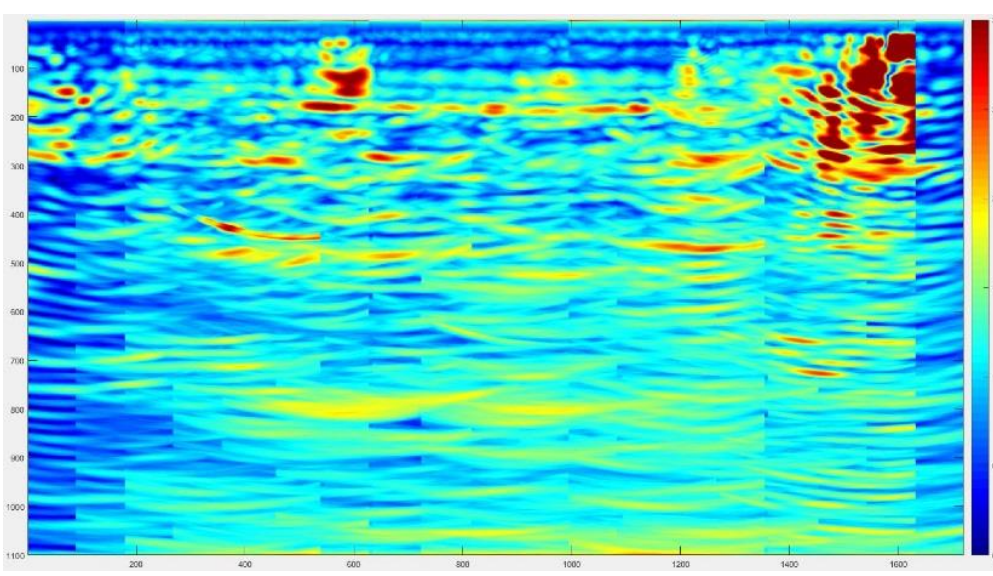

Four half-periods duration of the emitted signal

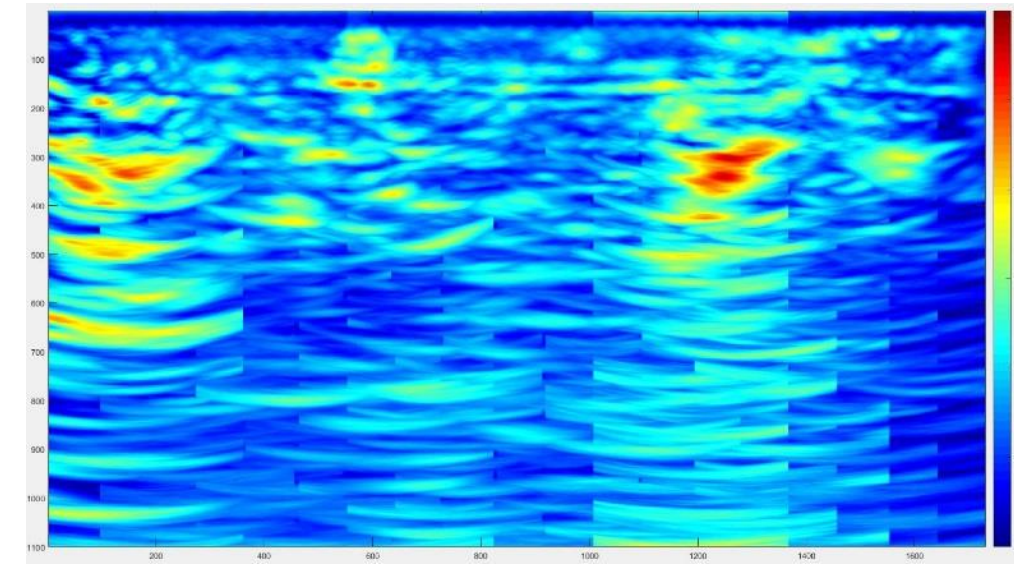

Two half-periods duration of the emitted signal

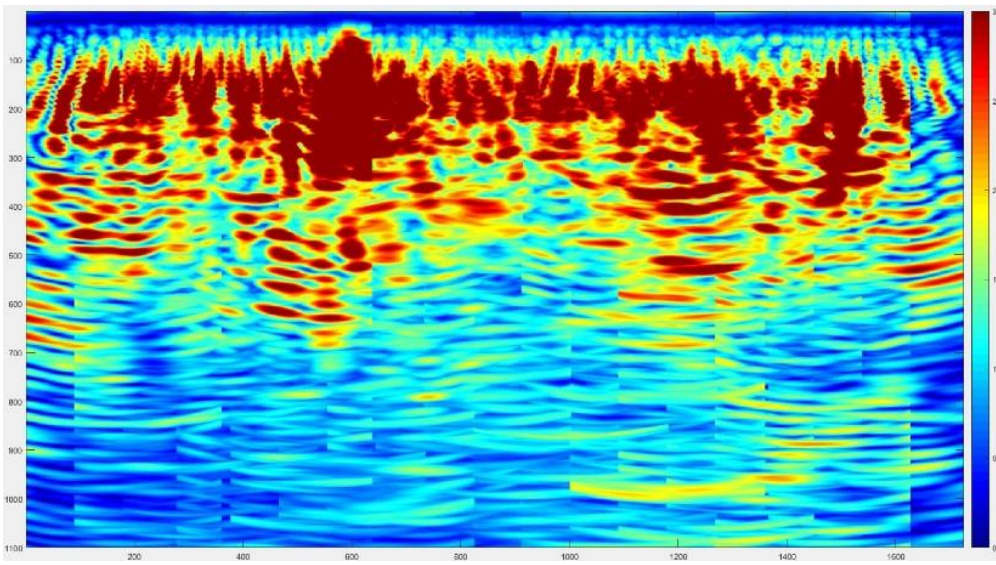

Sixteen half-periods duration of the emitted signal

Panoramic B-scan, row 7. 


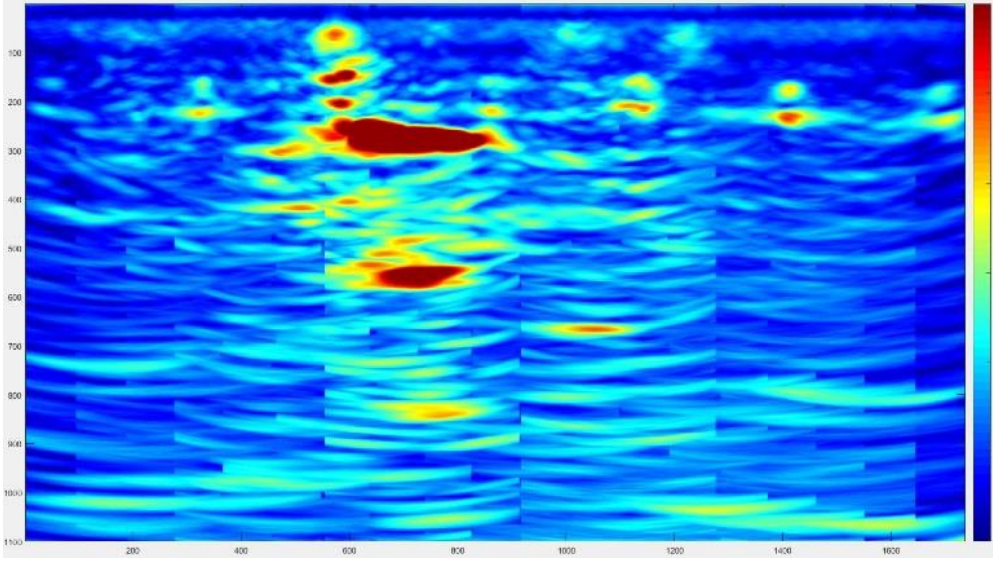

One half-period duration of the emitted signal

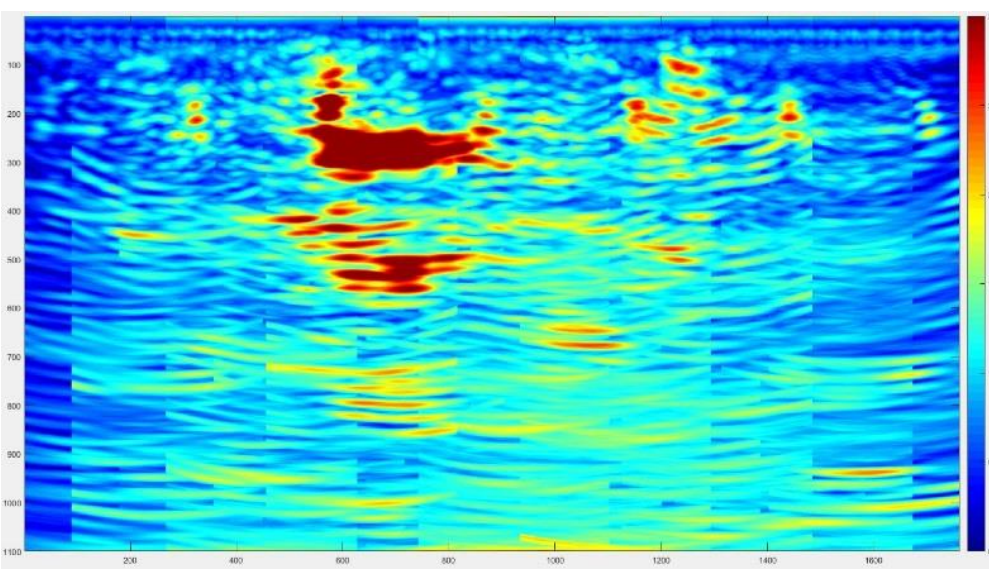

Four half-periods duration of the emitted signal

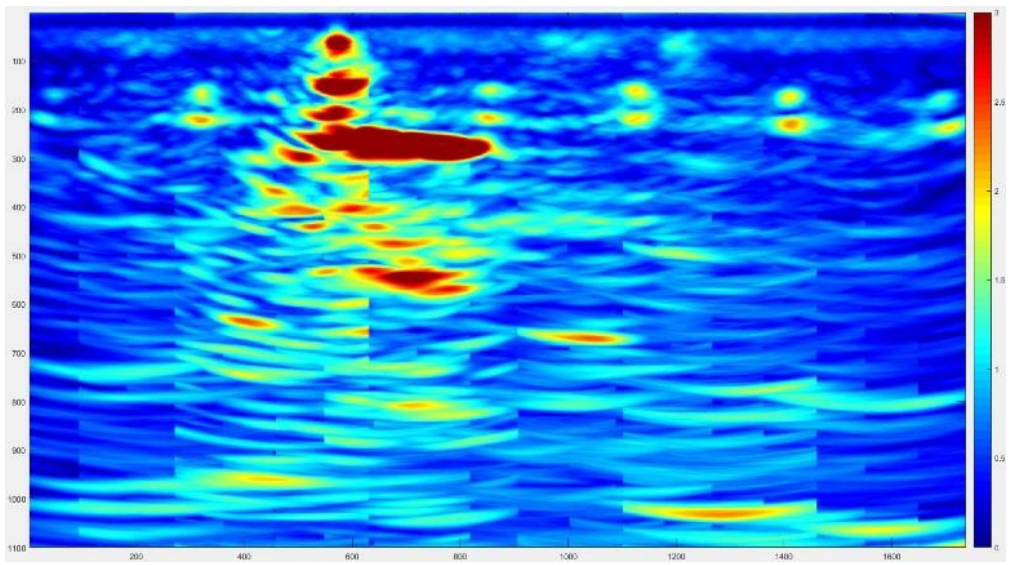

Two half-periods duration of the emitted signal

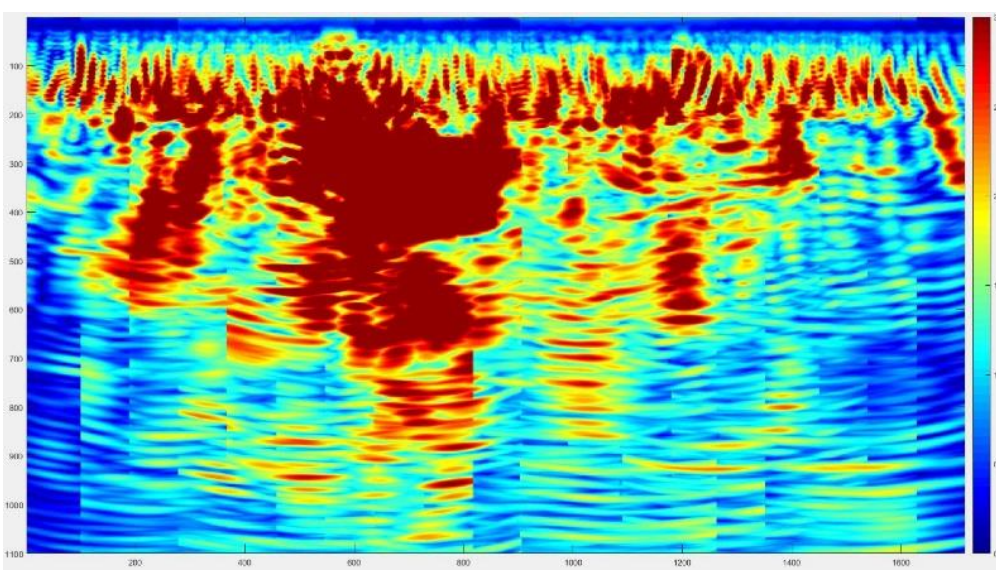

Sixteen half-periods duration of the emitted signal

Panoramic B-scan, row 8. 


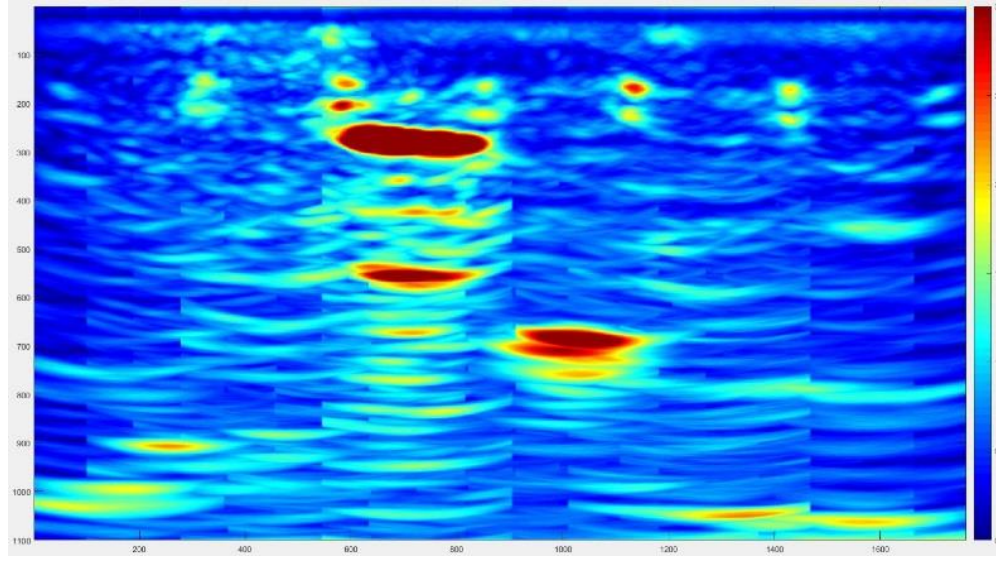

One half-period duration of the emitted signal

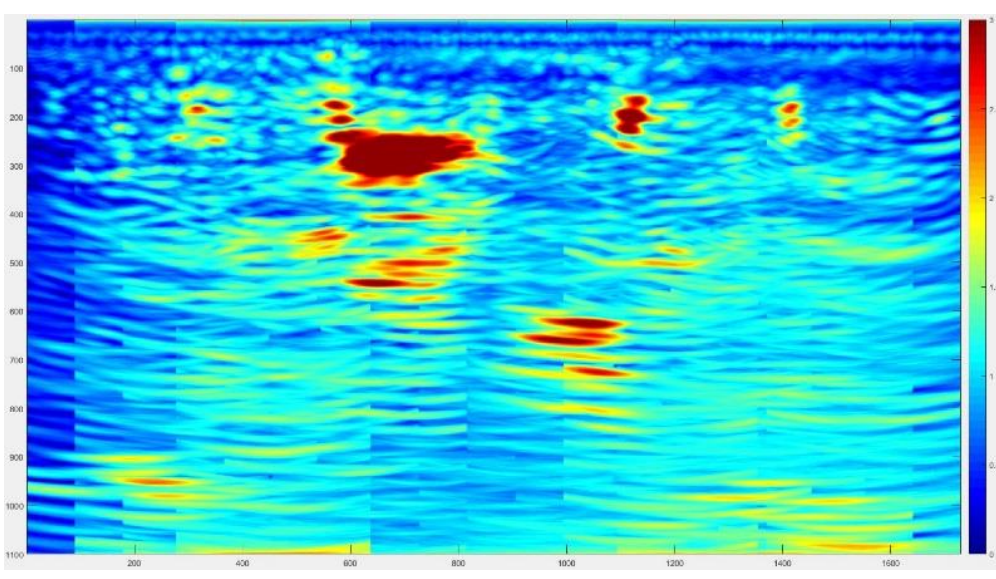

Four half-periods duration of the emitted signal

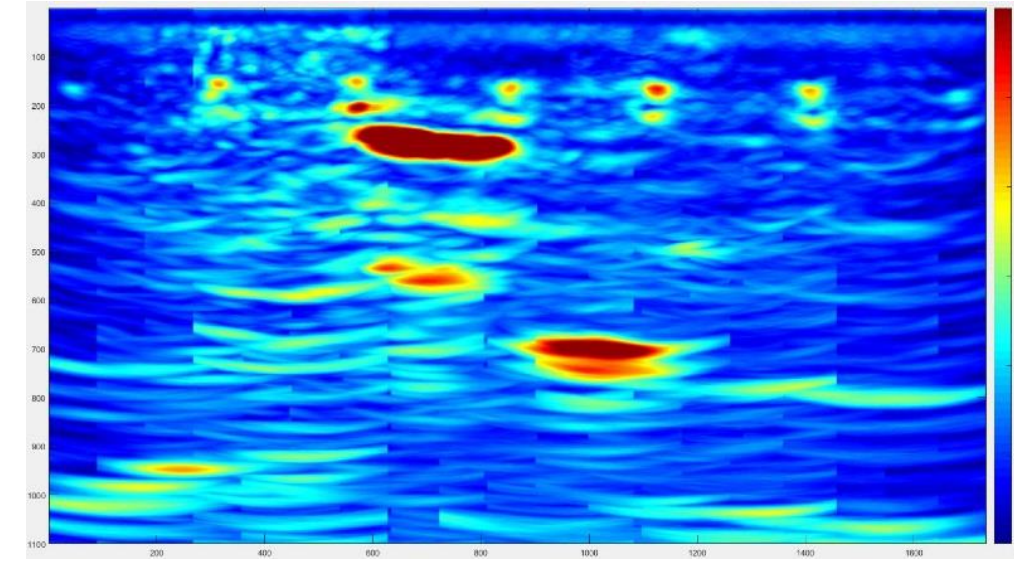

Two half-periods duration of the emitted signal

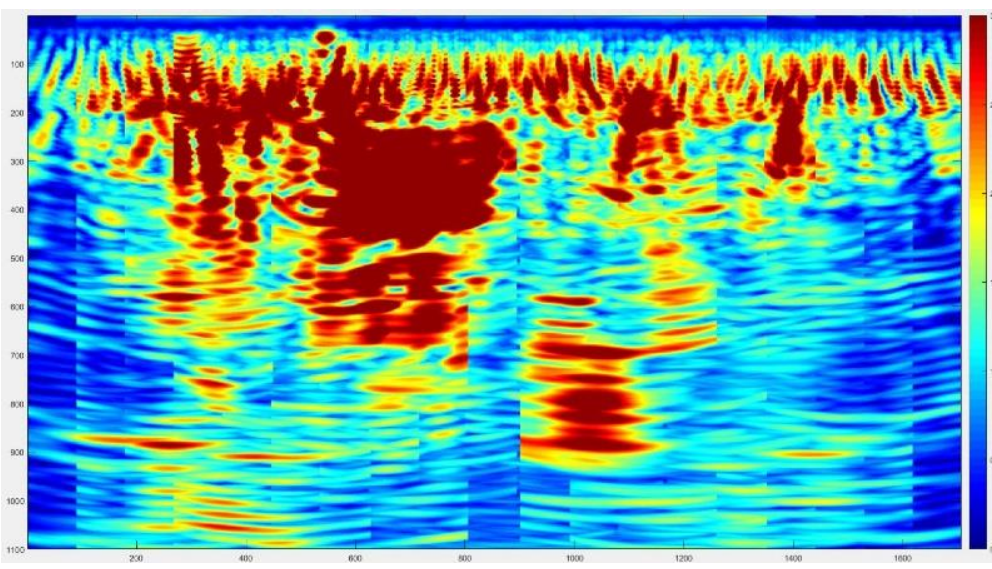

Sixteen half-periods duration of the emitted signal

Panoramic B-scan, row 9. 


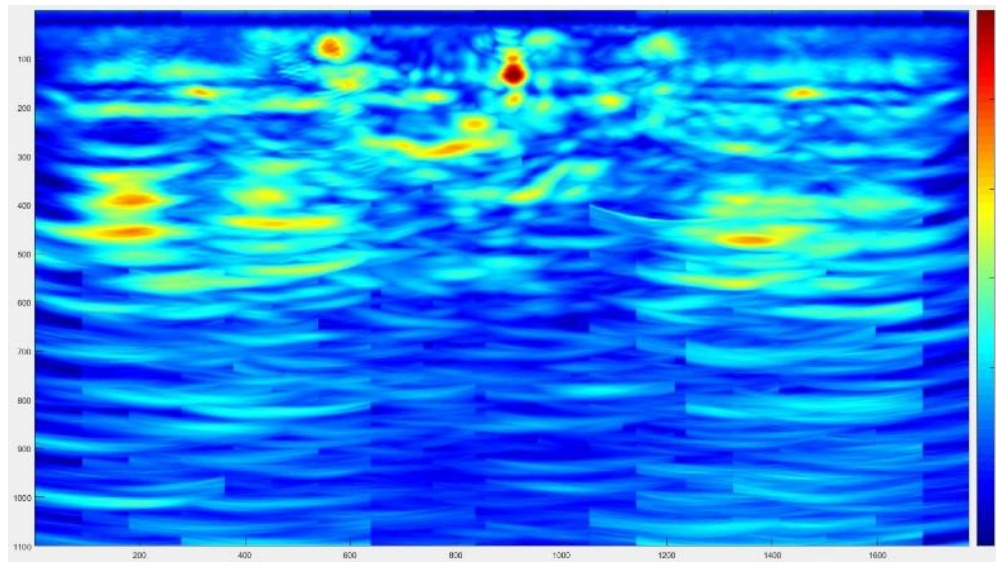

One half-period duration of the emitted signal

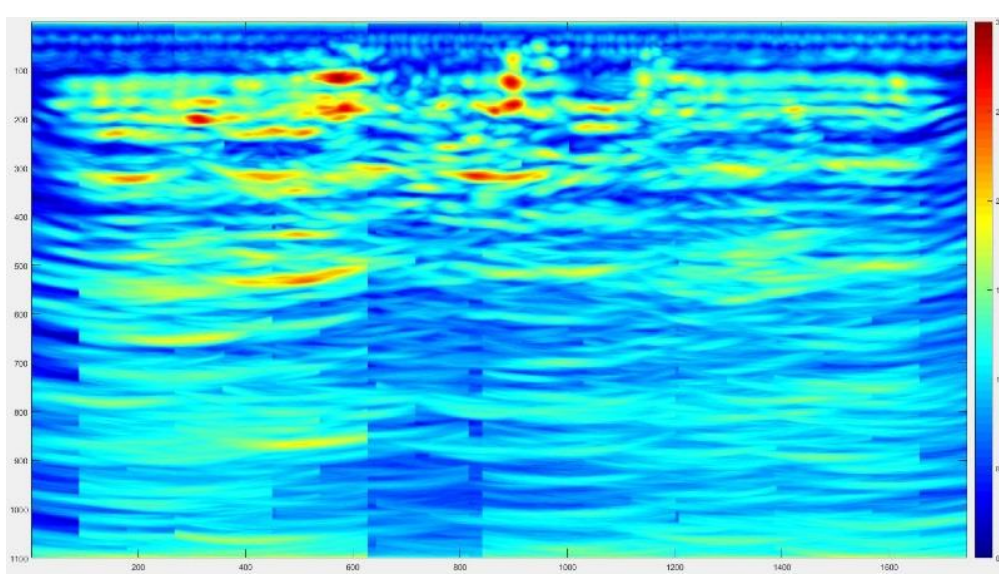

Four half-periods duration of the emitted signal

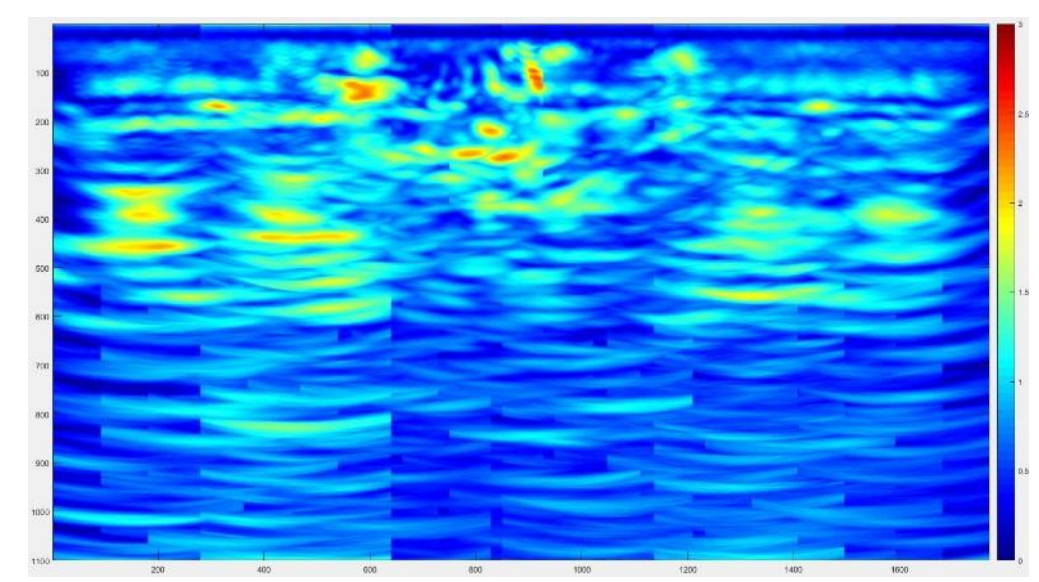

Two half-periods duration of the emitted signal

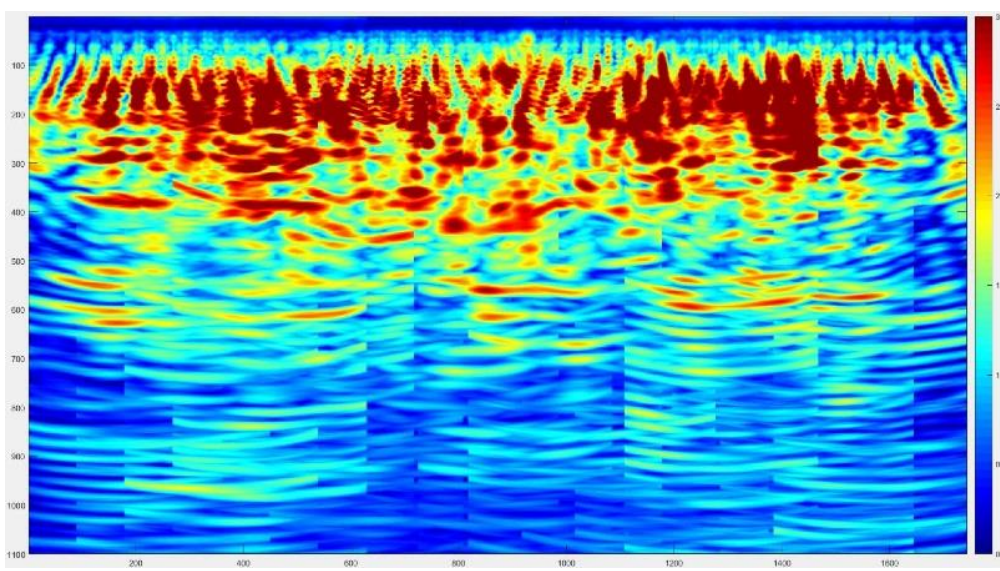

Sixteen half-periods duration of the emitted signal

Panoramic B-scan, row 10. 


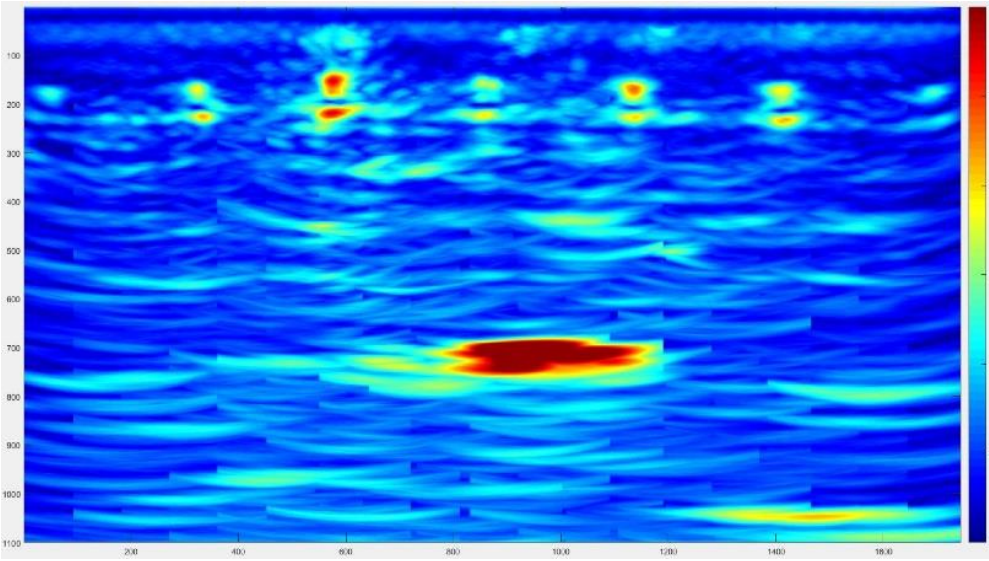

One half-period duration of the emitted signal

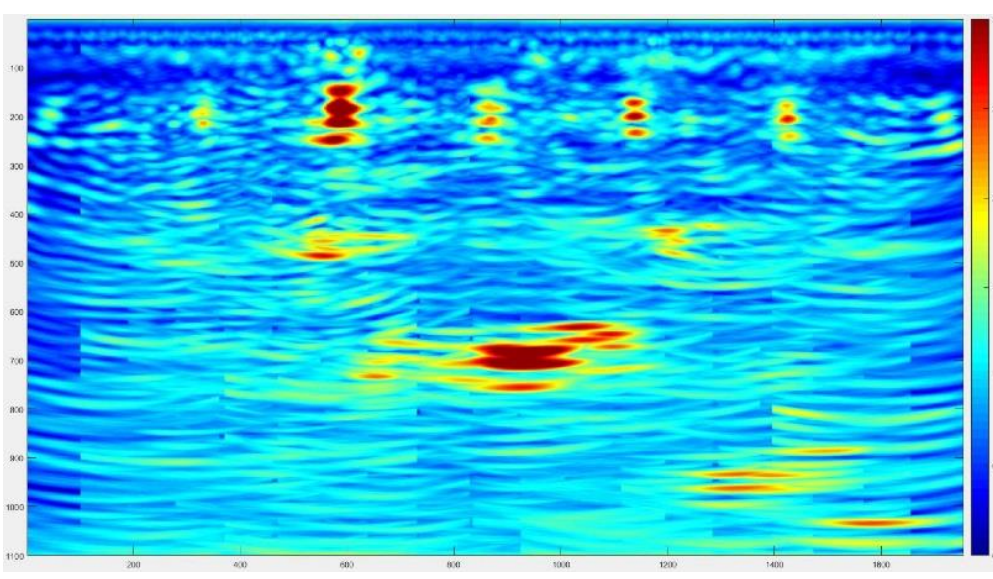

Four half-periods duration of the emitted signal

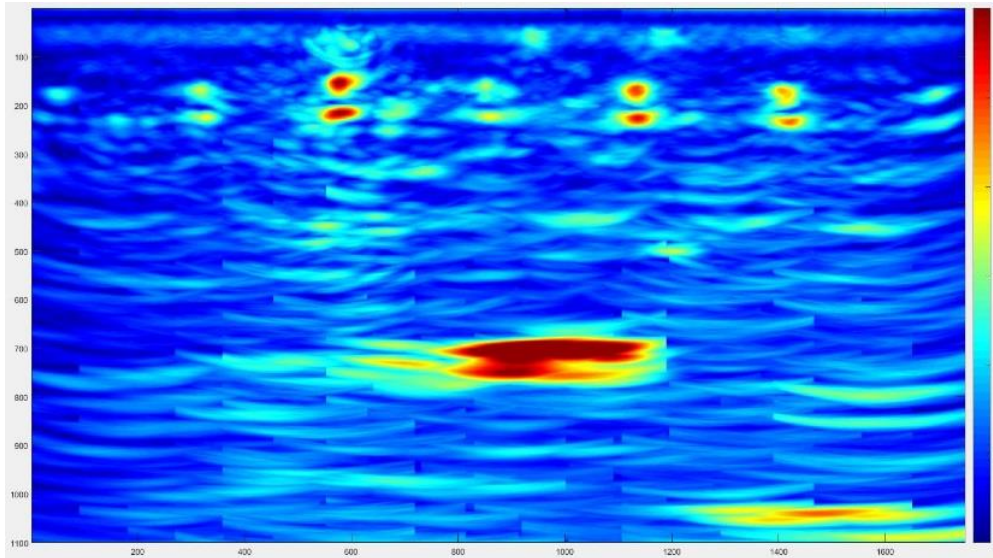

Two half-periods duration of the emitted signal

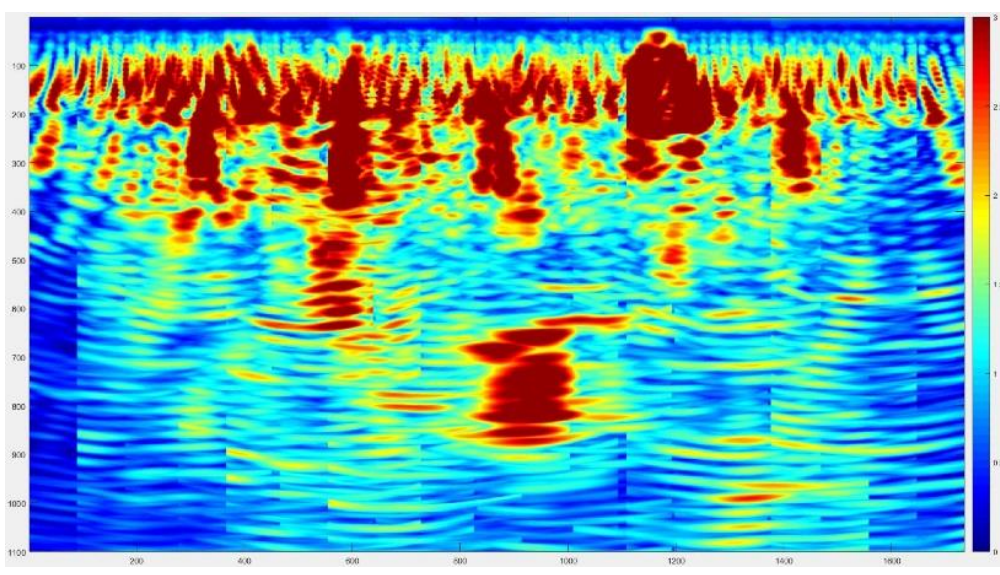

Sixteen half-periods duration of the emitted signal

Panoramic B-scan, row 11. 


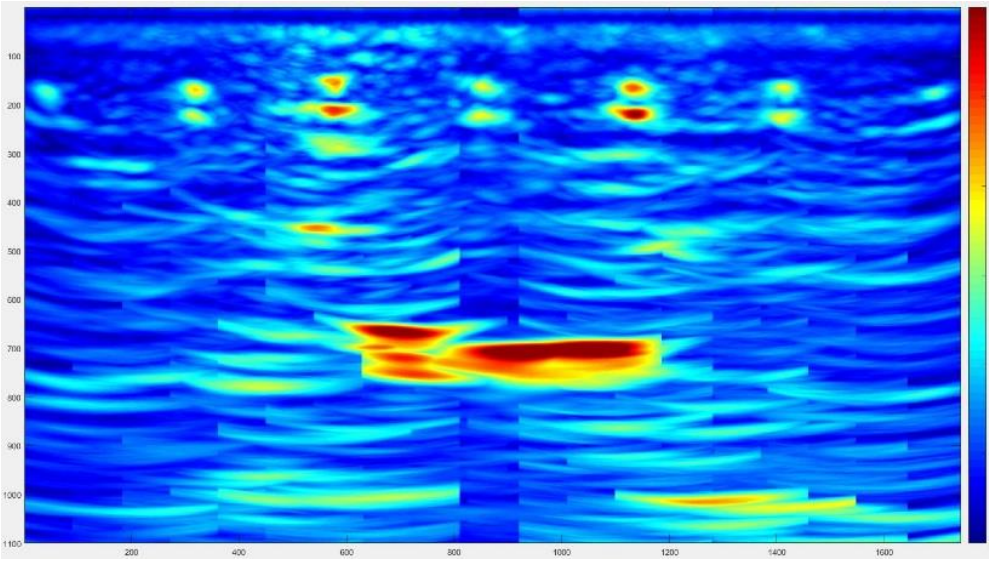

One half-period duration of the emitted signal

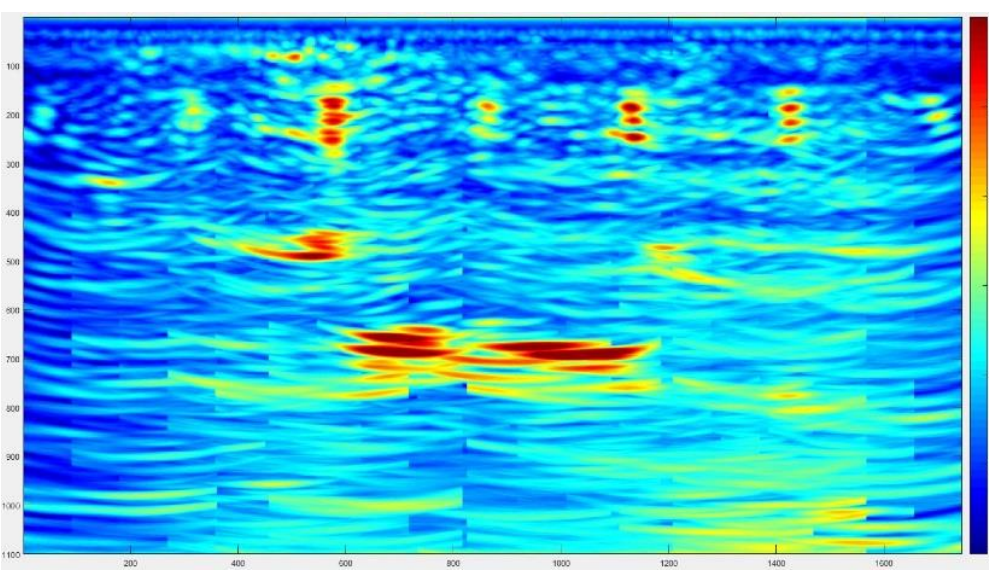

Four half-periods duration of the emitted signal

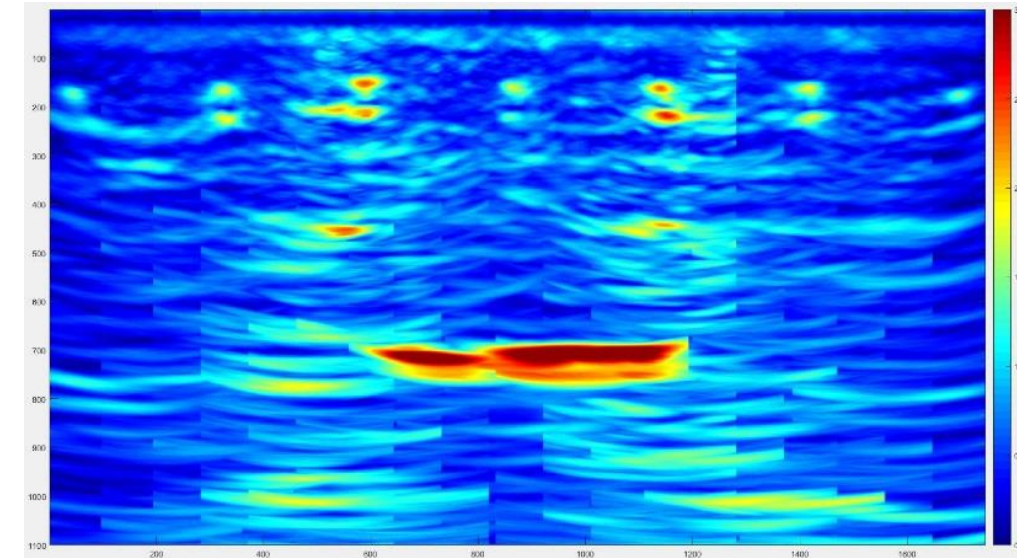

Two half-periods duration of the emitted signal

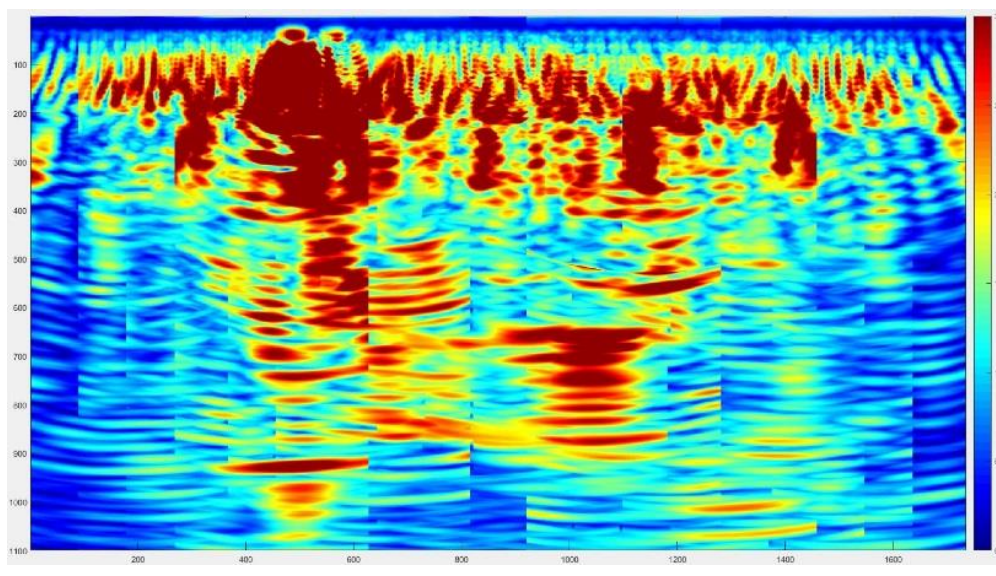

Sixteen half-periods duration of the emitted signal

Panoramic B-scan, row 12. 


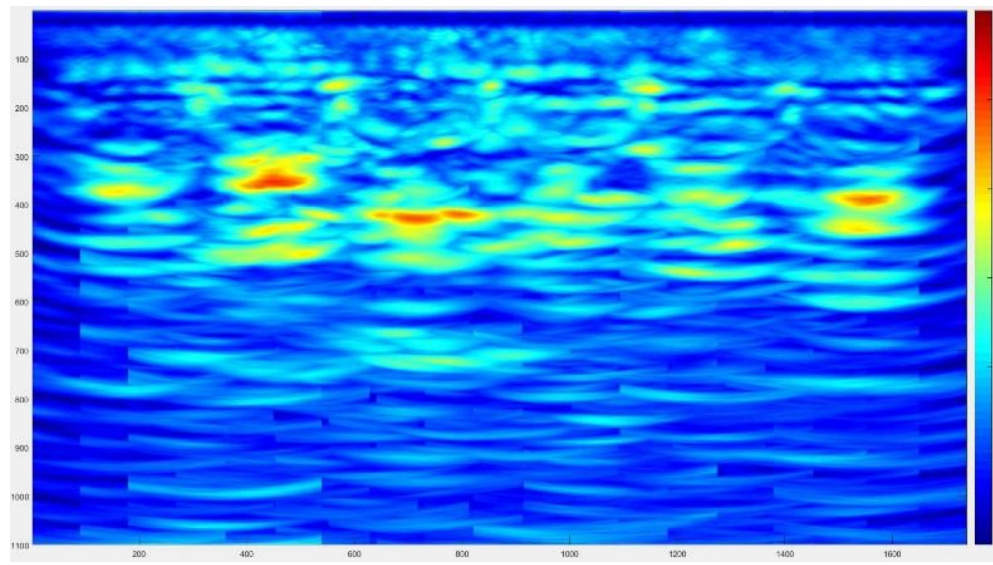

One half-period duration of the emitted signal

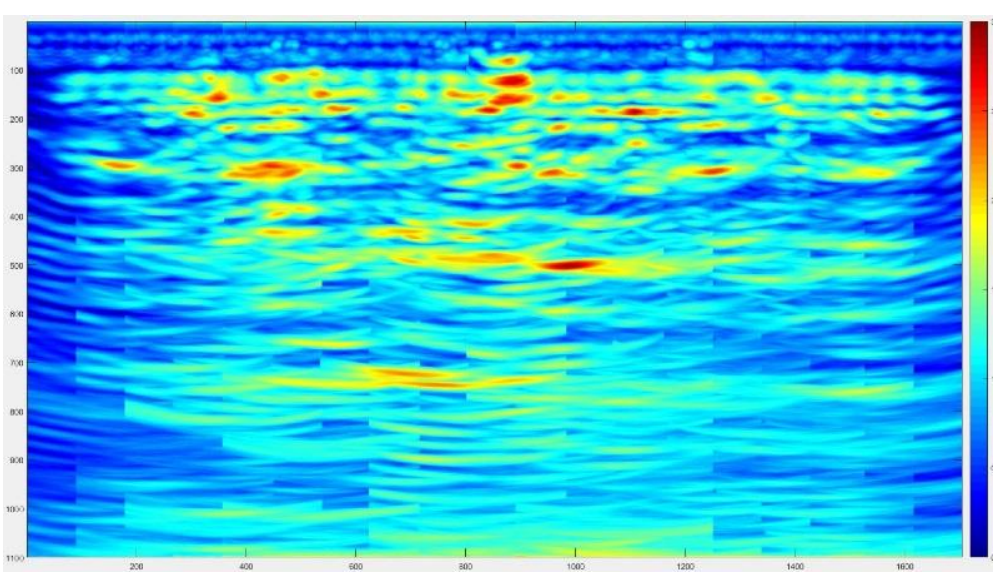

Four half-periods duration of the emitted signal

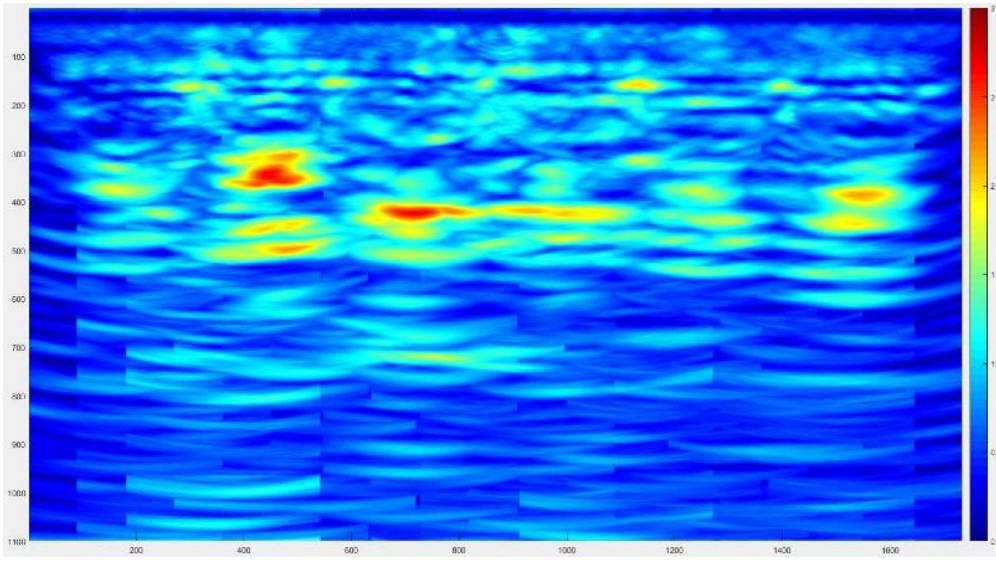

Two half-periods duration of the emitted signal

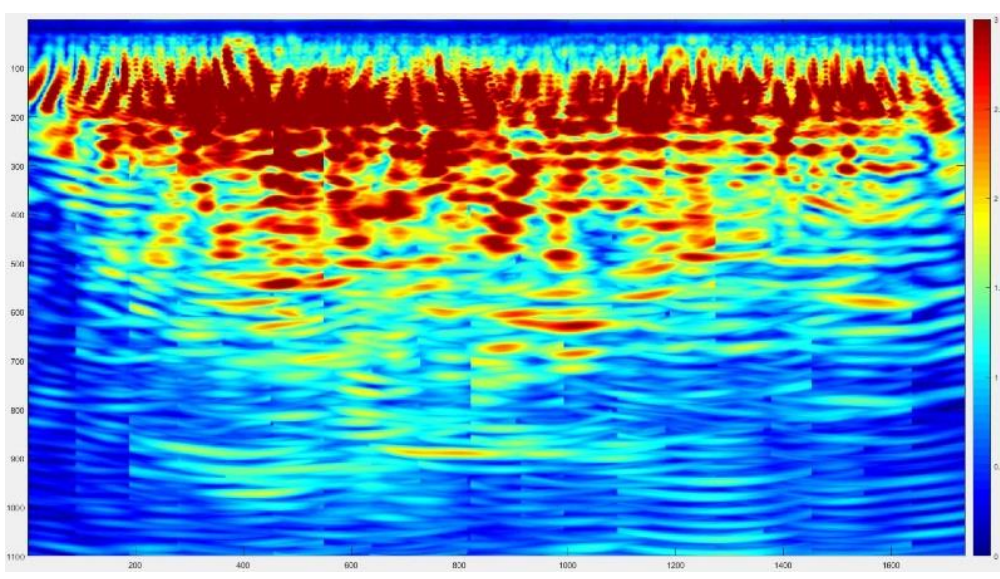

Sixteen half-periods duration of the emitted signal

Panoramic B-scan, row 13. 


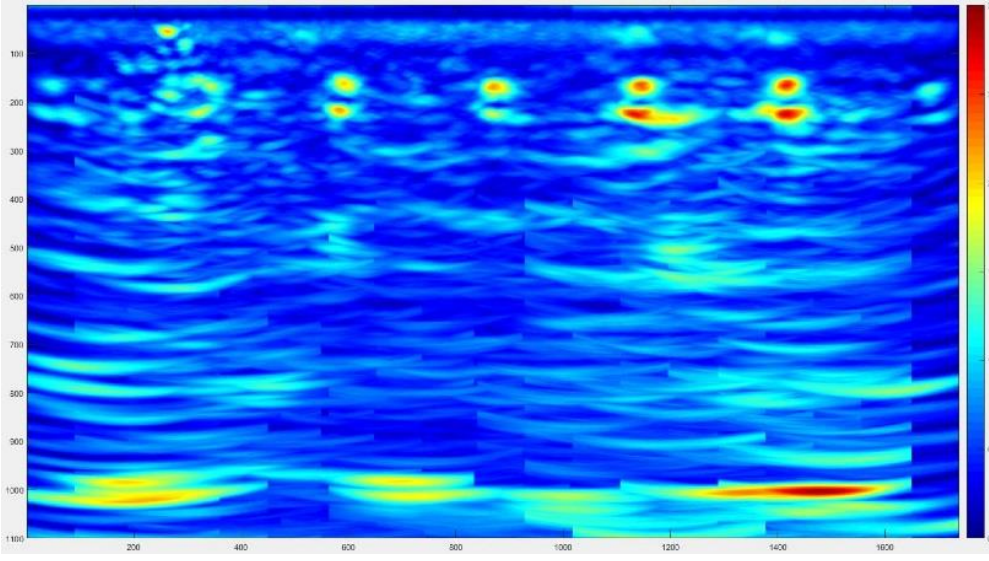

One half-period duration of the emitted signal

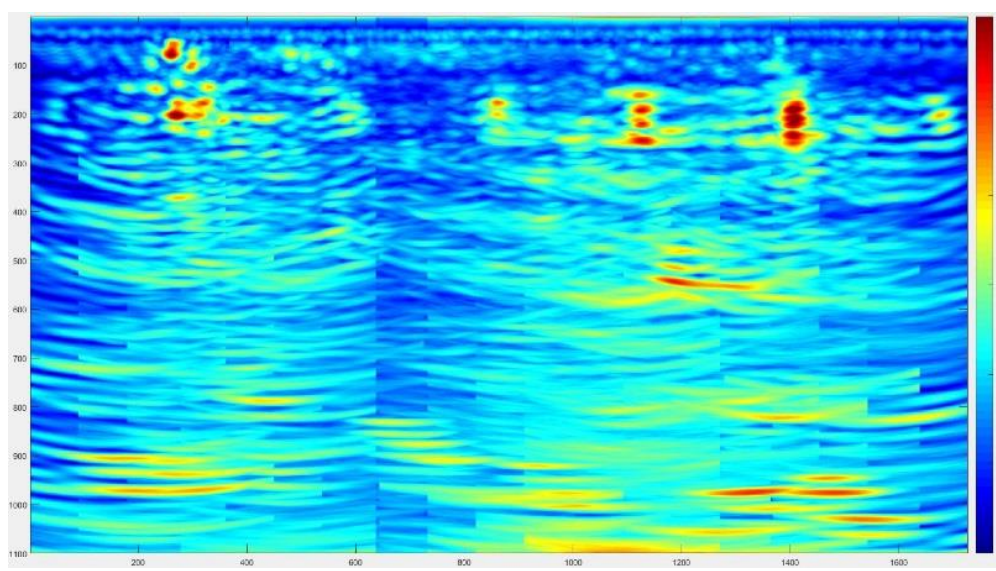

Four half-periods duration of the emitted signal

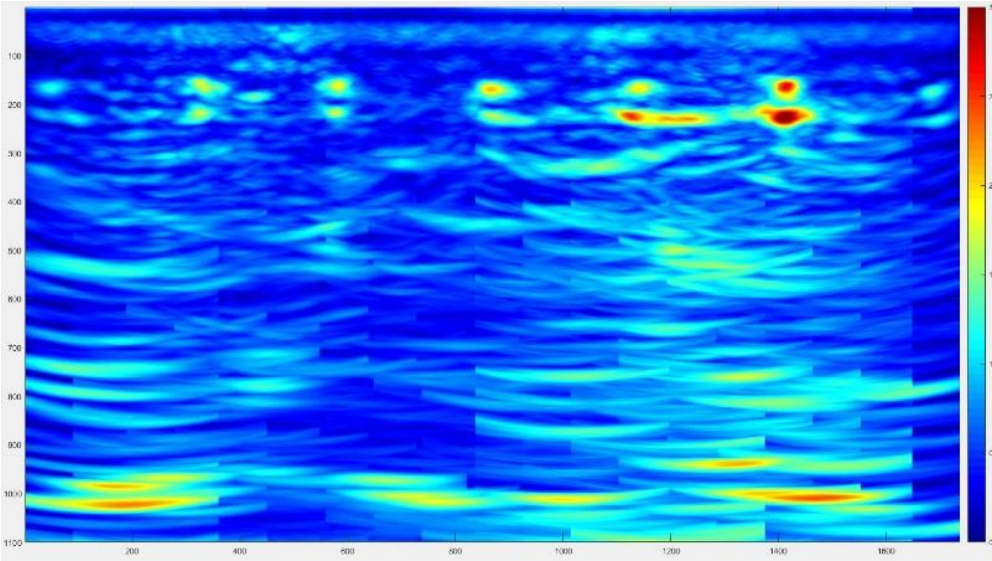

Two half-periods duration of the emitted signal

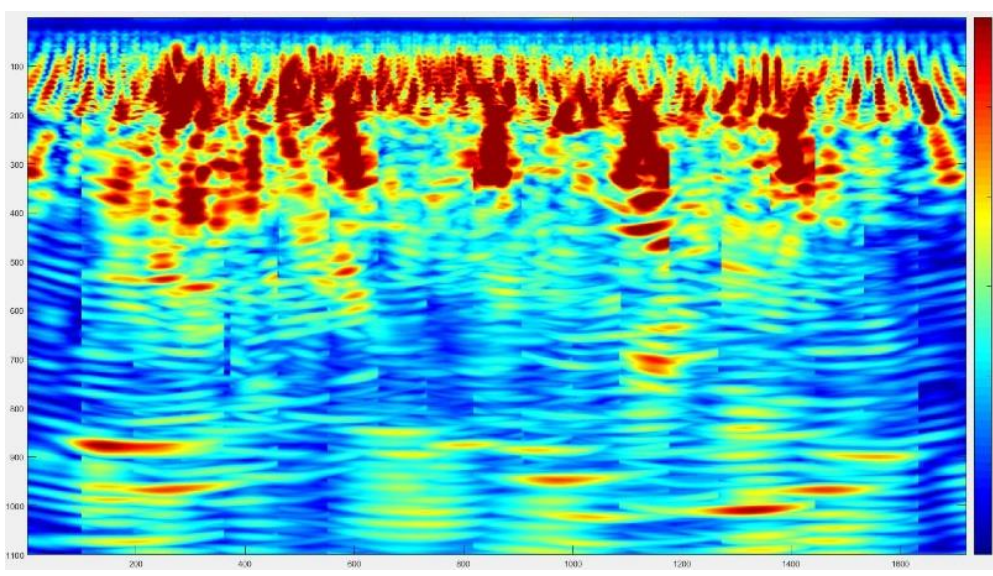

Sixteen half-periods duration of the emitted signal

Panoramic B-scan, row 14. 


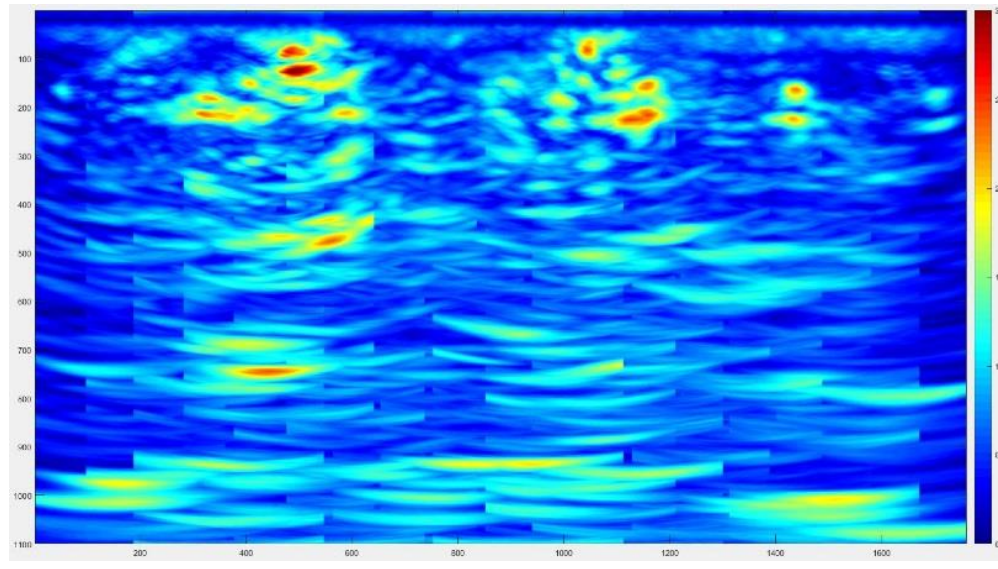

One half-period duration of the emitted signal

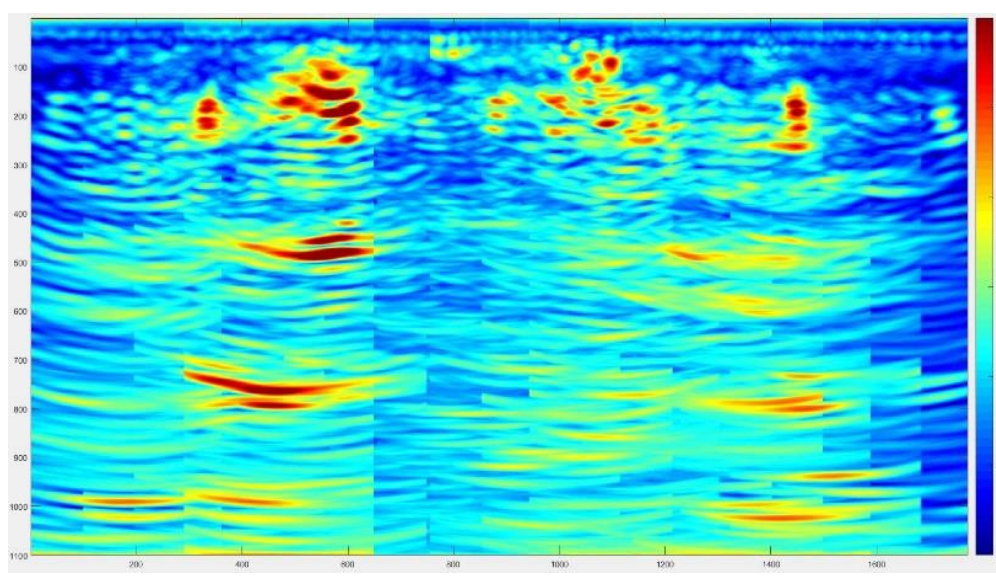

Four half-periods duration of the emitted signal

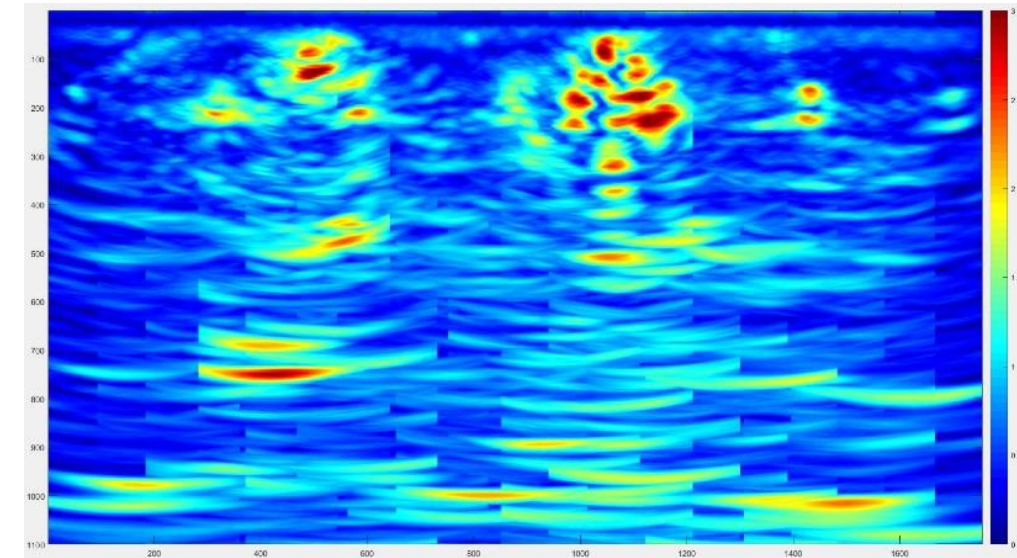

Two half-periods duration of the emitted signal

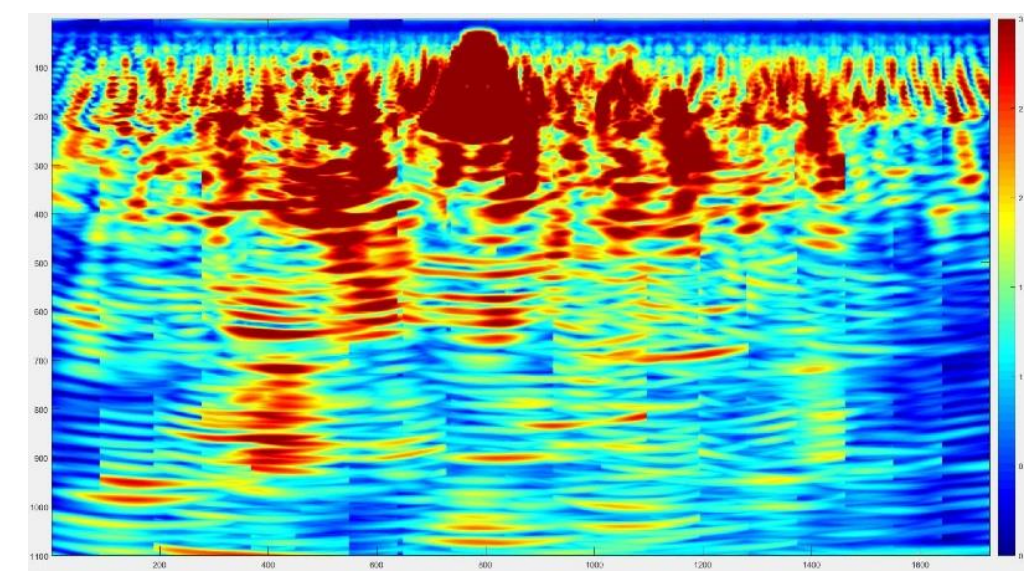

Sixteen half-periods duration of the emitted signal

Panoramic B-scan, row 15. 


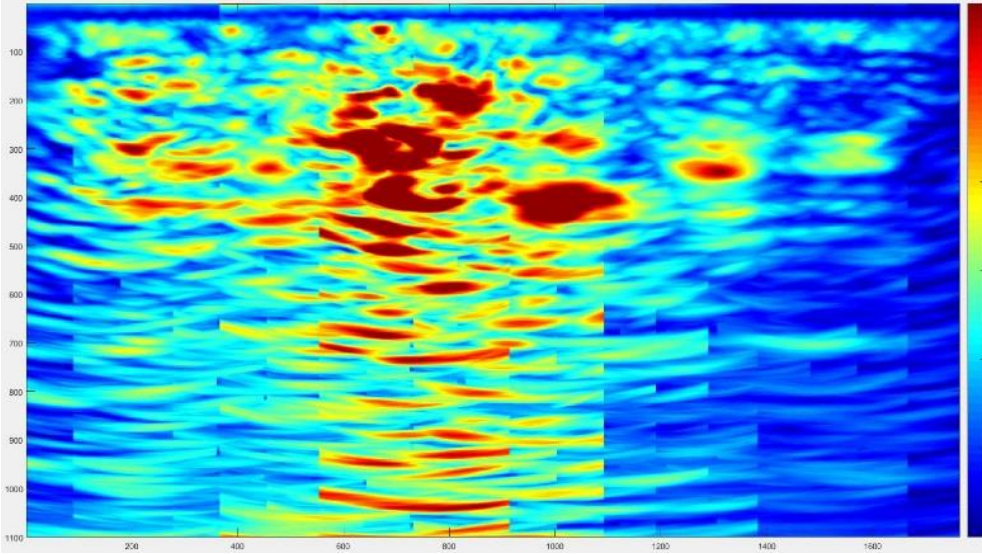

One half-period duration of the emitted signal

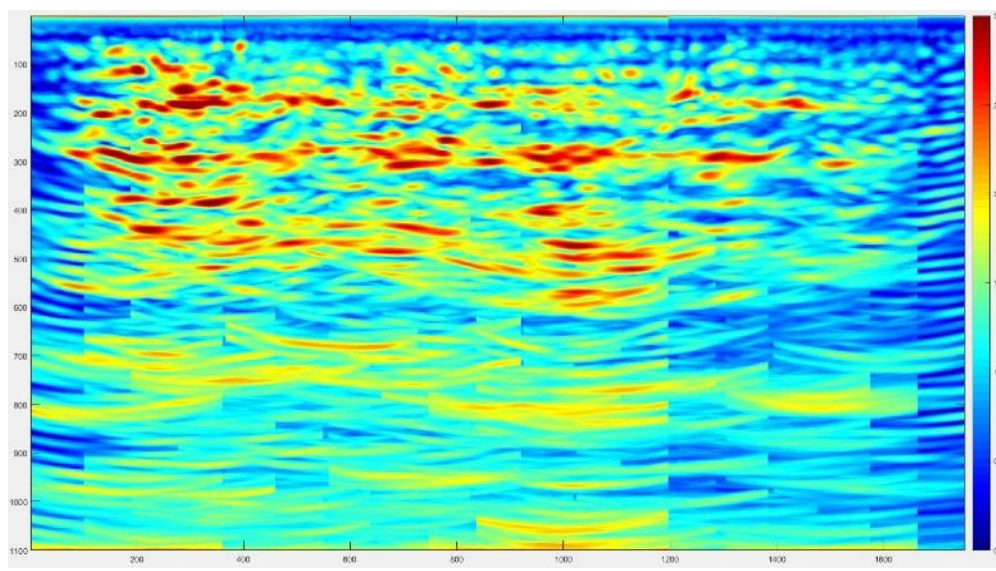

Four half-periods duration of the emitted signal

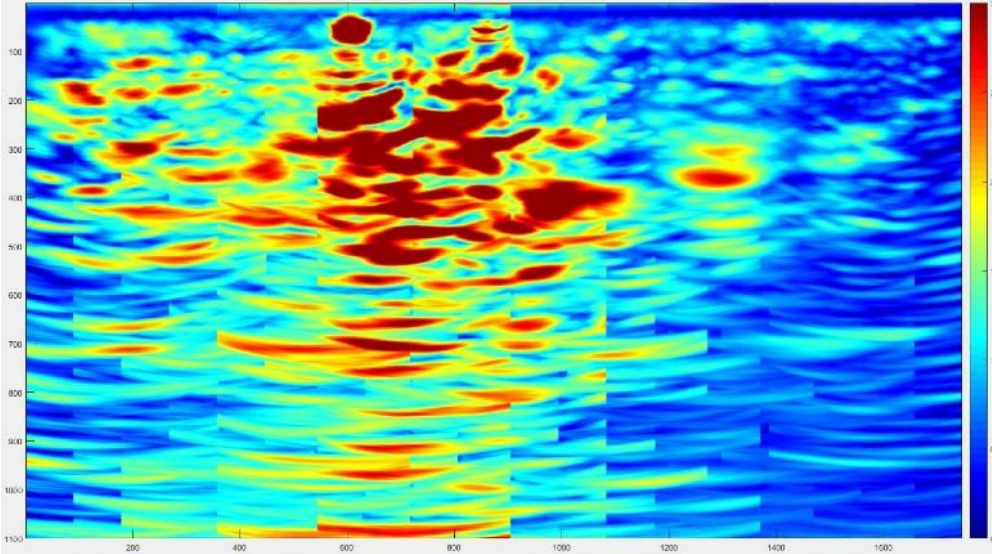

Two half-periods duration of the emitted signal

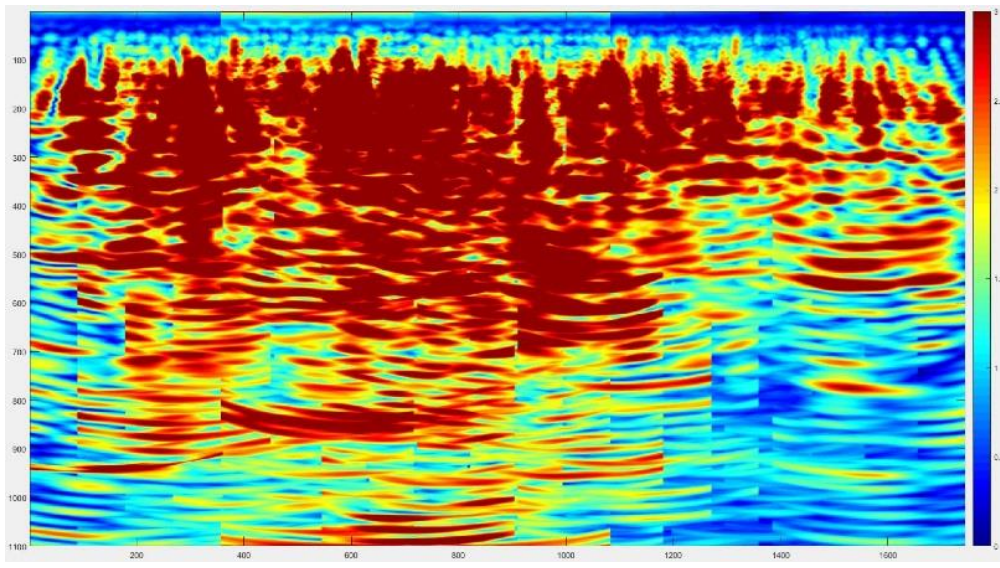

Sixteen half-periods duration of the emitted signal

Panoramic B-scan, row 16. 


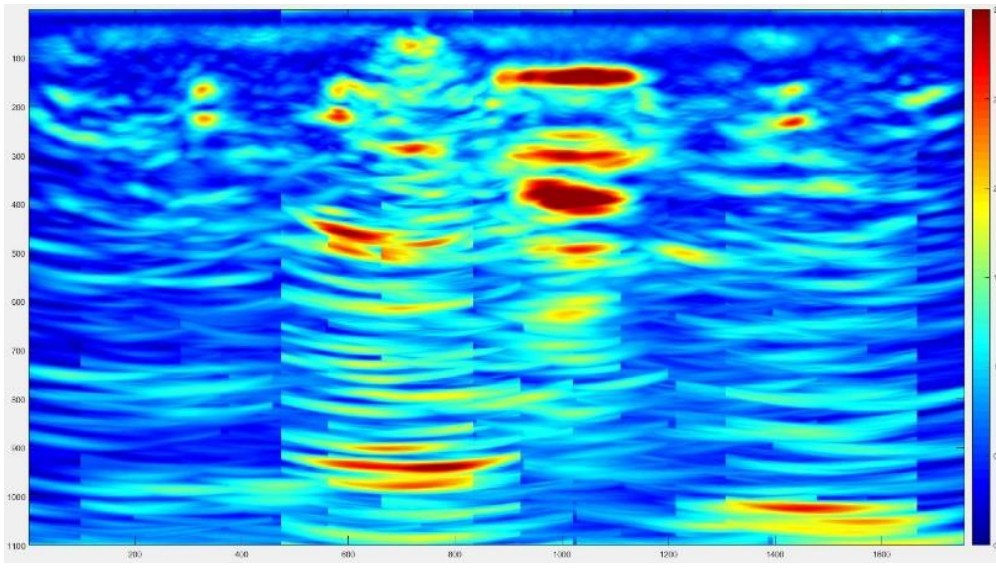

One half-period duration of the emitted signal

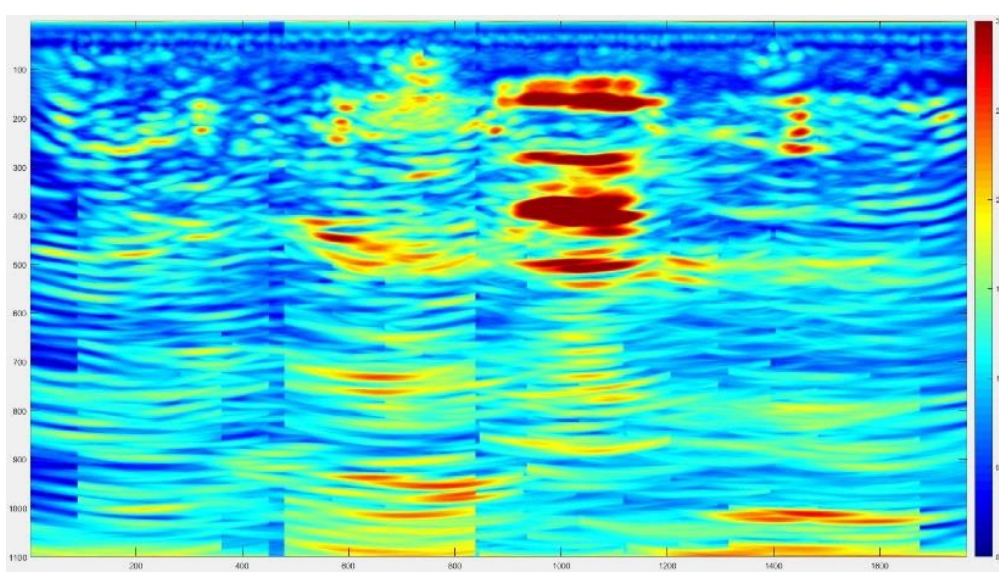

Four half-periods duration of the emitted signal

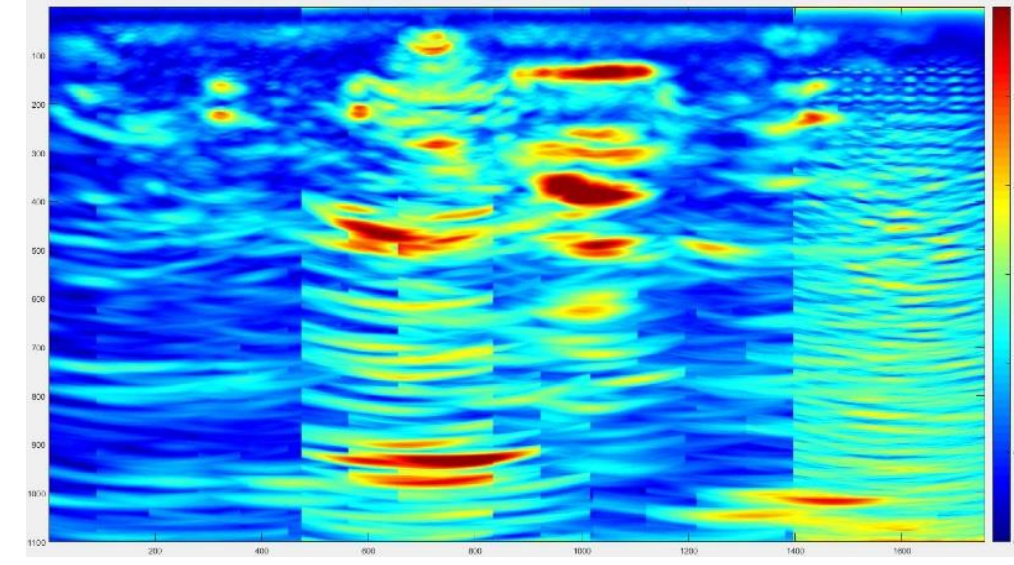

Two half-periods duration of the emitted signal

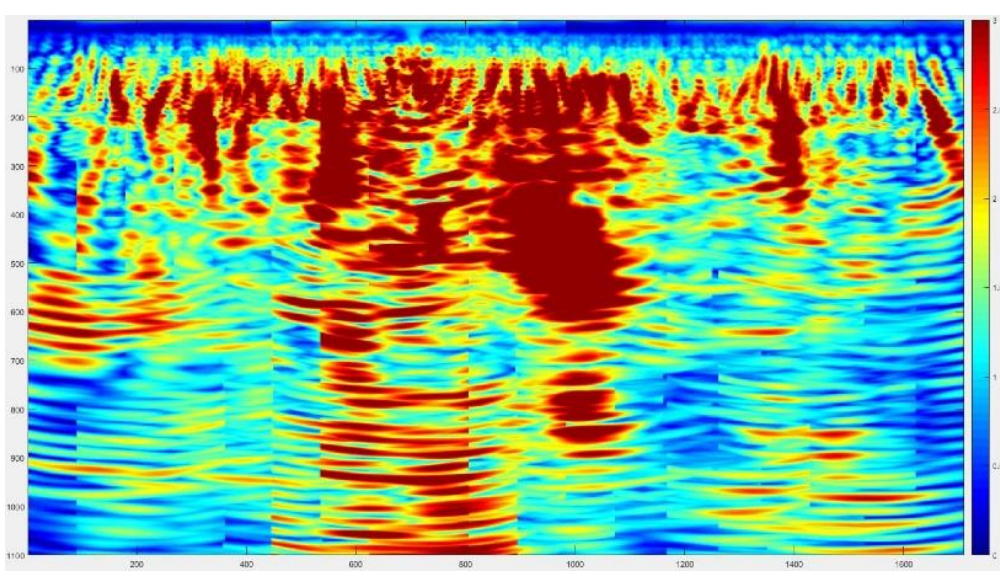

Sixteen half-periods duration of the emitted signal

Panoramic B-scan, row 17. 


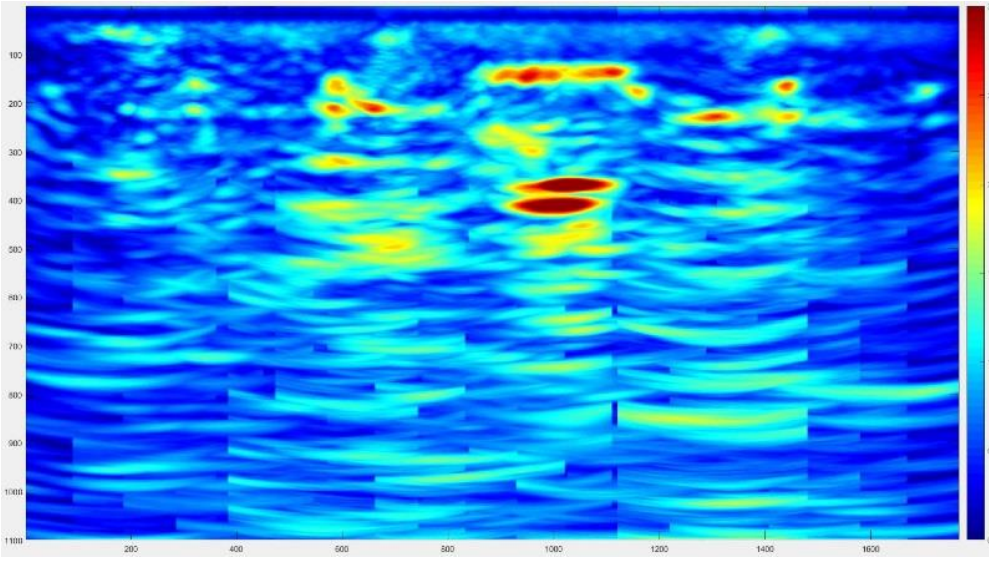

One half-period duration of the emitted signal

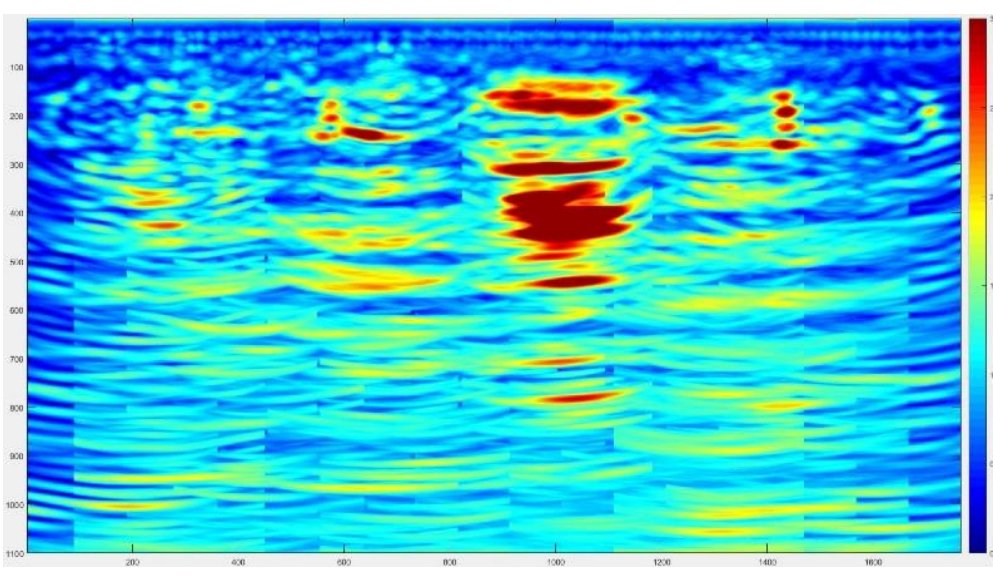

Four half-periods duration of the emitted signal

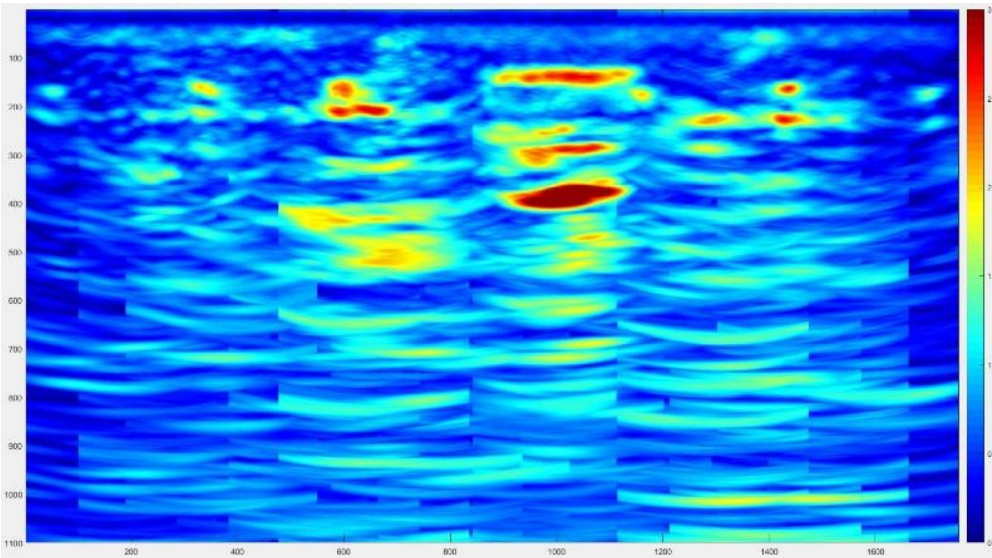

Two half-periods duration of the emitted signal

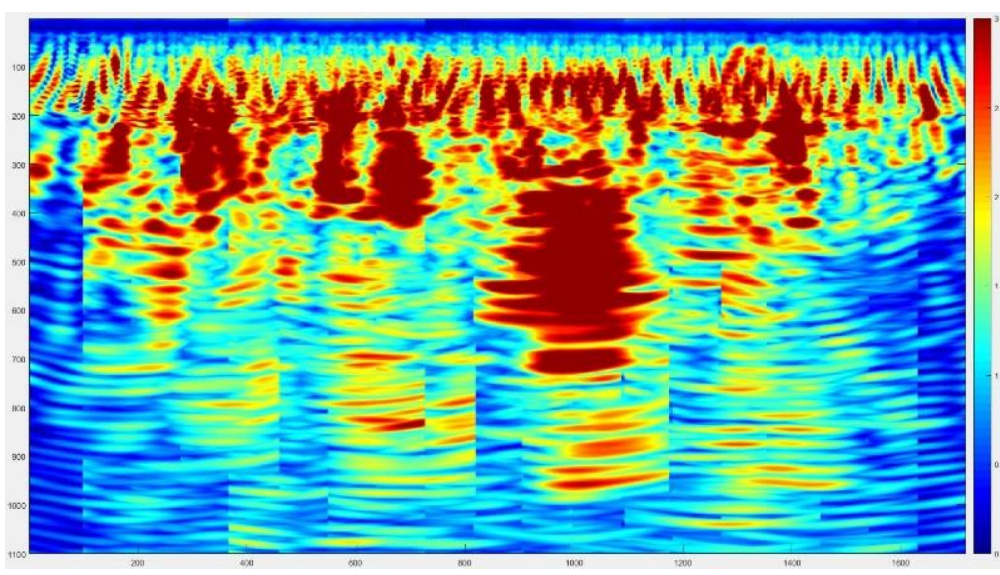

Sixteen half-periods duration of the emitted signal

Panoramic B-scan, row 18. 


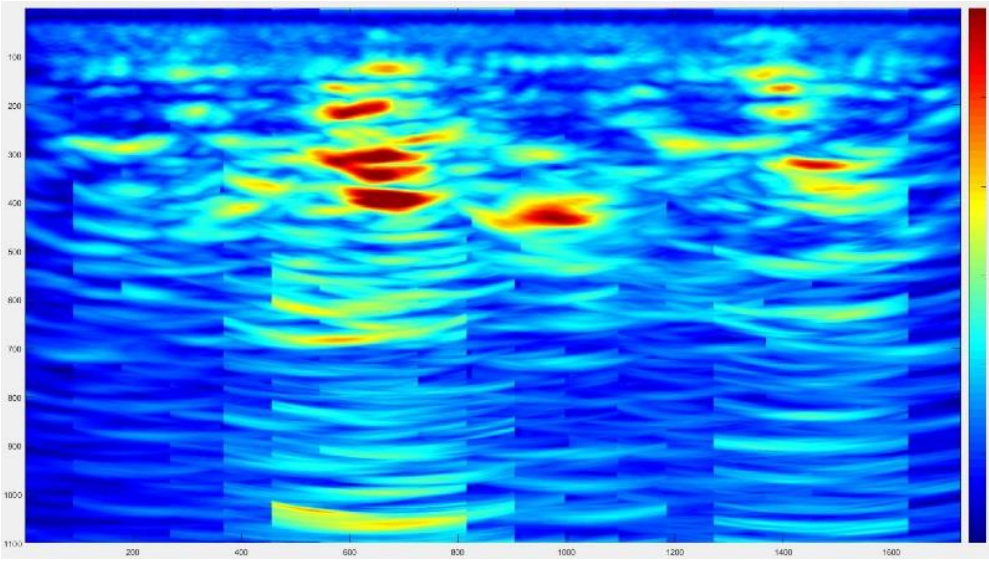

One half-period duration of the emitted signal

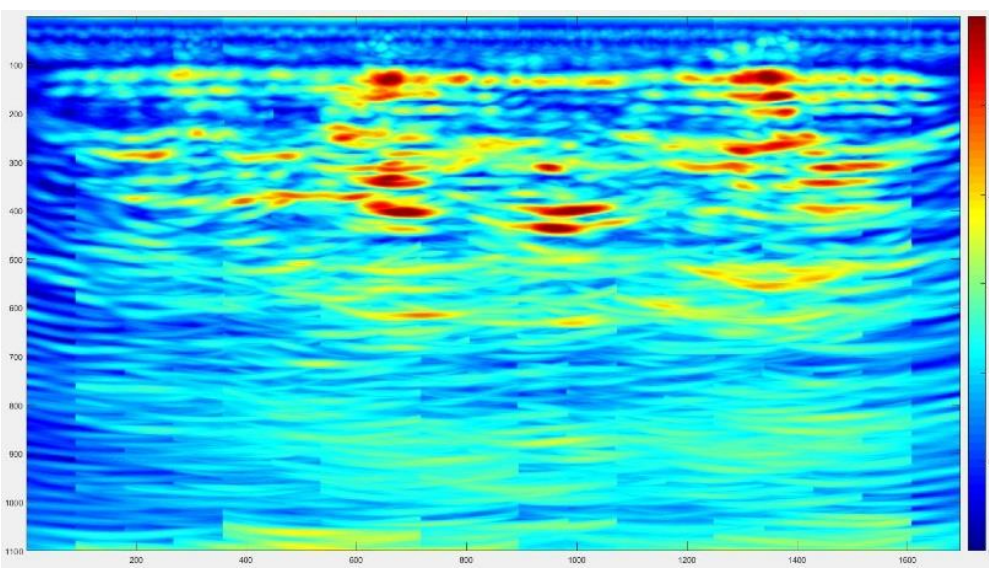

Four half-periods duration of the emitted signal

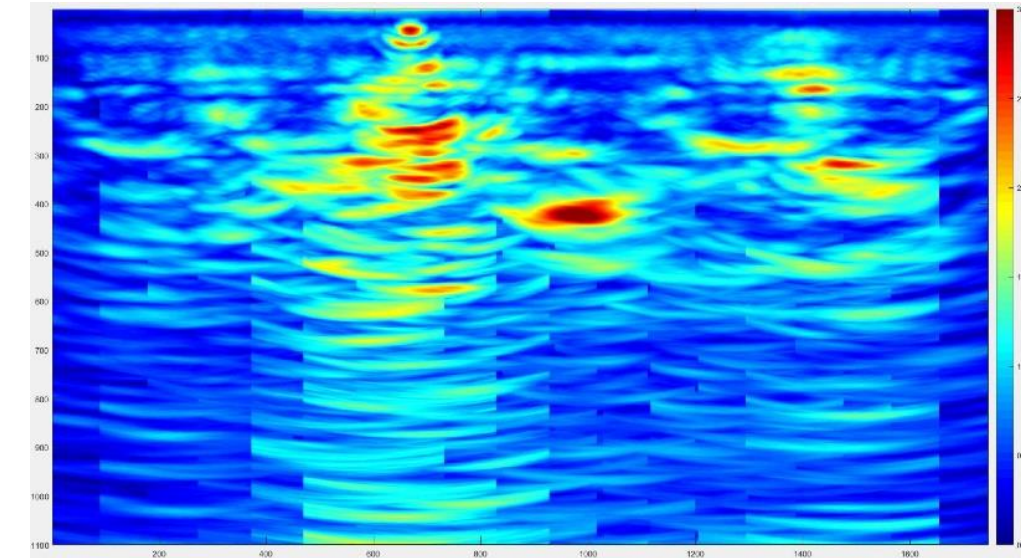

Two half-periods duration of the emitted signal

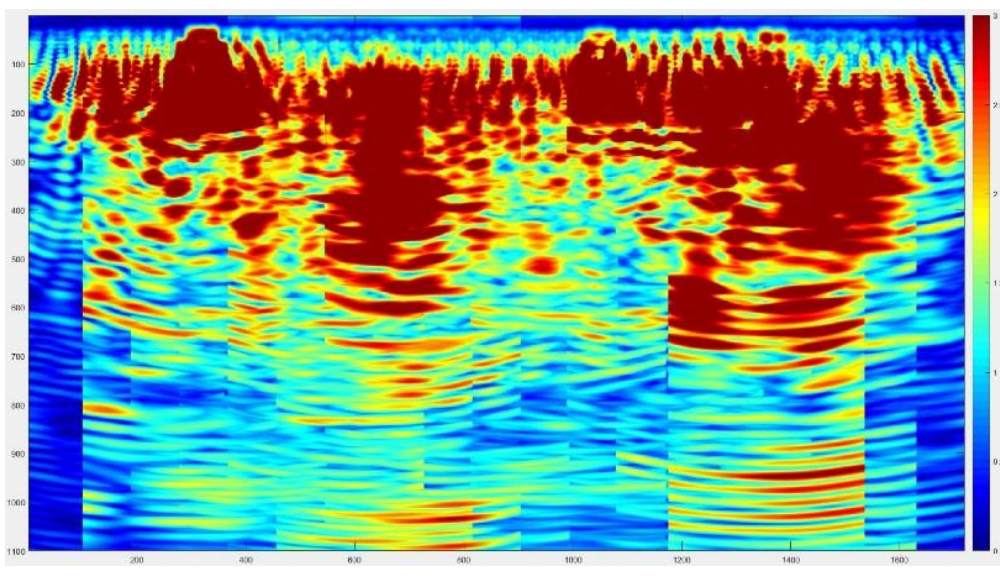

Sixteen half-periods duration of the emitted signal

Panoramic B-scan, row 19. 
